\title{
DEL PEZZO SURFACES AS SPRINGER FIBRES FOR EXCEPTIONAL GROUPS
}

I. GROJNOWSKI AND N. I. SHEPHERD-BARRON

\section{Introduction}

Since the discovery by Cayley and Salmon of the 27 lines on a cubic surface and their configuration and that by Killing and Cartan of the exceptional simple Lie algebra of type $E_{6}$ it has been clear that del Pezzo surfaces and exceptional simple algebraic groups have attached to them the same combinatorial objects. That is, there are well known constructions

(del Pezzo surfaces) $\rightarrow$ (exceptional root data) $\leftrightarrow$ (exceptional simple groups).

One of the results of this paper is a direct geometrical construction, for $5 \leq l \leq$ 8 , of the del Pezzo surfaces $S$ of degree $d=9-l$ from the split simply connected exceptional simple ${ }^{1}$ group $G$ of type $E_{l}$, in the presence of the universal elliptic curve $\mathcal{E}$. Direct, that is, in the sense that it does not involve root data.

The del Pezzo surfaces arise out of the group as follows. Let $P$ denote the weight lattice of $G$ and $B$ a Borel subgroup. Suppose that $\widetilde{\mathcal{G}}_{\mathcal{E}}^{s s}$ is the stack that classifies semi-stable $G$-bundles over elliptic curves together with a reduction of degree 0 of the structure group to $B$. (The existence of such a reduction is a characterization of semi-stability.) Then there is a partial relative compactification of $\widetilde{\mathcal{G}}_{\mathcal{E}}^{s s}$ in which the total space $\mathcal{D}_{1}^{-}$of a certain family $\mathcal{D}_{1}^{-} \rightarrow \mathcal{Y}=\operatorname{Hom}(P, \mathcal{E})$ of these del Pezzo surfaces appears as a boundary divisor. We shall describe this in more detail later in this introduction. (The del Pezzo surfaces in the family $\mathcal{D}_{1}^{-} \rightarrow \mathcal{Y}$ are in fact marked; that is, a copy of $\mathcal{E}$ is embedded in them as an anticanonical curve and their Néron-Severi groups are rigidified. The family is nearly, but not quite, universal; the fine moduli space is a non-separated union of copies of $\mathcal{Y}$, and $\mathcal{Y}$ is then its maximal separated quotient.)

However, we have no suggestion for a similarly direct construction of the groups from the del Pezzo surfaces that would bypass consideration of the root data.

The title of this paper refers to our presentation of these results as an extension of the construction and theorems due to Brieskorn, Grothendieck, Slodowy and Springer (BGSS) and described in detail in [Sl] that, in good characteristics, reveal the deformations and simultaneous resolution of du Val singularities (= rational

\footnotetext{
${ }^{1}$ We write $E_{5}=D_{5}$ and regard $D_{5}$ as exceptional. We also refer to groups as "simple" rather than "almost simple".
} 
double points $=$ simple singularities $=$ Kleinian singularities) of type $A, D$ or $E$ inside the corresponding split simply connected simple algebraic group. In turn, this provides a characterization of du Val singularities. Of course, there are many others. One, that we shall use, is that a surface singularity is du Val if and only if its monodromy group, acting on the vanishing cohomology, is the relevant Weyl group.

Our results lead, as a by-product, to an identification of the unipotent singularity of $E_{8}$ in bad characteristics (that is, 2,3 and 5). This is done in section 8. The proof depends upon specializing $\mathcal{E}$ to a uniformizable elliptic curve $E$ over a complete algebraically closed field of characteristic $p$. The picture is that the curve $E$ acts as a vector to carry some of the geometry of the group into a del Pezzo surface $S$ with a singularity isomorphic to the unipotent singularity, and we can then exploit the fact that the defining equations of such a surface are so simple that classification of the unipotent singularities becomes very easy and quick. Moreover, this leads to an embedding of the group-theoretical description of the deformations and resolutions of du Val singularities into their description in terms of del Pezzo surfaces, as expounded in [SSS].

This is, perhaps, some slight evidence supporting a view that our results tell us something about the group, and that further information about the group is to be found in higher codimension in suitable relative compactifications of $\widetilde{\mathcal{G}}_{\mathcal{E}}^{s s}$.

In the rest of this introduction we give a more detailed survey of our results.

From the viewpoint of the geometry of algebraic surfaces and their degenerations the extension referred to above consists of showing that simultaneous log resolutions, which are defined in the next paragraph, of simply elliptic singularities of degrees $d=1,2,3$ or 4 , which were first observed in the context of type II degenerations of K3 surfaces, although inspired by work of Brieskorn [B] and Looijenga [L], also have realizations in terms of simple algebraic groups. Here, only exceptional groups of type $E_{9-d}$ can, and do, occur.

We define a del Pezzo surface to be a surface $S$ whose anti-canonical divisor class is ample and that has at worst du Val singularities; a weak del Pezzo surface is smooth and its anti-canonical class is ample modulo finitely many $(-2)$-curves. That is, a weak del Pezzo surface is the minimal resolution of a del Pezzo surface and a del Pezzo surface is the anticanonical model of a weak del Pezzo surface.

Definition 1.1 Given a flat family $X \rightarrow S$ of normal Gorenstein surfaces, a simultaneous log resolution of $X \rightarrow S$ is a commutative diagram

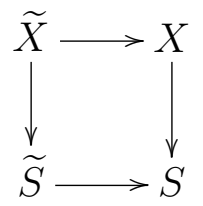

where $\widetilde{S} \rightarrow S$ is proper, dominant and generically finite, $\widetilde{X} \rightarrow X \times_{S} \widetilde{S}$ is proper and birational, $\widetilde{X}$ is smooth, $\widetilde{X} \rightarrow \widetilde{S}$ is flat and semi-stable, in the sense that 
each geometric fibre is a reduced union of smooth surfaces with normal crossings, and the relative canonical class $K_{\widetilde{X} / \widetilde{S}}$ is equivalent to the pullback of $K_{X / S}$.

It is well known in the context of the birational geometry of degenerating families of K3 surfaces that a versal deformation $X \rightarrow S$ of a simply elliptic singularity of degree at most 4 possesses a simultaneous $\log$ resolution $\widetilde{X} \rightarrow \widetilde{S}$ with the following properties.

(1) $\widetilde{X} \rightarrow \widetilde{S}$ is of type $I I$, in the sense that, for each fibre $X_{s}$ with an elliptic singularity, its inverse image $\tilde{X}_{\tilde{s}}$ in $\tilde{X}$ has two components, say $\widetilde{X}_{\tilde{s}}=D_{1}+D_{2}$. Moreover $D_{1}$ is the minimal resolution of $X_{s}, D_{2}$ is a weak del Pezzo surface, $D_{2}$ is contracted to a point under $\widetilde{X} \rightarrow X$ and $D_{1} \cap D_{2}$ is the elliptic curve that is the exceptional locus of $D_{1} \rightarrow X_{s}$.

(2) For each fibre $X_{s}$ that has at most du Val singularities, its inverse image $\widetilde{X}_{\tilde{s}}$ in $\tilde{X}$ is the minimal resolution of $X_{s}$.

(3) the base change $\widetilde{S} \rightarrow S$ is the composite of a ramified Galois covering of the base $S$ whose Galois group is the corresponding finite Weyl group and a blow-up along the simply elliptic locus in $S$.

On the other hand, Helmke and Slodowy [HS1] and [HS3] have given a brief indication of a proof that miniversal deformations of simply elliptic singularities of degree $d$ can be realized inside $\mathcal{G}$ when $G=E_{9-d}$.

Recall from [HS2], Theorems 5.6 and 5.12, that a regular bundle on an elliptic curve $E$ over a field is one whose automorphism group has minimal dimension, namely $l$, while for a subregular bundle the automorphism group has the next smallest dimension, $l+2$. Up to translation by points of $E$ there is a unique unstable regular bundle $\eta$ and a unique unstable subregular bundle $\xi$; in a miniversal deformation of $\xi$ the unstable locus is, up to this translation, a surface $S$ and all points of $S-\{\xi\}$ correspond to bundles isomorphic to $\eta$. Note that in order to construct these bundles it is necessary (and sufficient) for the group to be split and the curve $E$ to have a point; it cannot be replaced by an arbitrary curve of genus 1. Then both $\xi$ and $\eta$ are constructed as bundles on $\mathcal{E}$.

Let's list the objects that appear in our main theorem. These are:

(1) the stack $\mathcal{G}=\mathcal{G}_{\mathcal{E}}$ of principal $G$-bundles over the universal elliptic curve $\mathcal{E}$ (defined over the moduli stack $\mathcal{M}_{E l l}$ of elliptic curves) and the open substack $\mathcal{G}^{\text {ss }}$ of semi-stable bundles;

(2) the stack $\widetilde{\mathcal{G}}^{s s}$ that classifies semi-stable $G$-bundles together with a reduction of the structure group to a Borel subgroup such that the associated torus bundle is of degree 0 (in the course of reaching our main result we shall show that $\widetilde{\mathcal{G}}^{\text {ss }}$ is proper, representable and generically finite, of degree equal to the order of the Weyl group $W$ of $G$, over $\mathcal{G}^{s s}$, and that, when we restrict attention to a uniformizable elliptic curve, this structure reproduces the BGSS picture);

(3) stacks $\widetilde{\mathcal{G}}$ and $\widetilde{\mathcal{G}}^{+}$(to be described in Section 5), a projective birational morphism $\widetilde{\mathcal{G}} \rightarrow \widetilde{\mathcal{G}}^{+}$and a morphism which is proper but not representable (although its fibres have finite automorphism groups) $\widetilde{\mathcal{G}}^{+} \rightarrow \mathcal{G}$ such that $\widetilde{\mathcal{G}} \rightarrow \mathcal{G}$ and $\widetilde{\mathcal{G}}^{+} \rightarrow \mathcal{G}$ 
are relative compactifications of $\widetilde{\mathcal{G}}^{s s} \rightarrow \mathcal{G}^{s s}$

(4) the abelian variety $\mathcal{Y}=\operatorname{Hom}(P, \mathcal{E})$ over $\mathcal{M}_{\text {Ell }}$ and a certain ample $W$ linearized line bundle $\mathcal{L} \rightarrow \mathcal{Y}$, which gives a cone $\widehat{\mathcal{Y}}$ over $\mathcal{M}_{\text {Ell }}$ by contracting the zero section $0_{\mathcal{L}}$ of $\mathcal{L}$ such that the geometric quotient $[\widehat{\mathcal{Y}} / W]$ of $\widehat{\mathcal{Y}}$ by $W$, relative to $\mathcal{M}_{E l l}$, is, by [L], an affine space bundle of rank $l+1$ over $\mathcal{M}_{E l l}$;

(5) the unstable subregular bundle $\xi$ mentioned above, that is defined over $\mathcal{M}_{E l l}$ and is unique modulo translation by $\mathcal{E}$;

(6) the base $\mathcal{Z}$ of a deformation that is minimally versal, modulo the translation by $\mathcal{E}$ mentioned above, of $\xi$;

(7) the products $\widetilde{\mathcal{Z}}=\mathcal{Z} \times{ }_{\mathcal{G}} \widetilde{\mathcal{G}}$, and $\widetilde{\mathcal{Z}}^{+}=\mathcal{Z} \times{ }_{\mathcal{G}} \widetilde{\mathcal{G}}^{+}$and a birational map (not a morphism) $\widetilde{\mathcal{Z}}^{+}-\rightarrow \widetilde{\mathcal{Z}}^{-}$which is constructed as a succession of flops relative to $\mathcal{Z}$ such that the morphisms $\widetilde{\mathcal{Z}} \rightarrow \mathcal{Z}$ and $\widetilde{\mathcal{Z}}^{ \pm} \rightarrow \mathcal{Z}$ are representable.

Moreover, let ${ }^{0} \mathcal{G}$ be the open substack of $\mathcal{G}$ that is the complement of the locus of those unstable bundles $\Xi$ such that $\operatorname{dim} \operatorname{Aut}(\Xi)>l+4$. That is, the locus of unstable bundles that are "worse than subregular" has been deleted. In particular, $\mathcal{G}^{s s}$ is contained in ${ }^{0} \mathcal{G}$. Set ${ }^{0} \widetilde{\mathcal{G}}=\widetilde{\mathcal{G}} \times{ }_{\mathcal{G}}{ }^{0} \mathcal{G}$ and ${ }^{0} \widetilde{\mathcal{G}}^{+}=\widetilde{\mathcal{G}}^{+} \times{ }_{\mathcal{G}}{ }^{0} \mathcal{G}$, so that $\mathcal{Z}$ is, up to turning off $\operatorname{Pic}^{1}(\mathcal{E})$, a chart in ${ }^{0} \mathcal{G}$ and $\widetilde{\mathcal{Z}}$ is a chart in ${ }^{0} \widetilde{\mathcal{G}}$. Then the birational morphism $\widetilde{\mathcal{Z}} \rightarrow \widetilde{\mathcal{Z}}^{+}$and the birational maps $\widetilde{\mathcal{Z}}^{+}-\rightarrow \widetilde{\mathcal{Z}}^{-}$and $\widetilde{\mathcal{Z}}-\rightarrow \widetilde{\mathcal{Z}}^{-}$are all isomorphisms over $\mathcal{G}^{s s}$; that is, all birational modifications are made inside the boundary of ${ }^{0} \widetilde{\mathcal{G}}$ and the commutative square

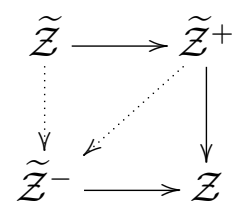

of rational maps and morphisms is a local description of a commutative square

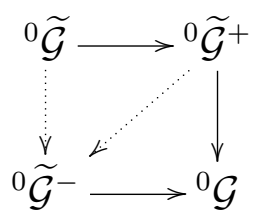

of rational maps and morphisms between stacks.

What is missing from this picture is a suitable stack $\widetilde{\mathcal{G}}^{-}$of which ${ }^{0} \widetilde{\mathcal{G}}^{-}$is a natural open substack.

The result of Helmke and Slodowy concerning elliptic singularities that we mentioned above is that there is a flat morphism $\mathcal{Z} \rightarrow[\widehat{\mathcal{Y}} / W]$ that is a minimally versal deformation of a simply elliptic singularity of degree $d$. We give a detailed proof of this as part of our main result, which is that there is also an analogue of the BGSS construction for the situation involving $\mathcal{E}$, as in the following theorem, which summarizes the results of the paper. The main result is given more concisely in Theorem 7.12. 
Theorem 1.2 Suppose that $G=E_{9-d}$.

(1) Let $\widetilde{\mathcal{Z}}^{\sharp}$ denote any one of $\widetilde{\mathcal{Z}}, \widetilde{\mathcal{Z}}^{ \pm}$. Then there is a commutative diagram

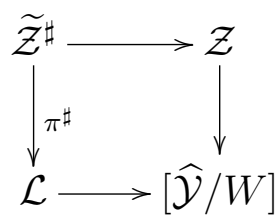

where $\pi^{\sharp}: \widetilde{\mathcal{Z}}^{\sharp} \rightarrow \mathcal{L}$ is semi-stable and smooth over $\mathcal{L}-0_{\mathcal{L}}$, the complement of the zero section $0_{\mathcal{L}}$.

(2) Suppose that 0 is a point of $0_{\mathcal{L}}$ lying over the origin $0_{\mathcal{Y}}$ of $\mathcal{Y}$. Then $\widetilde{\mathcal{Z}}_{0}=D_{0}+D_{1}+Q, \widetilde{\mathcal{Z}}_{0}^{ \pm}=D_{0}^{ \pm}+D_{1}^{ \pm}$, each $D_{0}^{\sharp} \cap D_{1}^{\sharp}$ is a copy of $\mathcal{E}, \mathcal{Z}_{0}$ is a cone over $\mathcal{E}$ of degree $d$ and $\widetilde{\mathcal{Z}}^{-}$is its minimal resolution. $Q$ is a copy of $\mathbb{P}^{1} \times \mathbb{P}^{1}$ and the morphism $\widetilde{\mathcal{Z}} \rightarrow \widetilde{\mathcal{Z}}^{+}$contracts $Q$ to a curve via a projection $Q \rightarrow \mathbb{P}^{1}$.

(3) When $\widetilde{\mathcal{Z}}^{\sharp}=\widetilde{\mathcal{Z}}^{-}$then the diagram is a type II simultaneous log resolution of $\mathcal{Z} \rightarrow[\widehat{\mathcal{Y}} / W]$.

(4) The rational map $\widetilde{\mathcal{Z}}^{+}-\rightarrow \widetilde{\mathcal{Z}}^{-}$is a sequence of flops and the centre of each flop is the total space of a smooth family of rational curves over $0_{\mathcal{L}}$.

(5) $\mathcal{Z} \rightarrow[\widehat{\mathcal{Y}} / W]$ is a miniversal deformation of a simply elliptic singularity of degree $d$ over $\operatorname{Spec} \mathbb{Q}$, and over $\operatorname{Spec} \mathbb{Z}[1 / d]$ if $d \leq 3$.

(6) The morphisms $\widetilde{\mathcal{Z}}^{\sharp} \rightarrow \mathcal{Z} \times_{[\widehat{\mathcal{Y}} / W]} \mathcal{L}$ are birational.

(7) The exceptional locus $\mathcal{D}_{1}^{-}$in $\mathcal{Z}^{-}$is the total space of a family $\mathcal{D}_{1}^{-} \rightarrow \mathcal{Y}=$ $0_{\mathcal{L}}$ of weak del Pezzo surfaces of degree $d$. In particular, the fibre over a point $(y, \xi)$ of the morphism $\mathcal{Z}^{-} \rightarrow \mathcal{Z} \times_{[\widehat{\mathcal{Y}} / W]} \mathcal{L}$, where $y$ is a point in the zero section $0_{\mathcal{L}}$ of $\mathcal{L}$, is a weak del Pezzo surface of degree $d$.

(8) Locally on $\mathcal{M}_{\text {Ell }}$ there is an isomorphism $\widetilde{\mathcal{Z}}^{-} \rightarrow \omega_{\mathcal{D}_{1}^{-} \mathcal{Y}}$.

The fibres of 1.27 are the Springer fibres of the title.

Figure 1 below shows the structure of the fibres $\widetilde{\mathcal{Z}}_{0}, \widetilde{\mathcal{Z}}_{0}^{ \pm}$and $\mathcal{Z}_{0}$.

The crucial part of the proof is Theorem 6.7, where we exhibit a surface $D_{1}$ in $\widetilde{\mathcal{G}}_{\mathcal{E}}$ whose transform under a natural flop is a weak del Pezzo surface. It follows (this is Corollary 6.18) that that the fibre of $\mathcal{Z} \rightarrow[\widehat{\mathcal{Y}} / W]$ over the origin of the affine space $[\widehat{\mathcal{Y}} / W]$ has a simply elliptic singularity. We then recover the result of Helmke and Slodowy, that $\mathcal{Z} \rightarrow[\widehat{\mathcal{Y}} / W]$ is a miniversal deformation of this singularity. In some sense, this is a "top down" approach which depends upon being able to recognize a singularity from its resolution.

Remark: In particular, we have made a non-trivial birational modification of $\widetilde{\mathcal{G}}_{\mathcal{E}}$, centred on a locus in the boundary $\widetilde{\mathcal{G}}_{\mathcal{E}}-\widetilde{\mathcal{G}}_{\mathcal{E}}^{s s}$, which is of a kind that is dictated by the needs of birational geometry but which has no obvious modular interpretation, in order to arrive at $\widetilde{\mathcal{Z}}^{-}$.

However, this is a common phenomenon.

For example, consider the coarse moduli space $A_{g}$ of principally polarized abelian varieties over $\mathbb{C}$ and its perfect compactification $A_{g}^{P}$. Then [SB16] the 


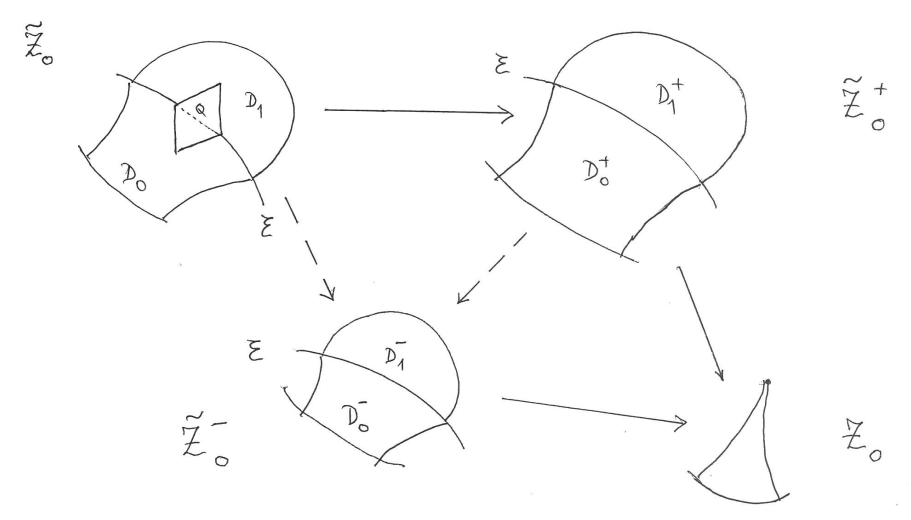

Figure 1: The fibres $\widetilde{\mathcal{Z}}^{\sharp}$ and $\mathcal{Z}_{0}$

exceptional locus of the $\mathbb{Q}$-divisor $12 M-D$, where $M$ is the bundle of modular forms of weight 1 and $D$ is the boundary, is the image of $A_{1}^{P} \times A_{g-1}^{P}$ in $A_{g}^{P}$, the locus of ppav's with an elliptic factor. So, if $7 \leq g \leq 10$, then $A_{g}^{P}$ has terminal singularities [AS16] and is of general type, while $K_{A_{g}^{P}} \sim(g+1) M-D$ is not nef. Therefore the first step in running the Minimal Model Program on $A_{g}^{P}$ is to contract and flip the extremal ray generated by the curve $A_{1}^{P} \times\{x\}$, where $x$ is a point $A_{g-1}^{P}$. This is a non-trivial birational modification which is dictated by the MMP and which is centred in a locus that penetrates the interior $A_{g}$ of $A_{g}^{P}$ that has no obvious modular interpretation, unless the notion of ppav can be modified.

It is important to point out that, beyond the work of Brieskorn and Looijenga mentioned above, and that of Pinkham $[\mathrm{P}]$, what we do builds very much on the results of Helmke and Slodowy, and, to an equal extent, on those of Friedman and Morgan [FM].

We will revisit the results of this paper in [GS], where amongst other things we will analyse the moduli stacks in a neighbourhood of infinity by using the Tate curve, give uniform descriptions of the combinatorics in terms of the affine Weyl groups and extend the main theorem of this paper to the cases where $5 \leq d \leq 9$.

Finally, if $H$ is a group then $\mathbb{B} H$ denotes its classifying stack and $[\mathcal{X}]$ denotes the geometric quotient of an algebraic stack $\mathcal{X}$ when that quotient exists. 


\section{Acknowledgements}

We are very grateful to Dougal Davis for many conversations on these matters and particularly for pointing out an error in our proof of the previous version of Proposition 6.4. We are also grateful to Igor Dolgachev for his question about unipotent singularities and to Simon Salamon for many kindnesses, which include drawing the picture.

\section{The BGSS construction}

To set the notation we begin by recalling a little of the machinery of reductive groups in a way that emphasizes the flag variety, and its intersection theory, of an affine group rather than the group itself. This approach is very well known, and details can be found in, for example, [MOSW].

As the starting point of the discussion, fix a smooth connected reductive ${ }^{2}$ algebraic group $G$ over a field $k$. Then [SGA3 XXII, Th. 5.8.1 and Cor. 5.8.3, pp. 228-230] there is an associated flag variety $F$ over $k$ that parametrizes the maximal soluble subalgebras (the Borel subalgebras) of $\mathfrak{g}=$ Lie $G$; it is constructed as a subscheme of the Grassmannian $G r(\mathfrak{g})$. It is a smooth projective $k$-variety and is homogeneous under the obvious $G$-action; it is maximal among the set of homogeneous projective $k$-varieties the stabilizers of whose points are smooth, and the stabilizer of each point of $F$ is a Borel subgroup (that is, a maximal connected reduced soluble subgroup of $G$ ). If $G$ has a Borel subgroup $B$ defined over $K$ then $F=G / B$, but we do not yet assume that such a subgroup exists; that is, we do not assume that $F$ has a $k$-point. By definition, this last condition is equivalent to $G$ being quasi-split.

Locally in the étale topology on Spec $k$ there is an array of projective homogeneous $G$-varieties, $2^{l}$ in number where $l$ is the semi-simple rank of $G$, and $G$-morphisms between them; in particular, there are $G$-maps $F \rightarrow F_{1}, \ldots, F \rightarrow F_{l}$ that are étale $\mathbb{P}^{1}$-bundles and there are $G$-maps $F \rightarrow X_{i}$ where $X_{i}$ is minimal, that is, of Picard number 1 . We shall assume that all these varieties, and the morphisms in the lattice, are defined over $k$; this assumption is fulfilled automatically if the Dynkin diagram attached to $G$ has no symmetries. Indeed, the Galois group that acts on the array of varieties above is a subgroup of the symmetry group of the Dynkin diagram. If $G$ is quasi-split, this is, by definition, the same as $G$ being split.

For each minimal variety $X_{i}$ there is a unique line bundle $\varpi_{i}$ on $X_{i} \otimes \bar{k}$ that is a positive generator of $\mathrm{Pic}_{X_{i} \otimes \bar{k}}$; we assume that $\varpi_{i}$ is defined over $K$. This condition holds automatically if $G$ is split. We also let $\varpi_{i}$ denote the pullback of $\varpi_{i}$ to $F$; then $\varpi_{1}, \ldots, \varpi_{l}$ are the fundamental dominant weights of $G$ and form

\footnotetext{
${ }^{2}$ This assumption was omitted in a previous version of this paper. Otherwise, as was pointed out by a referee, the field $k$ must be assumed to be perfect.
} 
a $\mathbb{Z}$-basis of $\mathrm{Pic}_{F}$. (So, for groups of type $E_{8}$ the bundles $\varpi_{i}$ are always defined over $k$, because here the root lattice equals the weight lattice.) They also span the nef cone of $F$, which is also the effective cone of $F$. The semi-simple rank of $G$ is the Picard number of $F$.

Set $\alpha_{j}=T_{F / F_{j}}$, the relative tangent bundle. This is a $G$-linearized line bundle, so defines a class in $\operatorname{Pic}_{F}^{G}$, the group of classes of $G$-linearized line bundles on $F$. (The rank of this group is the rank of $G$.) These classes are the simple roots of $G$. The simple coroots are $\alpha_{1}^{\vee}, \ldots, \alpha_{l}^{\vee}$ where $\alpha_{i}^{\vee}$ is the class of a fibre of $F \rightarrow F_{i}$; such a curve is a conic (a smooth curve of genus zero).

So some of the various intersection numbers between these curves and divisor classes are given by $\left(\alpha_{i}^{\vee}, \varpi_{j}\right)=\delta_{i j}$ and $\left(\alpha_{i}^{\vee}, \alpha_{i}\right)=2$.

The numbers $1, \ldots, l$ are the nodes of the associated Coxeter-Dynkin diagram $D$; the edges of $D$ are labelled in a way that is determined by the numbers $\left(\alpha_{i}^{\vee}, \alpha_{j}\right)$. This diagram leads to a Coxeter system $\left(W, S=\left\{s_{1}, \ldots, s_{l}\right\}\right)$ acting on $\operatorname{Pic}^{G} F$ in the usual way: $s_{i}(x)=x+\left(x \cdot \alpha_{i}^{\vee}\right) \alpha_{i}$. There is also a root datum $\left(M, M^{\vee}, \Delta, \Delta^{\vee}\right)$ derived from this picture: $M=\operatorname{Pic}_{F}^{G}, \Delta$ is the $W$-orbit of the set of simple roots and $\Delta^{\vee}$ is the $W$-orbit of the set of simple coroots. More intrinsically, the roots arise as the $G$-linearized line bundles in a natural filtration of the tangent bundle $T_{F}$ and the coroots appear as the classes of curves in the Bruhat decomposition of $F$. Conversely, each root datum leads to a unique split reductive group defined over $\mathbb{Z}$. (A smooth affine group $G$ over a base $S$ is reductive if all its geometric fibres are reductive.)

Now assume that $G$ is quasi-split, so split. Fix a $k$-point of $F$ and rigidify all line bundles and $G$-linearized line bundles on $F$ at this point. Define the weight lattice $P$ of $G$ by $P=\operatorname{Pic}_{F}^{G}$. Then there are universal torsors $\mathcal{T} \rightarrow F$ and $\mathcal{T}^{G} \rightarrow F$ under tori $T$ and $T^{G}$, respectively, whose character groups are $\mathbb{X}^{*}(T)=\operatorname{Pic}_{F}$ and $\mathbb{X}^{*}\left(T^{G}\right)=P$. Since $G$ is reductive it acts effectively on $\mathcal{T}^{G}$.

If the forgetful homomorphism $\operatorname{Pic}_{F}^{G} \rightarrow \mathrm{Pic}_{F}$ is injective then $G$ is semi-simple and if it is an isomorphism then $G$ is simply connected. In this case the maps $F \rightarrow F_{i}$ are Zariski $\mathbb{P}^{1}$-bundles. A semi-simple group is simple if its Dynkin diagram is connected.

Remark: It is clear from this that in fact a reductive group does not give rise only to a root datum, but rather to a pinned root datum [SGA3 XXIII, 1.5]. On the other hand, a del Pezzo surface $S$ gives rise to a root datum $\left(M, M^{*}, R, R^{*}\right)$ in three ways: $M$ is one of $K_{S}^{\perp}, \mathrm{NS}(S) / \mathbb{Z} \cdot\left[K_{S}\right]$ and $\mathrm{NS}(S)$ and $R=R^{*}$ is the set of $(-2)$-vectors in $\mathrm{NS}(S)$ or the image of that set modulo $\mathbb{Z}$. $\left[K_{S}\right]$, but this datum is not naturally pinned, because of the existence of monodromy on $\operatorname{NS}(S)$. This explains why the construction to be given in this paper leads to del Pezzo surfaces that are split, in the sense that this monodromy is trivial. To put it another way, when the Dynkin diagram has no symmetry, as in the case of $E_{8}$, there is no monodromy on $\operatorname{NS}(F)=\operatorname{Pic}_{F}$ and whether $F$ has a $k$-point is a non-trivial issue. However, del Pezzo surfaces of degree 1 always have a naturally defined $k$-point 
(the base point of the anticanonical system) while the monodromy is significant.

For the rest of this paper $G$ will be a split simply connected simple reductive group.

Define the torus $T$ by the formula $\mathbb{X}^{*}(T)=\operatorname{Pic}_{F}^{G}$, or $T=\operatorname{Hom}\left(\operatorname{Pic}_{F}^{G}, \mathbb{G}_{m}\right)$. By assumption, $T$ is split but is not necessarily isomorphic to a subgroup of $G$.

Next, we recall the BGSS construction.

Define the incidence subvariety $\widetilde{G}$ of $G \times F$ by

$$
\widetilde{G}=\{(g, x) \mid g(x)=x\} .
$$

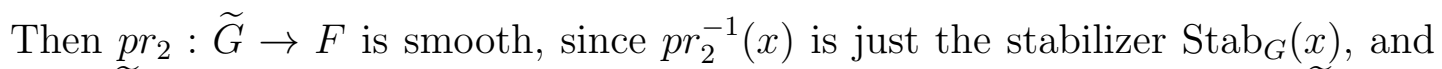
$p r_{1}: \widetilde{G} \rightarrow G$ is projective. Moreover, there is a smooth morphism $\pi: \widetilde{G} \rightarrow T$ given by

$$
\pi(g, x)(L)=\left(\phi: g^{*} L \rightarrow L\right)(x),
$$

where the isomorphism $\phi: g^{*} L \rightarrow L$ is part of the data of the $G$-linearization of the line bundle $L$.

Remark: Fix a $k$-point $x$ on $F$. Take $B=p r_{2}^{-1}(x)=\operatorname{Stab}_{G}(x)$. Then $\pi$ restricts to a surjective homomorphism $B \rightarrow T$. This homomorphism is split, and choosing a splitting of it gives the standard set-up $T \hookrightarrow B \hookrightarrow G$ of the theory of reductive groups. The torus $T$ that we have taken is an abstract Cartan subgroup.

In fact, $\left(p r_{2}, \pi\right): \widetilde{G} \rightarrow F \times T$ is smooth; each fibre is a translate of the unipotent radical of a Borel subgroup of $G$.

Let $\widetilde{G} \rightarrow X \rightarrow G$ be the Stein factorization of $p r_{1}$. Then $X=\operatorname{Spec} \Gamma\left(\widetilde{G}, \mathcal{O}_{\widetilde{G}}\right)$ is the affine hull of $\widetilde{G}$, so that $\pi: \widetilde{G} \rightarrow T$ factors through $X$.

From the definitions of $W$ and $T$ there is an action of $W$ on $T$ and there is a commutative square

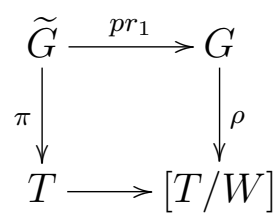

The key point to be proved here is that the composite morphism $\widetilde{G} \rightarrow T \rightarrow[T / W]$ factors through $p r_{1}: \widetilde{G} \rightarrow G$; this can be verified over $\bar{k}$, where it follows from (1) the existence of a maximal torus $T_{1}$ in $G$ that is isomorphic to $T$ and (2) the fact that the pair $\left(T_{1}, N_{G}\left(T_{1}\right)\right)$ is a slice to the adjoint action of $G$ on itself.

Proposition 2.1 The morphism $\pi: \widetilde{G} \rightarrow T$ is relatively symplectic and the canonical class $K_{\widetilde{G}}$ is trivial.

PROOF: The smooth morphism $p r_{2}: \widetilde{G} \rightarrow F$ gives, at each point $y$ of $\widetilde{G}$, an exact sequence

$$
0 \rightarrow \mathfrak{b} \rightarrow T_{\widetilde{G}}(y) \rightarrow \mathfrak{g} / \mathfrak{b} \rightarrow 0
$$


So there is an exact sequence

$$
0 \rightarrow \mathfrak{u} \rightarrow T_{\pi}(y) \rightarrow \mathfrak{g} / \mathfrak{b} \rightarrow 0,
$$

where $\mathfrak{u}$ is the Lie algebra of the unipotent radical $U$ of $B$. The Killing form shows that this sequence is self-dual, which gives the relative symplectic structure in question. Since $K_{T} \sim 0$, Then taking the Pfaffian of the relative symplectic structure gives the triviality of the relative canonical class $K_{\widetilde{G} / T}$; since $K_{T}$ is trivial, the proposition is proved.

From the viewpoint of algebraic surfaces, their singularities and their moduli, the significance of this diagram is that, if the edges of the Coxeter-Dynkin diagram are unlabelled (that is, if the group is of type $A D E$ ), then, the localization of the unipotent variety $U n i(G)$ at the geometric generic point of the subregular unipotent orbit in $G$ has a du Val singularity of the same type as $G$ and the morphism $\rho: G \rightarrow[T / W]$ has normal fibres (this can be proved over $\bar{k}$ as a corollary of Steinberg's theorem that in that context $\rho$ has a section). Moreover, except in bad characteristic (taken to include any prime divisor of $n+1$ in the case of type $\left.A_{n}\right), \rho$ yields a miniversal deformation of this singularity and $\pi: \widetilde{G} \rightarrow T$ gives a miniversal deformation of the minimal resolution of the singularity.

\section{BGSS for semi-stable bundles over uniformiz- able elliptic curves}

Fix an elliptic curve $E$ over a field $k$ and a simply connected reductive group $G$ over $k$. The word "bundle" will imply "locally trivial in the étale topology". A principal $G$-bundle $\Xi \rightarrow E$ gives rise to an $F$-bundle $\mathcal{F}_{\Xi}=\mathcal{F}=\Xi \times^{G} F$ and line bundles $L_{\varpi}=\Xi \times^{G} \varpi$ on $\mathcal{F}$ for any weight $\varpi$. Conversely, suppose that $p: \mathcal{F} \rightarrow E$ is an $F$-bundle and that $L_{\varpi_{1}}, \ldots, L_{\varpi_{l}}$ are line bundles on $\mathcal{F}$ such that $L_{\varpi_{i}}$ restricts to $\varpi_{i}$ on each geometric fibre of $p$. Then there is a reductive and simply connected group scheme $G_{1}$ over $E$ defined as the automorphism group scheme of $\mathcal{F}$ and all the given line bundles. If the sheaves $p_{*} L_{\varpi_{i}}$ are free on $E$ then $G_{1}$ is constant and pulls back from a $k$-form of $G$.

Fix $\Xi \rightarrow E$. Every section $\sigma$ of $\mathcal{F} \rightarrow E$ defines a co-character $[\sigma]$ by $([\sigma], \varpi)=$ $\sigma \cdot c_{1}\left(L_{\varpi}\right)$. We say that $[\sigma] \leq 0$ if $[\sigma] . \varpi_{i} \leq 0$ for every fundamental dominant weight $\varpi_{i}$ and $[\sigma]<0$ if $[\sigma] \leq 0$ and $\sigma \neq 0$.

Definition 3.1 A G-bundle $\Xi \rightarrow E$ is semi-stable if ad $\Xi$ is semi-stable as a coherent sheaf on $E$ and is unstable if it is not semi-stable.

This is well known ([HS2] Theorem 1.1.2) to be equivalent to other definitions. In particular, $\Xi$ is semi-stable if and only if, for every section $\sigma$ of $\mathcal{F}_{\Xi} \rightarrow E$, the associated cocharacter $[\sigma]$ satisfies $[\sigma] \geq 0$.

Recall also Theorem 1.3.1 of [HS2]: for any unstable principal $G$-bundle $\Xi \rightarrow$ $E$, there is a proper parabolic subgroup $P$ of $G$, a Levi subgroup $\Lambda$ of $P$ and a 
principal $\Lambda$-bundle $\lambda \rightarrow E$ such that $\lambda$ is semi-stable, $\Xi=\lambda \times^{\Lambda} G \rightarrow E$ and $\Lambda$ is maximal among Levi subgroups with this property. Moreover, $\Lambda$ is unique up to $G$-conjugacy and $\lambda$ is determined up to isomorphism of principal bundles.

This is proved as a consequence of a unique decomposition ad $\Xi=\mathfrak{l} \oplus \underline{\mathfrak{u}} \oplus \underline{\mathfrak{u}}^{*}$ of vector bundles, where $\underline{\mathfrak{l}}=\operatorname{ad} \lambda$ consists of summands of degree $0, \underline{\mathfrak{u}}$ consists of summands of positive degree and $\underline{\mathfrak{l}} \oplus \underline{\mathfrak{u}}=\operatorname{ad}\left(\lambda \times{ }^{\Lambda} P\right)$. In turn, this follows from Atiyah's classification of vector bundles on an elliptic curve.

Let $\mathcal{G}_{E}^{s s}$ denote the stack of semi-stable $G$-bundles on $E$ and $\widetilde{\mathcal{G}}_{E}^{s s}$ the stack whose objects are pairs $(\Xi, \sigma)$ where $\Xi \in \mathrm{Ob}_{E}^{s s}$ and $\sigma$ is a section of the $F$ bundle $\mathcal{F}_{\Xi}=\Xi \times^{G} F \rightarrow E$ whose associated cocharacter $[\sigma]$ is zero.

Lemma 3.2 $\widetilde{\mathcal{G}}_{E}^{s s}$ is naturally isomorphic to the stack $\mathcal{B}_{E}^{0}$ of $B$-bundles $\beta \rightarrow E$ whose associated $T$-bundle $\beta \times{ }^{B} T \rightarrow E$ is of degree 0 .

PROOF: This follows from the tautology that giving a section of $\mathcal{F}_{\Xi}$ is the same as giving a reduction of $\Xi$ to a $B$-bundle.

In the rest of this section we assume, until the end of the proof of Theorem 3.11 , that the elliptic curve $E$ is uniformizable; that is, that we can write $E=$ $\mathbb{G}_{m} /\left\langle q^{\mathbb{Z}}\right\rangle$. For example, this holds if either $k=\mathbb{C}$ or $k$ is an algebraically closed complete valued field, char $k \neq 2$ and $j(E)$ is not an integer of $k$ [BGR, 9.7]. If char $k=2$ then there do exist uniformizable curves, derived from the Tate curve. We will return to the Tate curve in the future.

Fix a co-ordinate $z$ on $\mathbb{G}_{m}$; then we claim that there is a morphism $\tilde{f}: \widetilde{G} \rightarrow \widetilde{\mathcal{G}}_{E}^{s s}$ defined by

$$
\tilde{f}(g, x)=\left(\left(G \times \mathbb{G}_{m}\right) /\langle\gamma\rangle,\{x\} \times E\right)=(\Xi, \sigma),
$$

where $\gamma(h, z)=\left(g h g^{-1}, q z\right)$. The $F$-bundle $\mathcal{F}_{\Xi}$ associated to the $G$-bundle $\Xi=$ $\tilde{f}(g, x)$ is the quotient $\left(F \times \mathbb{G}_{m}\right) /\langle\gamma\rangle$ where $\gamma(y, z)=(g(y), q z)$ for any point $y \in F$, so that indeed the image of $\{x\} \times E$ is a section of $\mathcal{F}_{\Xi}$.

To establish the claim that $\tilde{f}(g, x)$ is a point of $\widetilde{\mathcal{G}}_{E}^{s s}$, note that the cocharacter $[\sigma]$ of the section $\sigma=\{x\} \times E$ of $\mathcal{F}_{\Xi}$ vanishes by continuity, because after specializing to the identity element $g=1$ of $G$ it is certainly zero.

Lemma 3.3 There is a commutative square

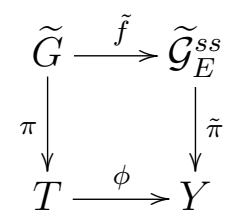

where $\phi$ is the unramified covering induced from the uniformization $\mathbb{G}_{m} \rightarrow E \cong$ $\operatorname{Pic}^{0}(E)$.

PROOF: This follows immediately from the definitions of the morphisms involved. 
Lemma $3.4 \tilde{\pi}$ is smooth.

PROOF: By Lemma 3.2 we can identify $\widetilde{\mathcal{G}}_{E}^{\text {ss }}$ with the stack $\mathcal{B}_{E}^{0}$, and then $\tilde{\pi}$ is the morphism that maps a $B$-bundle $\beta \rightarrow E$ to its associated $T$-bundle $\tau=$ $\beta \times^{B} T \rightarrow E$. The smoothness of $\tilde{\pi}$ follows from the surjectivity of the map $H^{1}(E, \operatorname{ad} \beta) \rightarrow H^{1}(E, \operatorname{ad} \tau)$ of tangent spaces, which follows in turn from the surjectivity of the morphism ad $\beta \rightarrow$ ad $\tau$ of coherent sheaves on $E$ and the fact that $E$ is 1-dimensional.

Lemma $3.5[T / W] \rightarrow[Y / W]$ is étale in an analytic neighbourhood of $0_{Y}$ and $[Y / W]$ is smooth in a Zariski neighbourhood of $0_{Y}$.

PROOF: The morphism $\phi: T \rightarrow Y$ is the quotient by the group $P$, which acts freely on $T$, so $\phi$ is étale. Moreover, the group $\widetilde{W}=P \rtimes W$ acts on $T$ and the stabilizer $\operatorname{Stab}_{\widetilde{W}}\left(1_{T}\right)$, where $1_{T}$ is the identity point of $T$, is $W$, which equals $\operatorname{Stab}_{W}\left(0_{Y}\right)$. The first part of the lemma is now established, and the rest follows from the smoothness of $[T / W]$.

Now consider the commutative diagram ("the basic comparison")

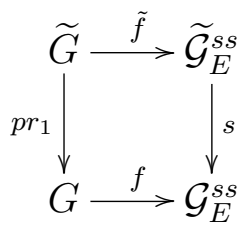

where $f(g)=\left(G \times \mathbb{G}_{m}\right) /\langle\gamma\rangle$ with $\gamma(h, z)=\left(g h g^{-1}, q z\right)$. We shall show, in Theorem 3.8 below, that this is Cartesian when restricted to the analytic neighbourhood $\mathcal{U}$ of the identity element $e$ of $G$ that is described in the next Lemma.

Lemma 3.6 There is an analytic (classical or rigid) open neighbourhood $\mathcal{U}$ of $e$ in $G$ on which $f$ is étale, and so surjective.

PROOF: The codifferential $f^{*}$ is, via Serre duality, a map $H^{0}\left(E,(\operatorname{ad} \Xi)^{*}\right) \rightarrow \mathfrak{g}^{*}$. For the trivial $G$-bundle $\Xi=E \times G$ this is, by inspection, an isomorphism. So $f$ is smooth at $e$, and the result follows.

Note that $\mathcal{U}$ maps to an analytic (classical or rigid) neighbourhood of the trivial bundle $E \times G$ in $\mathcal{G}_{E}^{s s}$.

Recall that $p r_{1}$ and $s$ are projective, generically finite and dominant. In fact, $s$ is finite over the locus $\widetilde{\mathcal{G}}_{E, \text { reg }}^{\text {ss }}$ of regular semi-stable bundles (those whose automorphism group is of minimal dimension, namely the rank of $G$ ).

Lemma $3.7 \operatorname{deg} s=\# W=\operatorname{deg} p r_{1}$.

PROOF: We can assume that there are subgroups $T \hookrightarrow B \hookrightarrow G$ as in the usual set-up for split reductive groups, with a surjection $B \rightarrow T$. Then there is a 
commutative diagram

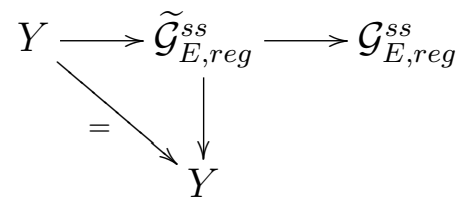

arising from the identification made above of $\widetilde{\mathcal{G}}_{E}^{s s}$ with the stack $\mathcal{B}_{E}^{0}$. Now $\mathcal{G}_{E, \text { reg }}^{s s}$ has a geometric quotient that can be identified with $[Y / W]$ and the fibres of $\widetilde{\mathcal{G}}_{E, \text { reg }}^{\text {ss }} \rightarrow Y$ are point over the regular locus in $Y$. That is, over the maximal open subvariety $Y^{0}$ of $Y$ on which $Y$ acts freely.

In other words, the square

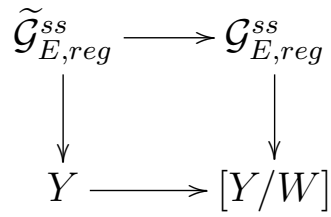

is Cartesian when restricted to $Y^{0}$ and this is enough.

Theorem 3.8 The square

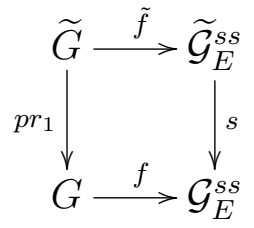

in the "basic comparison" is Cartesian when restricted to the neighbourhood $\mathcal{U}$ of $e$ in $G$.

PROOF: Consider the diagram

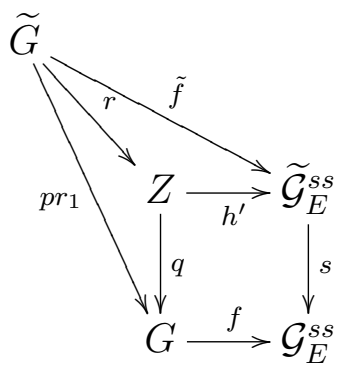

where $Z$ is the fibre product. Since $\operatorname{deg} p r_{1}=\operatorname{deg} s$, it follows that $r$ is birational; clearly, $r$ is proper. Also, $h^{\prime}$ is smooth on $\mathcal{U}_{1}=q^{-1}(\mathcal{U})$, since $f$ is smooth on $\mathcal{U}$, so that $\mathcal{U}_{1}$ is smooth. Set $\mathcal{U}_{2}=r^{-1}\left(\mathcal{U}_{1}\right)$, an open subvariety of $\widetilde{G}$. So $r: \mathcal{U}_{2} \rightarrow \mathcal{U}_{1}$ is a proper birational morphism of smooth analytic $k$-varieties.

By Proposition 2.1 the canonical divisor class $K_{\widetilde{G}}$ is trivial, and then $K_{\mathcal{U}_{2}} \sim 0$. 
Lemma 3.9 If $g: X \rightarrow Y$ is a proper birational morphism of smooth (rigid analytic or classical) $k$-varieties and $K_{X} \sim 0$, then $g$ is an isomorphism.

PROOF: This is very well known in characteristic zero but possibly less so in positive characteristic. So we give a proof.

There is an open subvariety $V$ of $Y$ whose complement is of codimension at least 2 over which $g$ is an isomorphism. Therefore the trace map $t r: g_{*} \omega_{X} \rightarrow \omega_{Y}$ is an isomorphism on $V$; since $g_{*} \omega_{X} \cong \mathcal{O}_{Y}$ it follows that $t r$ is an isomorphism and that $\omega_{Y} \cong \mathcal{O}_{Y}$. Then the natural homomorphism $g^{*} \omega_{Y} \rightarrow \omega_{X}$ is also an isomorphism. Therefore the determinant of the derivative $d g: T_{X} \rightarrow g^{*} T_{Y}$ is an isomorphism, and so $d g$ is an isomorphism. Therefore $g$ is smooth. Since it is also proper and birational it is an isomorphism.

In particular, the restriction of $r$ to $\mathcal{U}_{2}$ is an isomorphism and we are done.

Corollary 3.10 $s: \widetilde{\mathcal{G}}_{E}^{s s} \rightarrow \mathcal{G}_{E}^{s s}$ is proper and surjective.

PROOF: By Theorem 3.8 the morphism $s$ is surjective in a neighbourhood of the trivial bundle in $\mathcal{G}^{s s}$. The properness follows from the valuative criterion and the fact that, if $\left\{\Xi_{t}\right\}$ is a family of semi-stable $G$-bundles over $E$, then a section $\sigma_{t}$ of $\mathcal{F}_{\Xi_{t}}$ whose cocharacter $\left[\sigma_{t}\right]$ vanishes can only specialize to a section $\left[\sigma_{0}\right]$ of $\mathcal{F}_{\Xi_{0}}$ such that $\left[\sigma_{0}\right] \leq 0$. Since $\Xi_{0}$ is semi-stable, $\left[\sigma_{0}\right]=0$.

Theorem 3.11 In a neighbourhood of the origin in $[Y / W]$ the squares
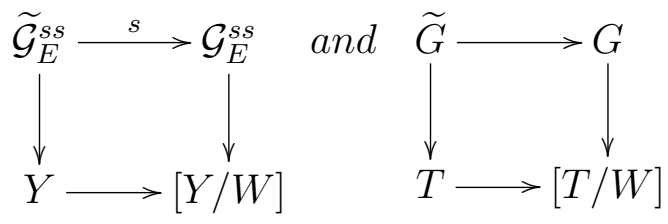

are smoothly equivalent.

PROOF: Define $\mathcal{H}=\mathcal{G}_{E}^{s s} \times_{[Y / W]}[T / W]$ and $\widetilde{\mathcal{H}}=\widetilde{\mathcal{G}}_{E}^{s s} \times_{Y} T$, so that the square

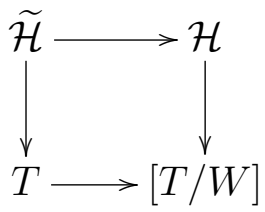

is the pull-back under $[T / W] \rightarrow[Y / W]$ of the square

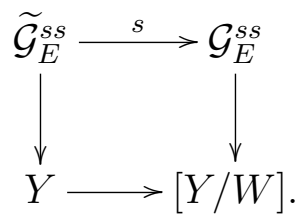


We have shown that, near $e$, the square

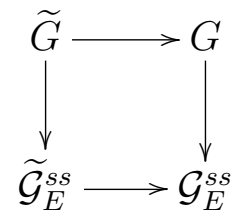

is Cartesian; the same argument shows that, near $e, G \rightarrow \mathcal{H}$ is smooth and that

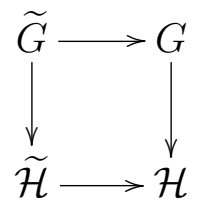

is Cartesian in a neighbourhood of $e$. So, in a neighbourhood of $e$,

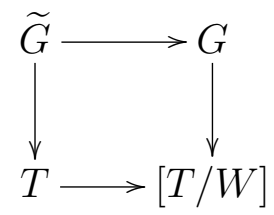

is smoothly equivalent to

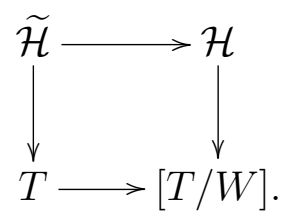

Comparing these two descriptions of the last square gives the result.

We shall see later that this result fails when $E$ is supersingular in characteristics 2,3 and 5 and $G=E_{8}$.

We can now tie up some loose ends concerning semi-stability. We no longer assume the curve $E$ to be uniformizable.

We have recovered, as Corollary 3.10, the well known fact that the first projection $s: \widetilde{\mathcal{G}}_{E}^{s s} \rightarrow \mathcal{G}_{E}^{s s}$ is surjective and proper when the base is $\operatorname{Spec} \mathbb{C}$. This is true over any base.

Lemma 3.12 The projection $s: \widetilde{\mathcal{G}}_{\mathcal{E}}^{s s} \rightarrow \mathcal{G}_{\mathcal{E}}^{s s}$ is surjective and projective.

PROOF: It follows from Corollary 3.10 that $s: \widetilde{\mathcal{G}}_{E}^{s s} \rightarrow \mathcal{G}_{E}^{s s}$ is surjective if $E$ is defined over $\mathbb{C}$, and so over any field of characteristic zero. Suppose then that $\Xi$ is a semi-stable $G$ bundle over $E$ in characteristic $p$; lift $E$ and $\Xi$ to $\widetilde{\Xi} \rightarrow \widetilde{E}$ in characteristic zero. There is then a section $\tilde{\sigma}$ of $\mathcal{F}_{\widetilde{\Xi}}$ with $[\tilde{\sigma}]=0$. Specializing back to characteristic $p$ gives a section $\sigma$ of $\mathcal{F}_{\Xi}$ in characteristic $p$ with $[\sigma] \leq 0$; since $\Xi$ is semi-stable, $[\sigma]=0$.

So $s$ is surjective. Properness follows from the valuative criterion in the same way and then projectivity is a consequence of the projectivity of the Hilbert scheme. 
Proposition 3.13 If the base is an algebraically closed field, then $\Xi$ is semistable if and only if $\mathcal{F}_{\Xi}$ has a section $\sigma$ with $[\sigma]=0$ and $\Xi$ is unstable if and only if $\mathcal{F}_{\Xi}$ has a section $\sigma$ with $[\sigma]<0$.

PROOF: Suppose that $\Xi$ is semi-stable. Then the existence of a suitable $\sigma$ is the surjectivity to which we have just alluded. Conversely, the existence of a section $\sigma$ such that $[\sigma]=0$ leads to a description of ad $\Xi$ as an extension of line bundles of degree 0, which implies its semi-stability.

According to [HS3], 3.3, every unstable $G$-bundle $\Xi_{0}$ can be deformed to a semi-stable bundle $\Xi_{t}$. Then $\mathcal{F}_{\Xi_{t}}$ has a section $\sigma_{t}$ with $\left[\sigma_{t}\right]=0$; specializing to $t=0$ gives a section $\sigma_{0}$ of $\mathcal{F}_{\Xi_{0}}$ with $\left[\sigma_{0}\right] \leq 0$. Since $\Xi_{0}$ is unstable, $\left[\sigma_{0}\right] \neq 0$.

Remark: There is a special case of Theorem 1.3.1 of [HS2] that we can now spell out.

Suppose that $\Xi$ can be reduced to a semi-table $\Lambda$-bundle where $\Lambda$ is a Levi subgroup of a maximal parabolic subgroup $P$ of $G$. Let $\Xi_{P}=\Xi / P=\Xi \times^{G}$ $G / P \rightarrow E$ be the associated $(G / P)$-bundle. Then $\Xi_{P} \rightarrow E$ has a section $\sigma$ whose associated cocharacter $[\sigma]$ is a negative integer multiple of $\alpha^{\vee}$, where $\alpha^{\vee}$ is the simple coroot belonging to $P$.

\section{Some relative compactifications of $\widetilde{\mathcal{G}}_{\mathcal{E}}^{s s}$}

We denote by $\mathcal{T}^{0}$ the stack of $T$-bundles over $\mathcal{E}$ of multi-degree zero, so that $\mathcal{T}^{0} \cong \mathcal{Y} \times \mathbb{B} T$, where $\mathcal{Y}=\operatorname{Hom}(P, \mathcal{E})$ and, for a group $H, \mathbb{B} H=\{*\} / H$ is the classifying stack of $H$-bundles. There are morphisms $s: \widetilde{\mathcal{G}}_{\mathcal{E}}^{s s} \rightarrow \mathcal{G}_{\mathcal{E}}^{s s}$ and $\tilde{\pi}: \widetilde{\mathcal{G}}_{\mathcal{E}}^{s s} \rightarrow \mathcal{Y}$ given by $\tilde{\pi}(\Xi, \sigma)(\varpi)=\left.L_{\varpi}\right|_{\sigma}$ and these stacks and morphisms fit into a commutative square, analogous to the square in the BGSS construction,

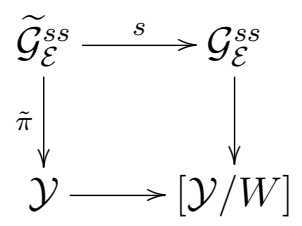

where $s=p r_{1}$ is projective (in particular, representable), by Proposition 4.1 below, and $[\mathcal{Y} / W]$ is the geometric quotient, relative to $\mathcal{M}_{E l l}$, of the open substack $\mathcal{G}_{\mathcal{E}, \text { reg }}^{\text {ss }}$ of regular semi-stable bundles. Here "regular" means that the automorphism group has minimal dimension $l$, the rank of $G$.

It is well known that the classifying morphism $\mathcal{G}_{\mathcal{E}, \text { reg }}^{\text {ss }} \rightarrow[\mathcal{Y} / W]$ extends to a morphism $\gamma: \mathcal{G}_{\mathcal{E}}^{s s} \rightarrow[\mathcal{Y} / W]$ given by sending a bundle to its $S$-equivalence class.

We now recall various relative compactifications of $\widetilde{\mathcal{G}}_{\mathcal{E}}^{s s}$. There are several that are relevant, but the most useful for us here, because of its smoothness properties, will be denoted by $\widetilde{\mathcal{G}}_{\mathcal{E}}$ or $\widetilde{\mathcal{G}}_{\mathcal{E}}^{K M}$; it is based on the stack of stable maps introduced by Kontsevich (although it is also appropriate to attach the name of Mori). That 
is, it relies on enlarging the source of a map, rather than on Drinfel'd's idea, which we now recall, of enlarging the target.

Define $R=\oplus_{\varpi} H^{0}(F, \varpi)$, the Cox ring of $F$, the sum being taken over all dominant weights. Let $T$ denote the torus introduced previously and consider the singular and non-separated stack $\bar{F}=(\operatorname{Spec} R) / T$. There is an open $G$ equivariant embedding $F \hookrightarrow \bar{F}$ and Drinfel'd's idea, which is described in Section 1 of $[\mathrm{BG}]$, and recalled in more detail below, is to embed $\mathcal{F}_{\Xi}$ into the $\bar{F}$-bundle $\Xi \times^{G} \bar{F} \rightarrow E$ and consider sections of this. Of course, any projective homogeneous $G$-variety $X$ has a similar enlargement $X \hookrightarrow \bar{X}$. This Kontsevich-Mori compactification turns out to be slightly wrong for our purposes (roughly speaking, it needs to be contracted and then flopped) but the extra information that it contains turns out to be crucial for the proof of our main result. The Drinfel'd compactification $\widetilde{\mathcal{G}}_{\mathcal{E}}^{D}$, on the other hand, is too small and too singular.

Let $\mathcal{C}_{\text {pre }} \rightarrow$ Spec $\mathbb{Z}$ denote the stack whose objects over a point $S$ are 1-marked pre-stable curves $C \rightarrow S$ whose canonical model is an elliptic curve over $S$. So, if $S$ is a geometric point, then $C$ is reduced with normal crossings, its dual graph is a tree, every irreducible component except one, say $C_{1}$, a a copy of $\mathbb{P}^{1}$ and $C_{1}$ is an elliptic curve. We refer to these as "elliptic curves with rational tails" and to $\mathcal{C}_{\text {pre }}$ as the stack of elliptic curves with tails. Note that $\mathcal{C}_{\text {pre }}$ is a smooth stack over $\mathbb{Z}$ and that its discriminant, the locus of singular curves, is a divisor $\Delta=\Delta\left(\mathcal{C}_{\text {pre }}\right)$ in $\mathcal{C}_{\text {pre }}$ with normal crossings. The complement $\mathcal{C}_{\text {pre }}-\Delta$ is naturally isomorphic to $\mathcal{M}_{\text {Ell }}$ and there is a retraction $\mathcal{C}_{\text {pre }} \rightarrow \mathcal{M}_{\text {Ell }}$ given by sending a pre-stable curve to its canonical model. This retraction is smooth, since it is surjective on tangent spaces.

Let $\widetilde{\mathcal{G}}_{\mathcal{E}}=\widetilde{\mathcal{G}}_{\mathcal{E}}^{K M}$ be the stack whose objects over a scheme $S$ are triples $(\Xi, C, \sigma$ : $\left.C \rightarrow \Xi \times{ }^{G} F\right)$, where $\Xi$ is a $G$-bundle over $E=\mathcal{E} \times \times_{\mathcal{M}_{E l l}} S, C$ is an $S$-object of $\mathcal{C}_{\text {pre }}$ and $\sigma$ is a stable map (in the sense of Kontsevich) such that the composite $C \rightarrow \Xi \times{ }^{G} F \rightarrow E$ is the contraction of $C$ to its canonical model and $\operatorname{deg} \sigma^{*} L_{\varpi_{i}}=0$ for each fundamental dominant weight $\varpi_{i}$. (Here, degree means total degree, the sum of the degrees on each component of $C$.) Let $s: \widetilde{\mathcal{G}} \rightarrow \mathcal{G}$ be the forgetful map $\left(\Xi, C, \sigma: C \rightarrow \Xi \times^{G} F\right) \mapsto \Xi$.

Proposition 4.1 (1) $s: \widetilde{\mathcal{G}}_{\mathcal{E}} \rightarrow \mathcal{G}_{\mathcal{E}}$ is proper and has finite relative automorphism group schemes.

(2) $p r_{2}: \widetilde{\mathcal{G}_{\mathcal{E}}} \rightarrow \mathcal{C}_{\text {pre }}$ is smooth.

(3) There is a smooth morphism $\pi: \widetilde{\mathcal{G}}_{\mathcal{E}} \rightarrow \mathcal{Y}$ that extends the morphism $\tilde{\pi}: \widetilde{\mathcal{G}}_{\mathcal{E}}^{s s} \rightarrow \mathcal{Y}$ described previously.

(4) $\left(p r_{2}, \pi\right): \widetilde{\mathcal{G}}_{\mathcal{E}} \rightarrow \mathcal{C}_{\text {pre }} \times{ }_{\mathcal{M}_{E l l}} \mathcal{Y}$ is also smooth.

(5) $s$ is representable when restricted to the locus in $\widetilde{\mathcal{G}}_{\mathcal{E}}$ where, on each geometric component of $C$, either $\sigma$ is of degree 1 onto its image or $\sigma$ is constant.

PROOF: The only things which are neither obvious nor well known are (3) and (4). 
For (3), it is enough to consider the universal curve $\Gamma \rightarrow \mathcal{C}_{\text {pre }}$ and then notice that the contraction $\Gamma \rightarrow \mathcal{E} \times{ }_{\mathcal{M}_{E l l}} \mathcal{C}_{\text {pre }}$ is a projective and birational morphism of smooth stacks. It is well known that for such a morphism there is a blowingdown morphism from $\mathbb{G}_{m}$-bundles on $\Gamma$ to $\mathbb{G}_{m}$-bundles on $\mathcal{E} \times{ }_{\mathcal{M}_{E l l}} \mathcal{C}_{\text {pre }}$, given by pushing forward divisor classes; this extends to a blowing-down morphism from $T$-bundles on $\Gamma$ to $T$-bundles on $\mathcal{E} \times{ }_{\mathcal{M}_{E l l}} \mathcal{C}_{\text {pre }}$ for any split torus $T$, by induction on the rank of $T$. So $\pi$ exists; its smoothness is an immediate consequence of the fact that curves are 1-dimensional.

(4): Suppose that $k$ is an algebraically closed field and that $C$ is a $k$-point of $\mathcal{C}_{\text {pre }}$ whose canonical model is the elliptic curve $E$. Since $H^{1}\left(E, \mathcal{O}_{E}\right) \rightarrow$ $H^{1}\left(C, \mathcal{O}_{C}\right)$ is an isomorphism, it is enough to show that the morphism $\mathcal{B}_{C} \rightarrow \mathcal{T}_{C}$ from the stack of $B$-bundles on $C$ to the stack of $T$-bundles on $C$ is smooth. As usual, the obstruction to smoothness lies in a group of the form $H^{2}(C, \operatorname{ad} \alpha)$, where $\alpha \rightarrow C$ is a principal bundle under a unipotent group, so vanishes.

We shall see that 4.15 is enough to show that $s$ is representable over some neighbourhood of the locus of regular or subregular unstable $G$-bundles.

The objects of Drinfeld's compactification $\widetilde{\mathcal{G}}_{\mathcal{E}}^{D}$ are described as follows; we refer to [BG, section 1] for the details of what is described in the next paragraph.

Fix an elliptic curve $E$ and a $G$-bundle $\Xi \rightarrow E$, with $q: \mathcal{F}=\mathcal{F}_{\Xi}=\Xi \times{ }^{G} F \rightarrow$ $E$ the associated $F$-bundle. For every dominant weight $\varpi$ there is a line bundle $L_{\varpi}$ on $\mathcal{F}$ and vector bundle $V_{\varpi}=q_{*} L_{\varpi}$ on $E$. A reduction of $\Xi$ to a $B$-bundle is a line sub-bundle $M_{\varpi}$ of $V_{\varpi}$ (the line generated by a vector of highest weight) such that the set of all subsheaves $M_{\varpi} \hookrightarrow V_{\varpi}$, as $\varpi$ ranges over all dominant weights, satisfies the Plücker relations. An object of $\widetilde{\mathcal{G}}_{\mathcal{E}}^{D}$ consists of a $G$-bundle $\Xi$ over an elliptic curve $E$ and a collection of subsheaves $\left\{M_{\varpi} \hookrightarrow V_{\varpi}\right\}_{\varpi}$ where $M_{\varpi}$ is invertible, but not necessarily a sub-bundle, that satisfies the Plücker relations; we also demand that the associated $T$-bundle should have degree 0 . (This $T$ bundle is constructed as follows: objects of $\widetilde{\mathcal{G}}_{\mathcal{E}}^{D}$ are identified with sections of the $\bar{F}$-bundle $\overline{\mathcal{F}}=\Xi \times^{G} \bar{F} \rightarrow E$ that meet the open subscheme $\mathcal{F}$ of $\overline{\mathcal{F}}$. The open embedding $F \hookrightarrow \bar{F}$ induces an isomorphism $\operatorname{Pic}_{\bar{F}}^{G} \rightarrow \operatorname{Pic}_{F}^{G}$, so there is a natural $T$-bundle associated to $\overline{\mathcal{F}}$; this is to be of degree 0 .)

The projection $\widetilde{\mathcal{G}}_{\mathcal{E}}^{D} \rightarrow \mathcal{G}_{\mathcal{E}}$ is projective, while $\widetilde{\mathcal{G}}^{K M} \rightarrow \mathcal{G}$ is also proper but can have non-trivial, but finite, relative automorphism groups.

Proposition 4.2 There is a natural morphism $\widetilde{\mathcal{G}}_{\mathcal{E}} \rightarrow \widetilde{\mathcal{G}}_{\mathcal{E}}^{D}$ relative to $\mathcal{G}_{\mathcal{E}}$.

PROOF: An object of $\widetilde{\mathcal{G}}_{\mathcal{E}}$ gives sub-line bundles $M_{\varpi}^{\prime}$ of $V_{\varpi}$ on a pre-stable curve $C$ where each $M_{\varpi}^{\prime}$ has degree 0; pushing these sheaves $M_{\varpi}$ forward to $E$ gives an object of $\widetilde{\mathcal{G}}_{\mathcal{E}}^{D}$. 


\section{Some deformations}

and the stack $\widetilde{\mathcal{G}}_{\mathcal{E}}^{+}$Assume that $G$ is a split and simply connected reductive group of type $E_{l}$, where $l=5,6,7$ or 8 , and that $T \subset G$ is a copy of $\operatorname{Hom}\left(\mathrm{Pic}_{F}^{G}, \mathbb{G}_{m}\right)$, a maximal torus in $G$. Fix also a Borel subgroup $B$ containing $T$. We shall assume, as we may, that these data are all given over Spec $\mathbb{Z}$. For $E_{6}, E_{7}$ and $E_{8}$ we shall number the nodes of Dynkin diagrams as in Bourkaki's planches: the branch node is numbered 4 and the node adjacent to it on the long arm (or one of the two longer arms in the case of $E_{6}$ ) is numbered 5 . For $E_{5}=D_{5}$ we also number the branch node by 4 , but 5 will refer to a node on one of the short arms. Here is the diagram for $E_{8}$.

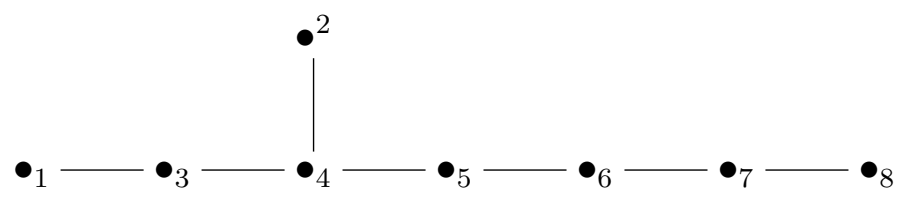

Recall, from [FM], the construction of miniversal deformation spaces for certain unstable $G$-bundles over an elliptic curve $E \rightarrow S$, where $S$ is some scheme. Suppose that $P \supset B$ is a maximal parabolic subgroup, with a Levi factor $\Lambda=$ $P \cap P^{-}$, where $P^{-} \supset B^{-}$are opposite to the pair $P \supset B$. Fix a $\Lambda$-bundle $\lambda \rightarrow E$ such that

(i) $\lambda$ is semi-stable,

(ii) $\Xi=\lambda \times{ }^{\Lambda} G$ is an unstable $G$-bundle and

(iii) $\lambda$ is regular, in the sense that for every geometric point of $S$ the automorphism group of $\lambda$ is of minimal dimension amongst all $\Lambda$-bundles that satisfy $(i)$ and $(i i)$.

Then consider the stack $\mathcal{H}$ whose objects are triples $\left(\lambda, \mathcal{P}^{-}, \phi\right)$, where $\mathcal{P}^{-}$is a $P^{-}$-bundle over $E$ and $\phi$ is an isomorphism $\phi: \mathcal{P}^{-} / U^{-} \rightarrow \lambda$, where $U^{-}$is the unipotent radical of $P^{-}$. The forgetful map $\mathcal{H} \rightarrow \Lambda_{\mathcal{E}, \text { reg }}^{\text {ss }}$, where $\Lambda_{\mathcal{E} \text {,reg }}^{\text {ss }}$ is the stack of regular semi-stable $\Lambda$-bundles over $E$, is represented by an affine space bundle over $\Lambda_{\mathcal{E}, \text { reg }}^{s s}$; the fibre over $\lambda$ is the non-abelian cohomology set $H^{1}\left(E, \underline{U}^{-}\right)$, where $\underline{U}^{-}$is the principal $U^{-}$-bundle $\lambda \times{ }^{\Lambda} U^{-}$. However, it is shown in [FM], section 4 , that this set is naturally an affine space isomorphic to the cohomology vector space $H^{1}\left(E, \underline{\mathfrak{u}}^{-}\right)$, where $\underline{\mathfrak{u}}^{-}$is the corresponding bundle of Lie algebras. (In loc. cit. the base is Spec $\mathbb{C}$ but their argument is valid over any base. So if $E$ is defined over a base scheme $S$ then $H^{1}\left(E, \underline{U}^{-}\right)$is an affine space bundle over $S$.) In particular, $\mathcal{H}$ is algebraic and smooth.

Consider the morphism $\rho: \mathcal{H} \rightarrow \mathcal{G}_{\mathcal{E}}$ given by $\rho\left(\mathcal{P}^{-}\right)=\mathcal{P}^{-} \times{ }^{P^{-}} G$. Note that there is an action of the centre $\zeta(\Lambda)$ on $\mathcal{H}$ that covers the trivial action on $\mathcal{G}$, coming from the inclusion $\zeta(\Lambda) \hookrightarrow \mathcal{P}^{-}$. The fixed locus of this action is the stack of triples $\left(\lambda, \lambda \times{ }^{\Lambda} P^{-}, \phi_{\text {can }}\right)$, which is a copy of $\Lambda_{\text {reg }}^{\text {ss }}$. That is, the affine bundle $\mathcal{H} \rightarrow \mathcal{L}_{\text {reg }}^{\text {ss }}$ has a $\mathbb{G}_{m}$-action and a section consisting of fixed points for the $\mathbb{G}_{m}$-action. 
Now suppose that we start with an unstable $G$-bundle $\xi$, defined over some algebraically closed field $k$, that is either regular or subregular. Then, according to [HS2], Theorem $5.12, \xi$ is isomorphic, up to translation by a $k$-point of $E$, to the bundle $\Xi_{r}=\lambda_{r} \times{ }^{\Lambda_{r}} G$ where $r=4$ if $\xi$ is regular and $r=5$ if $\xi$ is subregular, $\lambda_{r}$ is a semi-stable $\Lambda_{r}$-bundle and $\Lambda_{r}$ is a Levi subgroup of a maximal parabolic subgroup $P_{r}$ that is associated to the node numbered $r$ From its description, the group $\Lambda_{r}$ is defined over $\mathbb{Z}$.

Lemma 5.1 The bundle $\lambda_{r}$ is defined over $\mathcal{M}_{\text {Ell }}$.

PROOF: When $r=4$ this follows from the description of $\lambda_{r}$ on pp. 375-376 of [HS2] and the fact that $\mathcal{E}$ has a natural line bundle of degree 1, corresponding to the origin $0_{\mathcal{E}}$.

When $r=5$ there is a similar description: $\lambda_{5}$ is determined by a $G L_{q} \times G L_{s}$ bundle $\left(\eta_{q}, \eta_{s}\right)$ with $\operatorname{deg} \operatorname{det} \eta_{s}=1$ and $\operatorname{det} \eta_{q} \cong \operatorname{det} \eta_{s}^{\otimes 2}$, where $(q, s)=(5, l-4)$.

Therefore there is a versal deformation space $\mathcal{Z}^{\dagger}$ for $\xi$ that is an affine space bundle, on which $\mathbb{G}_{m}$ acts with strictly positive weights, over $\operatorname{Pic}^{1}(\mathcal{E})$. The fixed locus of the $\mathbb{G}_{m}$-action is a section and we can projectivize to form $\mathbb{P}\left(\mathcal{Z}^{\dagger}\right) \rightarrow$ $\operatorname{Pic}^{1}(\mathcal{E})$.

Let $\mathcal{Z}^{\dagger s s}$ be the semistable locus in $\mathcal{Z}^{\dagger}$; this is open, and its complement has codimension $\geq 2$. There is a classifying morphism $\gamma: \mathcal{Z}^{\dagger s s} \rightarrow[\mathcal{Y} / W]$.

Consider the induced morphism $\phi: \mathcal{Z}^{\dagger s s} \rightarrow[\mathcal{Y} / W] \times_{\mathcal{M}_{E l l}} \operatorname{Pic}^{1}(\mathcal{E})$. This morphism is constant on $\mathbb{G}_{m}$-orbits and therefore factors through a morphism $\psi:\left[\mathcal{Z}^{\dagger s s} / \mathbb{G}_{m}\right] \rightarrow[\mathcal{Y} / W] \times \mathcal{M}_{E l l} \operatorname{Pic}^{1}(\mathcal{E})$

Note that $\left[\mathcal{Z}^{\dagger s s} / \mathbb{G}_{m}\right]$ is an open substack of the weighted projective space bundle $\mathbb{P}\left(\mathcal{Z}^{\dagger}\right)$ over $[\mathcal{Y} / W] \times{ }_{\mathcal{M}_{E l l}} \operatorname{Pic}^{1}(\mathcal{E})$ and its complement is of codimension at least 2 .

For any line bundle $\mathcal{L}$ on $[\mathcal{Y} / W] \times{ }_{\mathcal{M}_{E l l}} \operatorname{Pic}^{1}(\mathcal{E})$ that is ample relative to $\operatorname{Pic}^{1}(\mathcal{E})$, let $\widehat{\mathcal{L}} \rightarrow \operatorname{Pic}^{1}(\mathcal{E})$ be the family of corresponding cones over $[\mathcal{Y} / W]$ obtained by contracting the 0 -section $0_{\mathcal{L}}$ of the total space of $\mathcal{L}$. So there is a commutative diagram

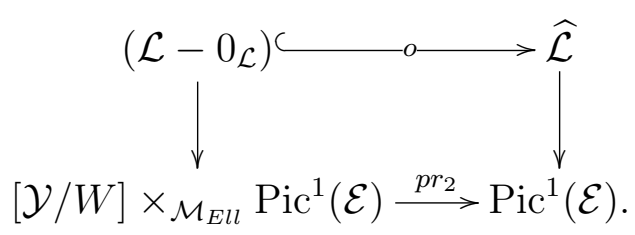

Lemma 5.2 Suppose that $\mathcal{L}_{1}$ is a line bundle on $[\mathcal{Y} / W] \times \mathcal{M}_{E l l} \operatorname{Pic}^{1}(\mathcal{E})$ that is ample relative to $\operatorname{Pic}^{1}(\mathcal{E})$. Then there is a line bundle $\mathcal{A}$ on $\operatorname{Pic}^{1}(\mathcal{E})$ such that, if $\mathcal{L}=\mathcal{L}_{1} \otimes p r_{2}^{*} \mathcal{A}$, then $\phi$ lifts to $\widetilde{\phi}: \mathcal{Z}^{\dagger s s} \rightarrow \mathcal{L}-0_{\mathcal{L}}$.

PROOF: Write $\mathcal{M}^{0}=\psi^{*} \mathcal{L}_{1}$. Then, after replacing $\mathcal{L}_{1}$ by $\mathcal{L}$ as in the statement of the lemma, we have $\mathcal{M}^{0}=\left.\mathcal{O}_{\mathbb{P}\left(\mathcal{Z}^{\dagger}\right)}(n)\right|_{\left[\mathcal{Z}^{\dagger s s} / \mathbb{G}_{m}\right]}$ for some $n \geq 1$. 
Write $\mathcal{M}=\mathcal{O}_{\mathbb{P}\left(\mathcal{Z}^{\dagger}\right)}(n)$. Then $\mathcal{M}^{0}-0_{\mathcal{M}^{0}}=\mathcal{Z}^{\dagger s s} / \mu_{n}$ and there is a commutative diagram

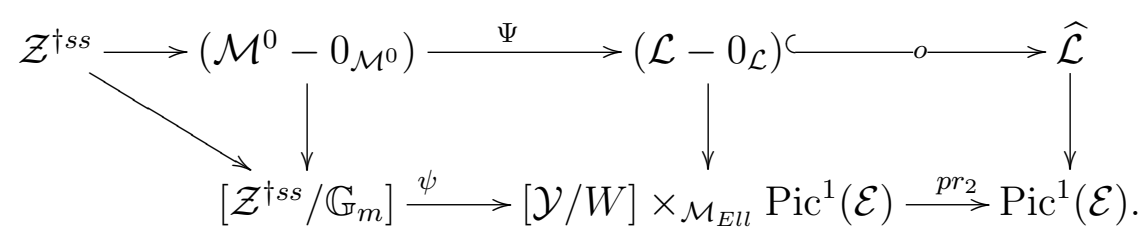

Composing the arrows in the top row gives the result.

Lemma 5.3 Suppose that $\mathcal{T}$ is a stack, that $\mathcal{X}$ and $\mathcal{V}$ are flat and affine over $\mathcal{T}$, that $\mathcal{X}$ is normal and that $\mathcal{U}$ is an open substack of $\mathcal{X}$ whose complement has codimension at least 2 in every fibre of $\mathcal{X} \rightarrow \mathcal{T}$. Then every morphism $f: \mathcal{U} \rightarrow \mathcal{V}$ over $\mathcal{T}$ extends uniquely to a morphism $\mathcal{X} \rightarrow \mathcal{V}$.

PROOF: Suppose that $T \rightarrow \mathcal{T}$ is a smooth cover and that $T$ is affine. Write $\mathcal{U}_{T}=\mathcal{U} \times_{\mathcal{T}} T$ etc. and we have a diagram

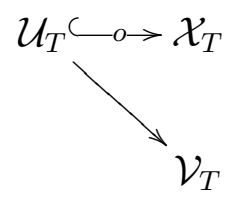

of schemes, where $\mathcal{X}_{T}$ and $\mathcal{V}_{T}$ are affine and $\mathcal{X}_{T}$ is normal. Now

$$
\begin{aligned}
\operatorname{Mor}_{S c h / T}\left(\mathcal{U}_{T}, \mathcal{V}_{T}\right) & =\operatorname{Mor}_{\text {Rings } / \Gamma\left(\mathcal{O}_{T}\right)}\left(\Gamma\left(\mathcal{O}_{\mathcal{V}_{T}}\right), \Gamma\left(\mathcal{O}_{\mathcal{U}_{T}}\right)\right) \\
& =\operatorname{Mor}_{\text {Rings } / \Gamma\left(\mathcal{O}_{T}\right)}\left(\Gamma\left(\mathcal{O}_{\mathcal{V}_{T}}\right), \Gamma\left(\mathcal{O}_{\mathcal{X}_{T}}\right)\right)=\operatorname{Mor}_{S c h / T}\left(\mathcal{X}_{T}, \mathcal{V}_{T}\right)
\end{aligned}
$$

since $\Gamma\left(\mathcal{O}_{\mathcal{U}_{T}}\right)=\Gamma\left(\mathcal{O}_{\mathcal{X}_{T}}\right)$. So the lemma is proved in the scheme-theoretical context and then follows in full via descent.

Corollary 5.4 The morphism $\gamma: \mathcal{Z}^{\dagger s s} \rightarrow[\mathcal{Y} / W] \times{ }_{\mathcal{M}_{E l l}} \operatorname{Pic}^{1}(\mathcal{E})$ lifts to a morphism $\mathcal{Z}^{\dagger} \rightarrow \widehat{\mathcal{L}}$.

PROOF: This follows from the previous two lemmas.

Looijenga [L1] considered ample $W$-linearized line bundles $\mathcal{L}$ on $\mathcal{Y}$ such that the linearization is trivial on the line $\mathcal{L}\left(0_{\mathcal{Y}}\right)$ and the polarization defined by $\mathcal{L}$ is the standard $W$-invariant quadratic form on the weight lattice $P$.

He proved that the set of such line bundles is naturally a torsor under the finite group scheme $\operatorname{Hom}(P / Q, \mathcal{E})$ and that over any complex point of $\mathcal{M}_{\text {Ell }}$ the ring of invariants $\oplus_{n \geq 0} H^{0}\left(\mathcal{Y}, \mathcal{L}^{\otimes n}\right)^{W}$ is a polynomial ring and is generated by homogeneous elements whose degrees are the coefficients of the biggest (highest) root when expressed in terms of simple roots. (Recall that we are only considering simply laced groups.) 
Proposition 5.5 There exists a line bundle on $\mathcal{Y}$ that satisfies Looijenga's hypotheses.

PROOF: Assume that $r \leq 8$ and let $\Lambda$ denote the hyperbolic lattice $\Lambda=\mathbb{Z} e_{0} \oplus$ $\oplus_{1}^{r} \mathbb{Z} e_{i}$ with $e_{0}^{2}=-1, e_{i}^{2}=1$ for $i \geq 1$ and $e_{i} \cdot e_{j}=0$ otherwise. Put $\kappa=3 e_{0}-\sum_{1}^{r} e_{i}$. Then $P \cong \Lambda / \mathbb{Z} \kappa$ and the exact sequence

$$
0 \rightarrow \mathbb{Z} \kappa \rightarrow \Lambda \rightarrow P \rightarrow 0
$$

gives an exact sequence

$$
0 \rightarrow \mathcal{Y} \rightarrow \operatorname{Hom}(\Lambda, \mathcal{E}) \rightarrow \mathcal{E} \rightarrow 0
$$

For $j=0,1, \ldots, r$ we have $\mathbb{Z} e_{j} \hookrightarrow \Lambda$, so projections $p r_{j}: H o m(\Lambda, \mathcal{E}) \rightarrow \mathcal{E}$. Define $\mathcal{A}=\mathcal{O}_{\mathcal{E}}\left(0_{\mathcal{E}}\right)$ and $\mathcal{A}_{j}=p_{j}^{*} \mathcal{A}$. Then $\mathcal{L}=\mathcal{A}_{0}^{-1} \otimes \mathcal{A}_{1} \otimes \cdots \otimes \mathcal{A}_{r}$ is the line bundle required.

That is, each regular or subregular unstable bundle $\Xi_{4}$ or $\Xi_{5}$ has a versal deformation space that is an affine bundle over $\operatorname{Pic}^{1}(\mathcal{E})$, and after turning off $\operatorname{Pic}^{1}(\mathcal{E})$ the affine space that is the fibre admits a classifying morphism to the cone $[\widehat{\mathcal{Y}} / W]$ corresponding to the line bundle $\mathcal{L}$.

Now we construct the stack $\widetilde{\mathcal{G}}_{\mathcal{E}}^{+}$. Fix the $G$-homogeneous spaces $H_{4}, H_{5}$ and $H_{4,5}$ associated to the sets of nodes $\{4\},\{5\}$ and $\{4,5\}$, so that $F \rightarrow H_{4}$ and $F \rightarrow H_{5}$ are $\mathbb{P}^{1}$-bundles, $H_{4} \rightarrow H_{4,5}$ and $H_{5} \rightarrow H_{4,5}$ are $\mathbb{P}^{2}$-bundles and $F \rightarrow H_{4,5}$ is a bundle whose fibre is the flag variety $S L_{3} / B$ of type $A_{2}$.

Given $S \rightarrow \mathcal{M}_{E l l}$, the $S$-objects of $\widetilde{\mathcal{G}}_{\mathcal{E}}^{+}$are triples $(\Xi, \sigma, \tau)$ where $\Xi$ is a $G$ bundle $\Xi \rightarrow \mathcal{E} \times \times_{\mathcal{M}_{E l l}} S, \sigma$ a stable map $\sigma: C \rightarrow \mathcal{H}_{5}=\Xi \times^{G} H_{5}$ and $\tau$ a section of $\overline{\mathcal{F}}$ such that the associated $T$-bundle is of degree 0 . Moreover, we require that under the projection $\overline{\mathcal{F}} \rightarrow \overline{\mathcal{H}}_{5}=\Xi \times^{G} \bar{H}_{5}$ the curves $\sigma$ and $\tau$ should agree over a dense open subset $U$ of $\mathcal{E} \times{ }_{\mathcal{M}_{E l l}} S$ that meets every geometric fibre of $\mathcal{E} \times \mathcal{M}_{E l l} S \rightarrow S$. It follows from the definition that $\widetilde{\mathcal{G}}_{\mathcal{E}}^{+}$is a closed substack of $\widetilde{\mathcal{G}}_{\mathcal{E}} \times{ }_{\mathcal{G}_{\mathcal{E}}} \widetilde{\mathcal{G}}_{\mathcal{E}}^{D}$.

Proposition 5.6 The morphism $\widetilde{\mathcal{G}}_{\mathcal{E}} \rightarrow \widetilde{\mathcal{G}}_{\mathcal{E}}^{D}$ factors through $\widetilde{\mathcal{G}}_{\mathcal{E}}^{+}$.

PROOF: This is an immediate consequence of Proposition 4.2 and the existence of a projection $\mathcal{F} \rightarrow \mathcal{H}_{5}$ of bundles over $\mathcal{E} \times_{\mathcal{M}_{E l l}} S$.

\section{Subregular unstable bundles and del Pezzo surfaces}

We keep the notation of Section 5. In particular, $r=4$ or 5 .

There is a decomposition

$$
\underline{\mathfrak{g}}_{r}:=\operatorname{ad} \Xi_{r}=\underline{\mathfrak{l}}_{r} \oplus \underline{\mathfrak{u}}_{r} \oplus \underline{\mathfrak{u}}_{r}^{*}
$$


of vector bundles on $\mathcal{E}$, where $\underline{\mathfrak{l}}_{r}=\operatorname{ad} \lambda_{r}$ consists of summands of degree $0, \underline{\mathfrak{u}}_{r}$ consists of summands of positive degree and $\underline{\mathfrak{l}}_{r} \oplus \underline{\mathfrak{u}}_{r}=\operatorname{ad}\left(\lambda_{r} \times{ }^{\Lambda_{r}} P_{r}\right)$.

We know that there is a miniversal deformation space $\mathcal{Z}^{\dagger}$ for $\Xi_{5}$ with a morphism $\mathcal{Z}^{\dagger} \rightarrow[\widehat{\mathcal{Y}} / W]$ that preserves $\mathbb{G}_{m}$-orbits; note that this is weaker than the statement that the morphism is $\mathbb{G}_{m}$-equivariant.

Define $\widetilde{\mathcal{Z}}^{\dagger}=\mathcal{Z}^{\dagger} \times_{\mathcal{G}_{\mathcal{E}}} \widetilde{\mathcal{G}}_{\mathcal{E}}$, with projection $\nu: \widetilde{\mathcal{Z}}^{\dagger} \rightarrow \mathcal{Z}^{\dagger}$. By restricting to $\mathcal{G}_{\mathcal{E}}^{s s}$ we see that $\nu$ is generically finite and that $\operatorname{deg} \nu=\# W$. The existence of the morphisms $\pi: \widetilde{\mathcal{G}}_{\mathcal{E}} \rightarrow \mathcal{Y}$ and $\mathcal{Z}^{\dagger} \rightarrow[\widehat{\mathcal{Y}} / W]$ gives a square

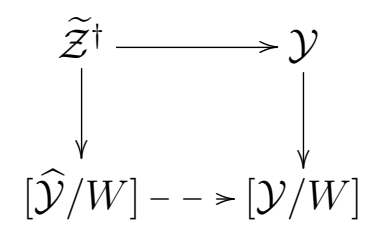

where the lower broken arrow is a rational map, the projection of a cone from its vertex to its base. On the open substack $\nu^{-1}\left(\mathcal{Z}^{\dagger s s}\right)$ this square is commutative, and so there is a factorization through the line bundle $\mathcal{L} \rightarrow \mathcal{Y}$ corresponding to the cone $\widehat{\mathcal{Y}}$ :

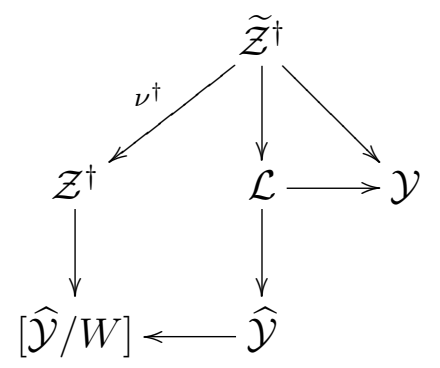

where $\operatorname{deg} \nu^{\dagger}=\# W$.

The next lemma is a slight strengthening of 4.14 .

Lemma 6.1 $\widetilde{\mathcal{Z}}^{\dagger} \rightarrow \operatorname{Pic}^{1}(\mathcal{E}) \times_{\mathcal{M}_{E l l}} \mathcal{C}_{\text {pre }} \times_{\mathcal{M}_{E l l}} \mathcal{Y}$ is smooth.

PROOF: $\widetilde{\mathcal{Z}}^{\dagger}$ classifies $B$-bundles over pre-stable curves. The obstruction to smoothness lies in an extension of groups each of the form $H^{2}(C, \mathcal{U})$ where $C$ is a pre-stable curve and $\mathcal{U}$ is a commutative unipotent group scheme over $C$. Since $C$ is 1-dimensional this obstruction vanishes.

Now restrict to the point $\left[0_{\mathcal{E}}\right]$ of $\operatorname{Pic}^{1}(\mathcal{E})$; that is, turn off $\operatorname{Pic}^{1}(\mathcal{E})$. Let $\mathcal{Z}$ and $\widetilde{\mathcal{Z}}$ denote the resulting spaces; they fit into a commutative diagram

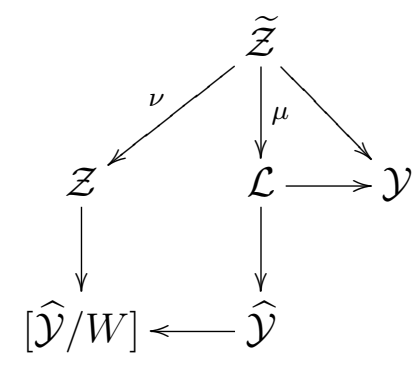


where also $\operatorname{deg} \nu=\# W$. Note that, by Lemma $6.1, \widetilde{\mathcal{Z}} \rightarrow \mathcal{C}_{\mathcal{M}_{\text {Ell }}} \mathcal{Y}$ is smooth and the unstable locus $\widetilde{\mathcal{Z}}^{u}$ in $\widetilde{\mathcal{Z}}$ (defined as the inverse image $\nu^{-1}\left(\mathcal{Z}^{u}\right)$ of the unstable locus $\mathcal{Z}^{u}$ in $\mathcal{Z}$ ) equals $\mu^{-1}\left(0_{\mathcal{L}}\right)$, where $0_{\mathcal{L}}$ is the zero-section of $\mathcal{L}$. Moreover, $\widetilde{\mathcal{Z}}$ is smooth over the stack $\mathcal{C}_{\text {pre }}$ of elliptic curves with rational tails, and $\widetilde{\mathcal{Z}}^{u}$ is the inverse image of the discriminant divisor $\Delta_{\mathcal{C}_{\text {pre }}}$ in $\mathcal{C}_{\text {pre }}$. So $\mu^{-1}\left(0_{\mathcal{L}}\right)$ has normal crossings. However, we do not yet know that $\mu^{-1}\left(0_{\mathcal{L}}\right)$ is reduced; this will be proved in Theorem 6.7 below.

By construction, $\mathcal{Z}^{u}$ is the inverse image of the vertex of the cone $[\widehat{\mathcal{Y}} / W]$, so that $\mathcal{Z}^{u}$ is a surface, relative to $\mathcal{M}_{E l l}$, with two strata: $\mathcal{Z}^{u}=\left\{\left[\Xi_{5}\right]\right\} \cup\left(\mathcal{Z}^{u}-\left\{\left[\Xi_{5}\right]\right\}\right)$; the points of $\mathcal{Z}^{u}-\left\{\left[\Xi_{5}\right]\right\}$ correspond to regular unstable bundles, all of which are isomorphic to $\Xi_{4}$.

The next result, giving a version of Steinberg's cross-section theorem which underlies the classical BGSS construction, is due to Friedman and Morgan [FM], Theorem 5.1.1; see also [Brue]. From it, they get a new proof of Looijenga's result $[\mathrm{L} 1]$, that $[\widehat{Y} / W]$ is an affine space. They all assume that the ground field is $\mathbb{C}$ but specialization extends the result to all characteristics, as we show.

Theorem 6.2 (1) $[F M]$ For any geometric point $\eta$ in $\mathcal{Z}^{u}-\left\{\left[\Xi_{5}\right]\right\}$ there is a section of the morphism $\mathcal{Z} \rightarrow[\widehat{\mathcal{Y}} / W]$ through $\eta$.

(2) $[L 1][\widehat{\mathcal{Y}} / W]$ is isomorphic to an affine space bundle over $\mathcal{M}_{\text {Ell }}$ whose fibre is $\mathbb{A}^{l+1}$.

PROOF: Friedman and Morgan construct, for the regular unstable bundle $\Xi_{4}$, a chart $V$ that is isomorphic to $H^{1}\left(\mathcal{E}\right.$, ad $\left.\Xi_{4}\right)$, a vector bundle over $\mathcal{M}_{E l l}$. Their main result is that the classifying morphism $\hat{h}: V \rightarrow[\widehat{\mathcal{Y}} / W]$, which preserves $\mathbb{G}_{m}$-orbits, induces a morphism $h: \mathbb{P}(V) \rightarrow[\mathcal{Y} / W]$ defied over $\mathcal{M}_{\text {Ell }}$ which is an isomorphism over $\mathcal{M}_{E l l, \mathbb{Q}}=\mathcal{M}_{E l l} \otimes_{\mathbb{Z}} \mathbb{Q}$.

Now the formation of geometric quotients does not always commute with reduction modulo $p$, so we know only that $[\mathcal{Y} / W]$ is normal and the geometric fibres of $[\mathcal{Y} / W] \rightarrow \mathcal{M}_{E l l}$ are generically reduced (on the locus where $W$ acts freely). However, $h: \mathbb{P}(V) \rightarrow[\mathcal{Y} / W]$ provides a simultaneous normalization of the fibres of the family $[\mathcal{Y} / W] \rightarrow \mathcal{M}_{E l l}$ (without the requirement for any base change) and it follows that $h$ is an isomorphism.

Moreover, the weights of the $\mathbb{G}_{m}$-action on $V$ and on $[\widehat{Y} / W]$ are the same on both sides, so that $h^{*} L \cong \mathcal{O}_{\mathbb{P}(V)}(1)$ and $\hat{h}$ is an isomorphism.

By specialization, it follows that $h$ is an isomorphism in characteristic $p$, and then we deduce similarly that $\hat{h}$ is an isomorphism in characteristic $p$.

By the openness of versality, $\mathcal{Z}$ is also a chart for $\eta$; that is, $\mathcal{Z} \rightarrow \mathcal{G}_{\mathcal{E}}$ is smooth at $\eta$, so an appropriate slice of $\mathcal{Z}$ through $\eta$ gives the section required.

Now fix a geometric point of $\mathcal{M}_{E l l}$, corresponding to an elliptic curve $E$ over an algebraically closed field $k$. Fix the origin $0_{Y}$ of $Y=\operatorname{Hom}(P, E)$ and the copy of $\mathbb{A}_{k}^{1}$ that is the line in $L=\left.\mathcal{L}\right|_{Y}$ lying over $0_{Y}$. Let $\widetilde{X} \rightarrow \mathbb{A}_{k}^{1}$ denote the restriction of $\widetilde{Z}$ to this line. That is, $\widetilde{X}$ is the fibre over $0_{Y}$ of the smooth morphism $\widetilde{Z} \rightarrow Y$, 
so that $\widetilde{X}$ is smooth, of dimension $\operatorname{dim} \widetilde{X}=\operatorname{dim} \widetilde{Z}-\operatorname{dim} Y=l+3-l=3$, and the zero fibre $\widetilde{X}_{0}$ of $\widetilde{X} \rightarrow \mathbb{A}_{k}^{1}$ has normal crossings.

Now $\mathbb{A}_{k}^{1}$ maps isomorphically to its image $\Gamma$ in $[\widehat{Y} / W]$, since $W$ acts trivially on this line $\mathbb{A}_{k}^{1}$, and so there is a commutative diagram

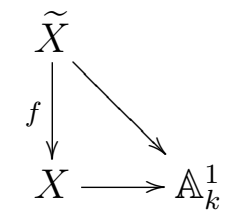

where $X$ is the image of $\widetilde{X}$ in $Z$ and $\widetilde{X} \rightarrow X$ is proper and birational. Moreover, this diagram is $\mathbb{G}_{m}$-equivariant, since $f$ takes $\mathbb{G}_{m}$-orbits to $\mathbb{G}_{m}$-orbits and is birational, $0 \in \mathbb{A}_{k}^{1}$ is a fixed point and $\mathbb{A}_{k}^{1}-\{0\}$ is homogeneous (but not necessarily a torsor; this will be proved later, in Lemma 6.23) under the $\mathbb{G}_{m}$-action.

Lemma 6.3 $X$ has local complete intersection (LCI) singularities and $X_{0}$ is a normal surface with a unique singular point, namely the point $[\xi]$ corresponding to $\xi$.

PROOF: $\quad X$ is the inverse image in $Z$ of the line $\Gamma$ in $[\widehat{Y} / W]$. Since $[\widehat{Y} / W] \cong \mathbb{A}_{k}^{l+1}$ and $Z$ is smooth, the singularities of $X$ are LCI. That $[\xi]$ is isolated follows from the fact that for the exceptional groups there is (up to choosing a point on $\operatorname{Pic}^{1}(E)$, which amounts to translating $\xi$ by a point on $E$ ) just one subregular unstable bundle and the subregular unstable locus has codimension 2 in the unstable locus.

Write $\mathcal{F}_{r}=\Xi_{r} \times{ }^{G} F$, the associated $F$-bundle over $E$. We know, from the computation of Helmke and Slodowy, that $\operatorname{dim} H^{0}\left(E, \underline{\mathfrak{g}}_{5}\right)=l+4$.

Proposition 6.4 Suppose that $\sigma$ is a section of $\mathcal{F}_{r}$ that gives a negative cocharacter $[\sigma]$.

(1) If $r=4$, then $[\sigma]=-\alpha_{4}^{\vee}$.

(2) If $r=5$, then $[\sigma]=-\alpha_{4}^{\vee}$ or $-\alpha_{5}^{\vee}$ or $-\alpha_{4}^{\vee}-\alpha_{5}^{\vee}$.

PROOF: A section $\sigma$ of $\mathcal{F}_{r}$ corresponds to a $B$-bundle $\mathcal{B}_{\sigma} \rightarrow E$ such that the induced bundle $\mathcal{B}_{\sigma} \times{ }^{B} G$ equals $\Xi_{r}$.

Suppose $\mathfrak{u}_{\Sigma}$ is an ideal in Lie $B, \Sigma$ the corresponding subset of the set of positive roots, $\underline{\mathfrak{u}}_{\Sigma}=\mathfrak{u}_{\Sigma} \times{ }^{B} \mathcal{B}$ the bundle attached to this by $\mathcal{B}$ and $2 \rho_{\Sigma}=\sum_{\alpha \in \Sigma} \alpha$. Then, writing $[\sigma]=-\sum_{i \in I} s_{i} \alpha_{i}^{\vee}, s_{i} \geq 0$, we have

$$
\operatorname{deg} \underline{\mathfrak{u}}_{\Sigma}=\left(-[\sigma], 2 \rho_{\Sigma}\right)=\left(\sum s_{i} \alpha_{i}^{\vee}, 2 \rho_{\Sigma}\right)
$$

As $\underline{\mathfrak{u}}_{\Sigma}$ is a sub-bundle of $\underline{\mathfrak{g}}$, we have $\operatorname{dim} H^{0}\left(E, \underline{\mathfrak{g}}_{r}\right) \geq \operatorname{dim} H^{0}\left(E, \underline{\mathfrak{u}}_{\Sigma}\right) \geq \operatorname{deg} \underline{\mathfrak{u}}_{\Sigma}$. 
Lemma $6.5 \operatorname{dim} H^{0}\left(E, \underline{\mathfrak{g}}_{r}\right)>\operatorname{deg} \underline{\mathfrak{u}}_{\Sigma}$.

PROOF: Suppose otherwise. Then $\operatorname{deg} \underline{\mathfrak{u}}_{\Sigma}=\operatorname{dim} H^{0}\left(E, \underline{\mathfrak{u}}_{\Sigma}\right)$, and so the only possible indecomposable summands of the vector bundle $\underline{\mathfrak{u}}_{\Sigma}$ with slope zero must have non-trivial determinant; the remaining summands have strictly positive slope.

Next, consider the canonical reduction of $\underline{\mathfrak{g}}_{r}$. This is $\underline{\mathfrak{g}}_{r}=\underline{\mathfrak{l}}_{r}+\underline{\mathfrak{n}}^{+}+\underline{\mathfrak{n}}^{-}$, where $\underline{\mathfrak{l}}_{r}=\zeta\left(\underline{\mathfrak{l}}_{r}\right)+\left[\underline{\mathfrak{l}}_{r}, \underline{\mathfrak{l}}_{r}\right]$ is the decomposition of $\underline{\mathfrak{l}}_{r}$ as the direct sum of its centre and the bundle of its derived subalgebra, $\zeta\left(\underline{\mathfrak{l}}_{r}\right)$ is a non-zero direct sum of trivial line bundles, $\left[\underline{\mathfrak{l}}_{r}, \underline{\mathfrak{l}}_{r}\right]$ is a direct sum of semi-stable bundles of degree 0 , and $\underline{\mathfrak{n}}^{+}$(resp. $\underline{\mathfrak{n}}^{-}$) is a direct sum of vector bundles of strictly positive (resp. negative) slopes. Then $\underline{\mathfrak{u}}_{\Sigma}$ embeds into $\left[\underline{\mathfrak{l}}_{r}, \underline{\mathfrak{l}}_{r}\right]+\underline{\mathfrak{n}}^{+}$, and hence $\operatorname{dim} H^{0}\left(E, \underline{\mathfrak{u}}_{\Sigma}\right) \leq$ $\left.\operatorname{dim} H^{0}\left(E, \underline{\mathfrak{l}}_{r}, \underline{\mathfrak{l}}_{r}\right]+\underline{\mathfrak{n}}^{+}\right)<\operatorname{dim} H^{0}\left(E, \underline{\mathfrak{g}}_{r}\right)$.

Now let $J$ be a subset of the simple roots, and take $\Sigma_{J}$ to be the roots of the unipotent radical of the standard parabolic $P_{J}$ generated by $B$ and the negative simple roots $-\alpha$ for $\alpha \notin J$, and write $\rho_{J}$ for $\rho_{\Sigma_{j}}, \underline{\mathfrak{u}}_{J}$ for $\underline{\mathfrak{u}}_{\Sigma_{J}}$.

Suppose that $\alpha$ is simple and $\alpha \notin J$. Let $\mathfrak{s}_{\alpha}$ denote the reflection in $\alpha$. Then $\mathfrak{s}_{\alpha}\left(\Sigma_{J}\right)=\Sigma_{J}$ and $\mathfrak{s}_{\alpha}\left(\alpha^{\vee}\right)=-\alpha^{\vee}$, so $\left(\alpha^{\vee}, \rho_{J}\right)=0$, and hence $2 \rho_{J}=\sum_{j \in J} m_{j} \varpi_{j}$ for some integers $m_{j}$ depending on $J$. In particular, $2 \rho_{\{j\}}=m_{j} \varpi_{j}$ and $2 \rho_{\{j, k\}}=$ $a_{j} \varpi_{j}+a_{k} \varpi_{k}$ for some integers $m_{j}, a_{j}, a_{k}$. Evaluating on $\varpi_{j}^{\vee}$, we get $m_{j}\left(\varpi_{j}^{\vee}, \varpi_{j}\right)=$ $\left(\varpi_{j}^{\vee}, 2 \rho_{\{j\}}\right)=\left(\varpi_{j}^{\vee}, 2 \rho\right)$, and so the numbers $m_{j}$ can be read off from Bourbaki's planches; the vector $m=\left(m_{1}, \ldots, m_{l}\right)$ is given as follows, and $\operatorname{dim} H^{0}\left(E, \underline{\mathfrak{g}}_{r}\right)>$ $\operatorname{deg} \underline{\mathfrak{u}}_{\{j\}}=s_{j} m_{j}$ for all $j$.

$$
\begin{aligned}
& E_{8}: m=(23,17,13,9,11,14,19,29) \\
& E_{7}: m=(17,14,11,8,10,13,18) \\
& E_{6}: m=(12,11,9,7,9,12) \\
& D_{5}: m=(8,7,8,6,8) .
\end{aligned}
$$

(1): $r=4$. Then $l+2=\operatorname{dim} H^{0}\left(E, \underline{g}_{4}\right), s_{j}=0$ if $j \neq 4$, and $s_{4} \leq 1$. But $[\sigma]<0$, so $s_{4}>0$ and then $s_{4}=1$. This proves (1) of the Proposition.

(2): $r=5 . \Xi_{5}$ is induced from a semi-stable $\Lambda_{5}$-bundle [HS2], and $l+4=$ $\operatorname{dim} H^{0}\left(E, \mathfrak{g}_{5}\right)$, so again $s_{j} \leq 1$.

To finish, we need to show that if $j \neq 4$ or 5 , then $s_{j}=0$. If $G=E_{7}$ or $E_{8}$, this follows from $l+4=\operatorname{dim} H^{0}(E, \underline{\mathfrak{g}})>\operatorname{dim} H^{0}\left(E, \underline{\mathfrak{u}}_{\{j\}}\right)=s_{j} m_{j}$ and the values given above for the vector $m$. For $G=E_{6}$ or $D_{5}$ we can (eschewing science) calculate $\operatorname{deg} \underline{\mathfrak{u}}_{\{5, j\}}$ case by case, as necessary. If $G=E_{6}$ and $s_{3}=1$, we get $\operatorname{deg} \underline{\mathfrak{u}}_{\{5, j\}}=10=l+4$, while for $G=D_{5}$ we get $\operatorname{deg} \underline{\mathfrak{u}}_{\{5, j\}}=11,9,10$ when $j=1,2,3$, which furnishes a contradiction and thereby concludes the proof of the Proposition.

We remark that a uniform treatment of all the combinatorics in this paper will be found in [GS].

Corollary 6.6 The morphism $p: \widetilde{\mathcal{G}}_{\mathcal{E}} \rightarrow \mathcal{G}_{\mathcal{E}}$ is representable over a neighbourhood of $\Xi_{5}$ that contains $\mathcal{G}_{\mathcal{E}}^{s s}$. 
PROOF: From Proposition 6.4, any rational component of a stable map $C \rightarrow \mathcal{F}_{5}$ maps birationally to its image if it is not contracted to a point. So the map $C \rightarrow \mathcal{F}_{5}$ has no automorphisms.

The next result is the crux of our paper. It is this that shows how exceptional groups lead naturally to weak del Pezzo surfaces. The strategy of the proof is to show first, by considering which components can meet, that the only triple points on the closed fibre $\widetilde{X}_{0}$ lie on certain copies $Q$ of $\mathbb{P}^{1} \times \mathbb{P}^{1}$ and then to compute intersection numbers in as many ways as possible and use the adjunction formula as much as possible in order to derive the full result.

Note that, although the surface $Q$ that appears might appear to be annoying, its presence enables us to recognize the other surface $D_{1}$ as a weak del Pezzo surface, because the intersection $D_{1} \cap Q$ is a line on $D_{1}$ : if $Q$, and so that line, were missing then our argument would only show that $D_{1}$ is a surface whose first Chern class has a sign (modulo (-2)-curves). In the course of the proof we shall see that there is a smooth birational contraction where $Q$ is contracted onto that line.

\section{Theorem 6.7}

(1) The fibre $\tilde{X}_{0}$ is semi-stable. That is, it is a reduced union of smooth surfaces and it has normal crossings.

(2) $\widetilde{X}_{0}$ consists of three components: $\widetilde{X}_{0}=D_{0}+D_{1}+Q$, where $D_{0}$ is the strict transform of the affine surface $X_{0}, Q$ is a copy of $\mathbb{P}^{1} \times \mathbb{P}^{1}$, the normal bundle $\mathcal{N}_{Q / \tilde{X}}$ is isomorphic to $\mathcal{O}(-1,-1)$ and $D_{1}$ is a weak del Pezzo surface.

(3) Set $D_{0} \cap D_{1}=A$. Then $A$ is isomorphic to $E$ and $A$ is an anticanonical divisor on both $D_{0}$ and on $D_{1}$.

(4) $Q \cap D_{0}$ is a fibre $\beta$ of one ruling on $Q, Q \cap D_{1}$ is a fibre $\gamma$ of the other ruling, $\gamma$ is the unique $(-1)$-curve on $D_{1}$ and $\beta$ is a $(-1)$-curve on $D_{0}$.

(5) There is a number $t$ with $0 \leq t \leq l-1$ that depends only upon $G$ such that the dual graph of the exceptional locus of the contraction $D_{0} \rightarrow X_{0}$ is a chain

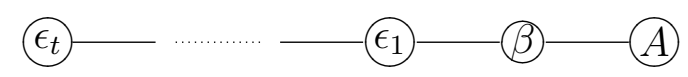

of transverse curves where each $\epsilon_{i}$ is a (-2)-curve.

(6) There is a birational map $\widetilde{X}-\rightarrow X^{-}$, where $X^{-}$is smooth and $X^{-} \rightarrow \mathbb{A}^{1}$ is also semi-stable, which is constructed by first contracting $Q$ onto $\gamma$ and then successively flopping the strict transforms of $\epsilon_{1}, \ldots, \epsilon_{t}$.

(7) $X_{0}^{-}=D_{0}^{-}+D_{1}^{-}$where $D_{0}^{-}$is the minimal resolution of $X_{0}, D_{1}^{-}$is a weak del Pezzo surface of degree $9-l$ and $D_{1}^{-}$contains a configuration $\Gamma_{0}$ of $(-2)$-curves of type $G$.

Remark: We do not need to know the value of $t$, since the curves $\beta$, $\epsilon_{i}$ will be flopped to give a family of del Pezzo surfaces of the correct degree. This issue will be addressed in Davis' thesis [D]. 
PROOF: The points $x$ of $\widetilde{X}_{0}$ correspond to configurations $C=\sigma+\phi$, where $\sigma$ is a section of either $\xi \times^{G} F$ or $\eta \times^{G} F$, where $\eta$ is regular unstable. That is, $\eta \in X_{0}-\{\xi\}$.

Since $X_{0}$ is reduced, by Theorem 6.2, it follows from Proposition 6.4 that

$$
\tilde{X}_{0}=D_{0}+\sum_{i=1}^{r} m_{i} D_{i}+\sum_{j=1}^{s} n_{j} Q_{j}+\sum r_{k} F_{k}
$$

where $D_{0}$ is the strict transform of $X_{0}$ and the other components are projective. Moreover, points in the interior of the various components correspond to configurations $\sigma+\phi$ where $\sigma$ is a copy of $E$ and the class of $\phi$ is given as follows:

(1) $D_{0}:[\phi]=\alpha_{4}^{\vee}$ or $\alpha_{5}^{\vee}$;

(2) $D_{i}^{o}$ for $i \geq 1:[\phi]=\alpha_{5}^{\vee}$;

(3) $F_{k}^{o}:[\phi]=\alpha_{4}^{\vee}$;

(4) $Q_{j}^{o}:[\phi]=\alpha_{4}^{\vee}+\alpha_{5}^{\vee}$.

Lemma 6.8 For $j \geq 1$ the $D_{j}$ are smooth and are mutually disjoint, as are the $Q_{j}$ and the $F_{k}$.

PROOF: If two branches of the divisor $\sum_{j>0} D_{j}$ were to meet, then they would meet along a curve $\delta$ that parametrized sections $\tau$ of $\mathcal{F}_{\xi}$ with $[\tau]=-\alpha_{4}^{\vee}-\alpha_{5}^{\vee}$; more precisely, $\delta$ would parametrize stable maps whose image is $\tau+\phi_{4}+\phi_{5}$ with $\left[\phi_{i}\right]=\alpha_{i}^{\vee}$. Since $\widetilde{\mathcal{G}} \rightarrow \mathcal{C}_{\text {pre }}$ is smooth, the locus of stable maps whose image contains a curve of class $\alpha_{4}$ is of pure codimension; however, it contains $\delta$ and the surface $D_{0}$. So $\sum_{j>0} D_{j}$ is smooth.

The argument for $\sum Q_{j}$ and $\sum F_{k}$ is similar.

Lemma 6.9 $Q_{j}$ is isomorphic to $\mathbb{P}^{1} \times \mathbb{P}^{1}$.

PROOF: Every point of $Q_{j}$ corresponds to a stable map $f: C=\sigma \cup \phi \rightarrow \mathcal{F}_{\xi}$ where $\sigma \cap \phi$ consists of a single point, say $x$, and $[\phi]=\alpha_{4}^{\vee}+\alpha_{5}^{\vee}$. Therefore $Q_{j}$ is isomorphic to the variety $V$ of $(1,1)$ curves on the flag variety $H=S L_{3} / B$ of type $A_{2}$ that pass through a fixed point $v_{0}$ of $H\left(v_{0}\right.$ is also here a fixed point on $\sigma)$.

So it is enough to prove that $V \cong \mathbb{P}^{1} \times \mathbb{P}^{1}$.

Recall that $H$ is the incidence variety $\{(x, l) \mid x \in l\}$, where $x$ is a point in $\mathbb{P}^{2}$ and $l$ is a line. The projections $p: H \rightarrow \mathbb{P}^{2}, q: H \rightarrow\left(\mathbb{P}^{2}\right)^{\vee}$ map $V$ to the set of points on a fixed line and to the set of lines through a fixed point, respectively. This gives a morphism $a: V \rightarrow \mathbb{P}^{1} \times \mathbb{P}^{1}$.

Suppose that $\gamma, \delta$ are $(1,1)$ curves on $H$ with $p(\gamma)=p(\delta)=m$ and $q(\gamma)=$ $q(\delta)=n$. So $m, n$ are lines. Then $\gamma, \delta$ are curves in $p^{-1}(m)$ with equal image under $q$. Now $p^{-1}(m)$ is a copy of the Hirzebruch surface $\mathbb{F}_{1}$ and $q: \mathbb{F}_{1} \rightarrow\left(\mathbb{P}^{2}\right)^{\vee}$ exhibits $\mathbb{F}_{1}$ as the blow-up of $\left(\mathbb{P}^{2}\right)^{\vee}$ at a point. Then $\gamma=\delta$, so that $a$ is an isomorphism, as required. 
Lemma 6.10 $Q_{j} \cap\left(\widetilde{X}_{0}-Q_{j}\right)=\beta \cup \gamma$ where $\beta$, $\gamma$ are opposite rulings on $\mathbb{P}^{1} \times \mathbb{P}^{1}$. PROOF: $\quad Q_{j} \cap\left(\widetilde{X}_{0}-Q_{j}\right)$ parametrizes stable maps which are either of the form $f: C=\sigma \cup \phi_{4} \cup \phi_{5} \rightarrow \mathcal{F}_{\xi}$ or of the form $f: C=\sigma \cup \psi \cup \phi_{4} \cup \phi_{5} \rightarrow \mathcal{F}_{\xi}$ where in each case $\left[f\left(\phi_{i}\right]=\alpha_{i}^{\vee}\right.$ and $f(\psi)$ is a point. Also $\sigma \cap\left(\phi_{4} \cup \phi_{5}\right)$ is a single point, resp., $\sigma \cap\left(\psi \cup \phi_{4} \cup \phi_{5}\right)$ is a single point. There are three possibilities.

(1) $\sigma$ and $\phi_{4}$ remain fixed and $\phi_{5}$ moves along $\phi_{4}$. This gives $\beta$.

(2) $\sigma$ and $\phi_{5}$ remain fixed while $\phi_{4}$ moves along $\phi_{5}$. This gives $\gamma$.

(3) All remain fixed. This gives $\beta \cap \gamma$.

Lemma 6.11 Each triple point of $\left(\widetilde{X}_{0}\right)_{\text {red }}$ lies in some $Q_{j}$.

PROOF: A triple point is a stable map $f: C \rightarrow \mathcal{F}_{x} i$ where $C$ has at least three double points. The only possibility is $C=\sigma \cup \psi \cup \phi_{4} \cup \phi_{5}$, which corresponds to a point $\beta \cap \gamma$ as in the proof of the previous lemma.

We now quote what seems to be folklore.

Theorem 6.12 $\mathcal{M}_{\text {Ell }}$ is simply connected.

PROOF: This can be found at https://mathoverflow.net/questions/105047/fundamental-group-of-the-moduli-stackof-elliptic-curves

Lemma 6.13 Every double curve of $\widetilde{X}_{0}$ that does not lie in $\sum Q_{j}$ meets at least one $Q_{j}$ and dominates $E$.

PROOF: We can work with the universal curve $\mathcal{E}$. Since $\mathcal{M}_{E l l}$ is simply connected, every geometric component of $\widetilde{X}_{0}$ and every double curve is defined over $\mathcal{M}_{\text {Ell }}$.

Suppose that $A$ is a double curve meeting no $Q_{j}$. Then $A$ parametrizes stable maps $f: \sigma \cup \phi_{4}+\phi_{5} \rightarrow \mathcal{F}_{\xi}$ where $\sigma \cap \phi_{4}$ is never equal to $\sigma \cap \phi_{5}$. This defines a subtraction morphism $t: A \rightarrow E$ by $t(f)=\left(\sigma \cap \phi_{4}\right)-\left(\sigma \cap \phi_{5}\right)$ which is never zero, and so is constant and defines a non-zero point on $E$. But on the universal curve the only rational point is zero.

Lemma 6.14 $\tilde{X}_{0}$ is reduced and $K_{\tilde{X}} \sim \sum Q_{j}$.

PROOF: We argue by induction.

Start by considering a neighbourhood in $\widetilde{X}$ of some $Q_{j}=Q$. It meets two components $L, M$ of $\widetilde{X}_{0}$. Assume, as our induction hypothesis, that $L$ has multiplicity 1 in $\widetilde{X}_{0}$. Since $\widetilde{X}_{0} \sim 0$ as divisors on $\widetilde{X}$, we can write

$$
\widetilde{X}_{0}=L+m M+q Q, K_{\widetilde{X}} \sim p M+r Q
$$


where $m, q, p, r \in \mathbb{Z}$ and $m, q>0$. Say $Q \cap M=\gamma, Q \cap L=\beta$. Note that $L . \beta=\left(\beta_{Q}\right)^{2}=0$ and $M \cdot \gamma=\left(\gamma_{Q}\right)^{2}=0$.

So $\widetilde{X}_{0} \cdot \beta=0$ gives $m=q a$ and $\widetilde{X}_{0} \cdot \gamma=0$ gives $q b=1$, so that $q=b=1$ and $m=a$.

The adjunction formula for the curve $\gamma$ in the 3 -fold $\widetilde{X}$ is $2 g(\gamma)-2=K_{\tilde{X}} \cdot \gamma+$ $\operatorname{deg} \mathcal{N}_{\gamma / \tilde{X}}$, which gives $-2=r .(-1)-1$, so $r=1$ and we have

$$
\widetilde{X}_{0}=L+m M+Q, K_{\widetilde{X}} \sim p M+Q .
$$

The adjunction formula applied to $\beta$ gives $p=2(m-1)$, so that $K_{\widetilde{X}} \sim$ $2(m-1) M+Q$.

Suppose next that $A$ is a double curve that lies in $L$ and meets $Q$. So $A$ is a connected component of $L \cap M$, meeting $Q_{1}, \ldots, Q_{s}$, say. Pick a sufficiently small neighbourhood $U$ of $A$ that contains $Q_{1}, \ldots, Q_{s}$.

The adjunction formula for $L$ gives $K_{L} \sim(m-2) A$ (in the neighbourhood $U \cap L$ of $A$ in $L)$. There are $(-1)$-curves $\beta_{1}, \ldots, \beta_{s}$ in this neighbourhood; they satisfy $\beta_{i} \cdot A=1$ and $\beta_{i} \cdot K_{L}=-1$, and so $m=1$ and then $K_{\widetilde{X}} \sim Q$. Since $\widetilde{X}_{0}$ is connected in codimension one, this completes the proof of Lemma 6.14.

Now drop the distinction between the $D_{i}$ and the $F_{k}$. The adjunction formula shows that $-\left.K_{D_{i}} \sim\left(\sum_{j \neq i} D_{j}\right)\right|_{D_{i}}$.

Lemma 6.15 If $L$ is a smooth projective surface and $B \in|-K+L|$ is smooth, but maybe disconnected, then there are three possibilities.

(1) $B$ is connected, $g(B)=1$ and $L$ is rational.

(2) $B$ is connected, $g(B)=1, L$ is elliptic ruled and $B$ is a bisection of the ruling.

(3) $B$ has just two connected components $B_{1}$ and $B_{2}, g\left(B_{i}\right)=1, L$ is elliptic ruled and each $B_{i}$ is a section of the ruling.

PROOF: This is easy and well known.

Since $X_{0}$ has a $\mathbb{G}_{m}$-action, $D_{0}$ contains at most one curve of genus $\geq 1$. Since $D_{0}$ meets $\widetilde{X}_{0}-D_{0}$, the divisors $D_{0}, \ldots, D_{r}$ form a chain of surfaces, where, for $r \geq i \geq 1$, each $D_{i} \cap D_{i-1}$ is a curve $A_{i}$ of genus 1 . Moreover, $A_{1}$ is an anticanonical divisor on $D_{0}, A_{r}$ is an anticanonical divisor on $D_{r}$ and, for $1 \leq i \leq r-1$, $A_{i}+A_{i+1}$ is an anticanonical divisor on $D_{i}$. So $D_{1}, \ldots, D_{r-1}$ are elliptic ruled and $D_{r}$ is rational or elliptic ruled.

Lemma 6.16 Each $A_{i}$ meets exactly one $Q_{j}$ and is isomorphic to $E$.

PROOF: If $A_{i}$ meets $s$ of the $Q_{j}$ then the subtraction morphism $t: A_{i} \rightarrow E$ has a fibre $t^{-1}(0)$ consisting of $s$ points. So $t$ is étale of degree $s$ in characteristic zero. Then $t$ is étale of degree $s$ universally. Then the kernel of the dual morphism 
$t^{\vee}: E \rightarrow \operatorname{Pic}^{0}\left(A_{i}\right)$ is a finite étale subgroup scheme of $E$ of order $s$. But on the universal curve the only such subgroup scheme is trivial, so $s=1$.

So $\widetilde{X}_{0}=\sum_{0}^{r} D_{i}+\sum_{1}^{r} Q_{i}$ where the $D_{i}$ form a chain of surfaces that intersect in copies $A_{1}, \ldots, A_{r}$ of $E$ and $Q_{j}$ intersects $D_{j-1}$ in the curve $\beta_{j}$ and $D_{j}$ in the curve $\gamma_{j}$. Both $\beta_{j+1}$ and $\gamma_{j}$ are $(-1)$-curves on $D_{j}$. The dual complex of $\widetilde{X}_{0}$ is shown in Figure 2.
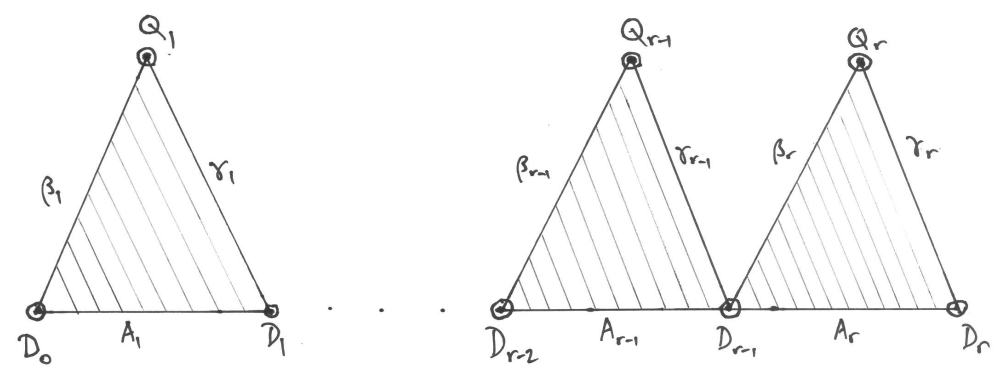

Figure 2: The dual complex of $\widetilde{X}_{0}$

Lemma 6.17 $D_{r}$ is geometrically rational.

PROOF: If not, then $A_{r}$ is a bisection of the ruling on $D_{r}$, so that there is a universally defined morphism $E \rightarrow E$ of degree 2, which is impossible.

Corollary 6.18 $X_{0}$ has a simply elliptic singularity. Say its degree is $d$; then $d \leq 4$. Its multiplicity is $\max \{2, d\}$ and its embedding dimension is $\max \{3, d\}$.

PROOF: We know that $K_{D_{0}} \sim-A_{1}$; it follows at once that $X_{0}$ is simply elliptic. That is, the exceptional locus in its minimal resolution is a single elliptic curve. Since $X_{0}$ has a $\mathbb{G}_{m}$-action it is isomorphic to a line bundle over $A_{1}$, which is isomorphic to $E$.

There is a Cartesian diagram

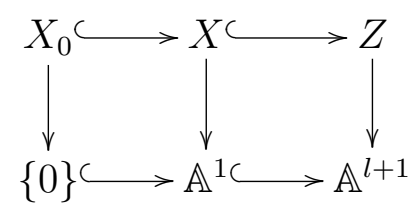

whose horizontal arrows are closed embeddings; it exhibits $X_{0}$ as an LCI singularity. From the well known structure of these homogeneous co-ordinate rings it 
follows that $d \leq 4$ and that the multiplicity and embedding dimension of $X_{0}$ are as described.

We now let $d$ denote the degree of the simply elliptic singularity $X_{0}$. Recall that $l$ is the rank of the group $G$.

Proposition $6.19 d=9-l$.

PROOF: The point is to show that $X_{0}$ has, first, the correct embedding dimension and, then, the correct multiplicity.

Recall that $Z \cong \mathbb{A}^{l+3}$ and that $X \rightarrow \mathbb{A}^{1}$ is obtained from a morphism $\pi: Z \rightarrow$ $[\widehat{Y} / W]$ that preserves $\mathbb{G}_{m}$-orbits by restricting to the line in $[\widehat{Y} / W]$ that goes through the origin of $Y$. So $X_{0}$ is embedded in $Z$ as the fibre over the vertex of $[\widehat{Y} / W]$. By Looijenga's result (Theorem 6.2 above), $[\widehat{Y} / W]$ is isomorphic to $\mathbb{A}^{l+1}$ and the weights of the $\mathbb{G}_{m}$-action on $[\widehat{Y} / W]$ are the coefficients in the biggest root in the affine Dynkin diagram. These are given by Table 1, in which each exponent denotes the multiplicity of the relevant weight.

Table 1: Coefficients in the biggest root in the affine diagram

$$
\begin{array}{lcccccc}
E_{8}: & 1 & 2^{2} & 3^{2} & 4^{2} & 5 & 6 \\
E_{7}: & 1^{2} & 2^{3} & 3^{2} & 4 & & \\
E_{6}: & 1^{3} & 2^{3} & 3 & & & \\
D_{5}: & 1^{4} & 2^{2} & & & &
\end{array}
$$

We also need to know the weights of the $\mathbb{G}_{m}$-action coming from the action of the centre $\zeta\left(\Lambda_{5}\right)$ of $\Lambda_{5}$. Note that $\zeta\left(\Lambda_{5}\right) \cong \mathbb{G}_{m}$.

Lemma 6.20 The weights of the $\zeta\left(\Lambda_{5}\right)$-action on $Z$ are given in Table 2 .

Table 2: Weight multiplicities for the action of $\zeta\left(\Lambda_{5}\right)$ on $Z$

$$
\begin{array}{llllll}
E_{8}: & 1^{2} & 2^{3} & 3^{3} & 4^{2} & 5 \\
E_{7}: & 1^{4} & 2^{4} & 3^{2} & & \\
E_{6}: & 1^{6} & 2^{3} & & & \\
D_{5}: & 1^{8} & & & &
\end{array}
$$

PROOF: As before, we have $\underline{\mathfrak{g}}_{5}=\underline{\mathfrak{l}}_{5} \oplus \underline{\mathfrak{n}}^{+} \oplus \underline{\mathfrak{n}}^{-}$. This represents the fact that the $G / P_{5}$-bundle associated to $\Xi_{5}$ has a section $\sigma$ with $[\sigma]=-\alpha_{5}^{\vee}$ (see Remark 3.13).

Consider the grading of $\underline{\mathfrak{n}}^{+}=\oplus_{0<i \leq r} \underline{\mathfrak{n}}_{i}^{+}$defined by the action of $\mathbb{G}_{m}$ via the cocharacter $\varpi_{5}^{\vee}$. Then the fibre $\mathfrak{n}_{i}^{+}$of $\underline{\mathfrak{n}}_{i}^{+}$is the direct sum $\mathfrak{g}_{\alpha}$ of root spaces where 
$\alpha$ runs over the set $I_{5}(i)$ of positive roots such that $\alpha_{5}$ has coefficient exactly $i$ in $\alpha$, and $r$ is the multiplicity of $\alpha_{5}$ in the biggest root.

Note that $\mathfrak{n}_{i}^{+}$is a representation of $\Lambda_{5}$, so that $\sum_{\alpha \in I_{5}(i)} \alpha$ is trivial on the derived subgroup of $\Lambda_{5}$. Therefore $\sum_{\alpha \in I_{5}(i)} \alpha=n_{i} \varpi_{5}$, where $n_{i}$ depends on $i$ and $G$.

Put $d_{i}=\operatorname{deg} \underline{\mathfrak{n}}_{i}^{+}$, so that $d_{i}=\left(\sum_{\alpha \in I_{5}(i)} \alpha\right) \cdot \alpha_{5}^{\vee}=n_{i}$. But $n_{i}\left(\varpi_{5} \cdot \varpi_{5}^{\vee}\right)=$ $\sum_{\alpha \in I_{5}(i)}\left(\alpha \cdot \varpi_{5}^{\vee}\right)=i \# I_{5}(i)$, and then an inspection of Bourbaki's planches shows that $r$ and the $d_{i}$ are given as follows:

$$
\begin{array}{ccccc}
E_{8}: & r=5, \quad\left(d_{1}, \ldots, d_{5}\right) & = & (2,3,3,2,1) \\
E_{7}: & r=3, \quad\left(d_{1}, d_{2}, d_{3}\right) & = & (4,4,2) \\
E_{6}: & r=2, \quad\left(d_{1}, d_{2}\right) & = & (6,3) \\
D_{5}: & r=1, \quad d_{1} & = & 8 .
\end{array}
$$

From the description above of $\mathfrak{g}$, we know that $H^{1}\left(E, \underline{\mathfrak{n}}^{+}\right)=\oplus_{i} H^{1}\left(E, \underline{\mathfrak{n}}_{i}^{+}\right)=0$, so by Riemann-Roch $\operatorname{dim} H^{0}\left(\bar{E}, \underline{\mathfrak{n}}_{i}^{+}\right)=\chi\left(E, \underline{\mathfrak{n}}_{i}^{+}\right)=d_{i}$, and as $\zeta\left(\Lambda_{5}\right)=\mathbb{G}_{m}$ acts with weight $i$ on the bundle $\underline{\mathfrak{n}}_{i}^{+}$and so on $H^{0}\left(E, \underline{\mathfrak{n}}_{i}^{+}\right)$, the lemma is proved.

Remark: Note that $H^{0}\left(E, \underline{\mathfrak{g}}_{5}\right)=H^{0}\left(E, \underline{\mathfrak{l}}_{5}\right) \oplus H^{0}\left(E, \underline{\mathfrak{n}}^{+}\right)$and $\operatorname{dim} H^{0}\left(E, \underline{\mathfrak{l}}_{5}\right)=1$, since $\lambda_{5}$ is determined by a $G L_{p} \times G L_{q} \times G L_{s}$ bundle $\left(\eta_{p}, \eta_{q}, \eta_{s}\right)$ with $\operatorname{det} \eta_{p}=$ $\operatorname{det} \eta_{q}=\operatorname{det} \eta_{s}$ of degree 1 and $(p, q, s)=(1,5, l-4)$. This provides another proof that $\operatorname{dim} H^{0}\left(E, \underline{\mathfrak{g}}_{5}\right)=l+4$.

Lemma 6.21 If $f: A \rightarrow B$ is an injective $k$-algebra homomorphism of positively graded domains such that the corresponding morphism $F: \operatorname{Spec} B \rightarrow \operatorname{Spec} A$ takes $\mathbb{G}_{m}$-orbits to $\mathbb{G}_{m}$-orbits, then there is an integer $e=e(f)>0$ such that $f\left(A_{n}\right) \subset B_{\text {en }}$ for all $n$. That is, $f$ multiplies degrees by $e$.

PROOF: That $F$ preserves $\mathbb{G}_{m}$-orbits means that for every $n$, there exists $n^{\prime}$ such that $f\left(A_{n}\right) \subset B_{n^{\prime}}$. Suppose that $x \in A_{m}$ and $y \in A_{n}$; then

$$
n \operatorname{deg} f(x)=\operatorname{deg} f\left(x^{n}\right)=\operatorname{deg} f\left(y^{m}\right)=m \operatorname{deg} f(y),
$$

so that $\operatorname{deg} f(x) / \operatorname{deg} f(y)=m / n=\operatorname{deg} x / \operatorname{deg} y$, which proves the lemma.

The next result is crucial. To prove it we must use the fact that has now been proved, that $X_{0}$ is a simply elliptic LCI singularity and so has embedding dimension at most 4 .

Proposition 6.22 For each group $G=E_{8}, \ldots, D_{5}$ the integer $e$ is equal to 1 .

PROOF: We check the cases separately. For $E_{8}$ the tables above show that $A, B$ are weighted polynomial rings, as follows: $A=k\left[1,2^{2}, 3^{2}, 4^{2}, 5,6\right]$ and $B=$ $k\left[1^{2}, 2^{3}, 3^{3}, 4^{2}, 5\right]$. Then the fact that the fibre $X_{0}$ over the origin of $\operatorname{Spec} B \rightarrow$ $\operatorname{Spec} A$ has embedding dimension at most 4 at its unique singular point forces $e=1$ in this case, and $e=1$ in all the other cases for exactly similar reasons. 
Remark: It is possible instead to give a case-free proof of this proposition via the interpretation of the line bundle $\mathcal{L}$ on $\mathcal{Y}$ as a determinant line bundle; we plan to return to this approach [GS].

Therefore $X_{0}$ is embedded $\mathbb{G}_{m}$-equivariantly as a complete intersection in an affine space as follows:

$$
\begin{aligned}
& E_{8}: X_{0}=(6) \quad \hookrightarrow \quad \mathbb{A}^{3}(1,2,3) \\
& E_{7}: X_{0}=(4) \quad \hookrightarrow \quad \mathbb{A}^{3}\left(1^{2}, 2\right) \\
& E_{6}: X_{0}=(3) \quad \hookrightarrow \quad \mathbb{A}^{3}\left(1^{3}\right) \\
& D_{5}: X_{0}=(2,2) \hookrightarrow \hookrightarrow \quad \mathbb{A}^{4}\left(1^{4}\right) .
\end{aligned}
$$

It follows that $X_{0}$ has the correct multiplicity, and the proof of Proposition 6.19 is complete.

Lemma 6.23 The morphisms $\widetilde{X} \rightarrow \mathbb{A}_{k}^{1}$ and $X \rightarrow \mathbb{A}_{k}^{1}$ cover the standard action of $\mathbb{G}_{m}$ on $\mathbb{A}_{k}^{1}$.

PROOF: It is enough to prove the lemma for $X \rightarrow \mathbb{A}_{k}^{1}$. However, this is nothing but Proposition 6.22.

Next, we recover the result whose proof was sketched by Helmke and Slodowy [HS3] on deformations of simply elliptic singularities. In fact the results of Hirokado [Hi], Corollary 4.3 and his calculation of $T^{1}$ (the tangent space to a miniversal deformation space) in the proof of [Hi] Theorem 4.4 permit us to prove this in almost all characteristics.

From now on we make the following assumption: char $k=0$ if $G=D_{5}$ and $\operatorname{char} k \neq 9-l$ if $G=E_{l}$ with $l=6$ or 7 . Note that when $G=E_{8}$ this excludes nothing.

Hirokado shows that then simply elliptic singularities of degree at most 3 are classified exactly as in characteristic zero (in particular, they are quasihomogeneous) and that the structure of their miniversal deformation spaces is uniform across characteristics. In particular a miniversal deformation space $T$ of such a singularity has a $\mathbb{G}_{m}$-action and there is a hypersurface $S$ in $T$ where the $\mathbb{G}_{m}$-action has positive weights. The complementary line is where the singularity remains simply elliptic but the elliptic curve varies.

Theorem 6.24 The morphism $Z \rightarrow[\widehat{Y} / W]$ is the positive weight part of a $\mathbb{G}_{m}$-equivariant miniversal deformation of $X_{0}$.

PROOF: Suppose that $V \rightarrow S$ is the positive weight part of a $\mathbb{G}_{m}$-equivariant miniversal deformation of $X_{0}$. Certainly $Z \rightarrow[\widehat{Y} / W]$ is $\mathbb{G}_{m}$-equivariant and of positive weight, so there is a $\mathbb{G}_{m}$-equivariant morphism $a:[\widehat{Y} / W] \rightarrow S$ and a Cartesian square

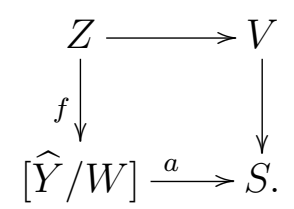


The derivative of the morphism $a:[\widehat{Y} / W] \rightarrow S$ at the vertex of $[\widehat{Y} / W]$ is determined uniquely, and then $a$ is determined uniquely by its $\mathbb{G}_{m}$-equivariance. Inspection shows that the weights of the $\mathbb{G}_{m}$ action on the affine spaces $[\widehat{Y} / W]$ and $S$ are the same, so that $a$ is an isomorphism.

In particular, the family $X \rightarrow \mathbb{A}^{1}$ is induced by a unique $\mathbb{G}_{m}$-equivariant morphism $\phi: \mathbb{A}^{1} \rightarrow S$. Recall that the base $\mathbb{A}^{1}$ of $X \rightarrow \mathbb{A}^{1}$ is the generator in the cone $[\widehat{Y} / W]$ that passes through the origin $0_{Y}$ of $Y$.

Proposition 6.25 (1) Under the morphism $a$, the line $\mathbb{A}^{1}=\operatorname{Spec} k[t]$ in $[\widehat{Y} / W]$ is mapped to a line $\ell=\operatorname{Spec} k[x]$ in $S$ where for $x \neq 0$ the surface $V_{x}$ has a $d u$ Val singularity of type $G$.

(2) For $t \neq 0, \widetilde{X}_{t}$ is the minimal resolution of $X_{t}$; it contains a $(-2)$ configuration $\Gamma_{t}$ of type $G$.

PROOF: Suppose first that $k=\mathbb{C}$. Then, by Theorem (7.7) of [L2], the monodromy around $V_{s}$ is the full Weyl group. That is, take a small neighbourhood $U$ of $s$ in $S$ and consider the complement $U-\Delta$ of the discriminant; then $\pi_{1}(U-\Delta)$ acts on the cohomology of the generic fibre as the full Weyl group. Since $V_{s}$ has at most du Val singularities, (1) is proved in this case. Specialization then proves it for all fields.

(2) is a corollary of (1) and Theorem 3.11.

Remark: It would be desirable to have a conceptual proof of this proposition, maybe as follows. The copy of $\mathbb{A}^{1}=\operatorname{Spec} k[t]$ that appears is the line through the origin $0 \mathcal{Y}$ of $\mathcal{Y}$ so that the bundles parametrized by $\left.\mathcal{Z}\right|_{t \neq 0}$ are unipotent (and semi-stable).

On the other hand, general considerations of deformation theory show that the automorphism group of each of these bundles has dimension strictly less than that of $\Xi_{5}$, which is $l+4$. Therefore they are subregular or regular; if we could prove that subregular bundles do arise over points of $\mathbb{A}^{1}-\{0\}$ (that is, if we could prove directly that $\Xi_{5}$ deforms to a subregular unipotent semi-stable bundle) then Theorem 3.11 could be applied, assuming that $E$ is uniformizable; the result would follow for all $E$ by specialization. As it is, we only know how to deduce the existence of such a subregular deformation from Proposition 6.25.

\section{Proposition $6.26 r=1$.}

PROOF: Since $K_{\widetilde{X}} \sim \sum Q_{i}$, the divisors $D_{1}, \ldots, D_{r}$ appear with discrepancy zero in the resolution $\widetilde{X}$ of the 3 -fold $X$. They also dominate the closed point 0 that represents $\xi$. So it is enough to exhibit a partial resolution $X^{\prime} \rightarrow X$ which has $\mathrm{c}-\mathrm{dV}$ singularities and in which there is only one divisor of discrepancy zero that dominates the origin.

For this, note that from Lemma 6.18 and the proof of Proposition 6.17 it 
follows that the Cartesian diagram

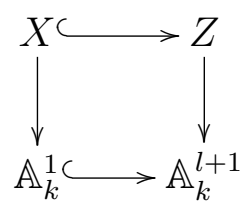

exhibits $X$ as a homogeneous complete intersection of codimension $l$ in some affine space.

For the groups $E_{8}, E_{7}, E_{6}$ and $D_{5}, X$ is, respectively, a homogeneous sextic in $\operatorname{Spec} k\left[1^{2}, 2,3\right]=\mathbb{A}_{k}^{4}$, a quartic in Spec $k\left[1^{2}, 2\right]$, a cubic in Spec $k\left[1^{4}\right]$ and a complete intersection of two quadrics in Spec $k\left[1^{5}\right]$. We know that for $t \neq 0$ the fibre $X_{t}$ has du Val singularities, so that in each case the subvariety Proj $\mathcal{O}_{X}$ in the appropriate weighted projective space has only du Val singularities and is a del Pezzo surface of degree $9-l$. Therefore the weighted blow-up $X^{\prime}=$ $\operatorname{Proj} \mathcal{O}_{X}[t] \rightarrow X$ centred at 0 has a unique exceptional divisor, namely $\operatorname{Proj} \mathcal{O}_{X}$. Since this divisor is Cartier and has du Val singularities, it follows that $X^{\prime}$ has only c-dV singularities and is is a partial resolution of the kind that we sought.

So $\widetilde{X}_{0}=D_{0}+D_{1}+Q$ where $D_{1}$ is rational and $A=D_{0} \cap D_{1}$ is an effective anticanonical divisor on each of $D_{0}, D_{1}$. Say $\beta=Q \cap D_{0}, \gamma=Q \cap D_{1}$. Then each of $\beta, \gamma$ is a $(-1)$-curve.

We define a $(-1,-2)$-chain of length $s$ on $D_{0}$ to be an $s$-tuple $\left(\delta_{1}, \ldots, \delta_{s}\right)$ of smooth rational curves on $D_{0}$ where $\delta_{1}^{2}=-1, \delta_{i}^{2}=-2$ for $i \geq 2, \delta_{1} \cdot A=1$, $\delta_{i} . A=0$ for $i \geq 2$ and the configuration $A, \delta_{1}, \ldots, \delta_{s}$ is a chain.

Lemma 6.27 The only complete curves on $D_{0}$ are $A$ and those curves that occur in a $(-1,-2)$-chains. Exactly one of these chains contains $\beta$.

PROOF: This follows from the adjunction formula, the fact that $A \sim-K_{D_{0}}$ and the fact that any configuration of complete curves on $D_{0}$ is negative definite.

It follows that there is a birational contraction $\widetilde{X} \rightarrow \widetilde{X}^{+}$where $Q$ is contracted onto the curve $\gamma$ in $D_{1}$ and where the images in $\widetilde{X}^{+}$of the curves in the $(-1,-2)$ chains in $D_{0}$ can be flopped successively onto $D_{1}$, say via $\widetilde{X}^{+}-\rightarrow \widetilde{X}^{-}$, to give $\widetilde{X}^{-} \rightarrow \mathbb{A}^{1}$ where $\widetilde{X}_{0}^{-}=D_{0}^{-}+D_{1}^{-}$is semi-stable, $K_{\widetilde{X}^{-}} \sim 0, D_{0}^{-}$is the strict transform of $D_{0}$ and is the minimal resolution of $X_{0}, D_{1}^{-}$is the strict transform of $D_{1}$ and is a weak del Pezzo surface on which $A$ is an anti-canonical curve and the flopped curves on $D_{1}^{-}$form a configuration of $(-1,-2)$-chains which is isomorphic to the configuration of $(-1,-2)$-chains on $D_{0}$.

Moreover, these birational birational transformations are $\mathbb{G}_{m}$-equivariant, so that $\widetilde{X}^{-}$has a $\mathbb{G}_{m}$-action that covers the standard action on $\mathbb{A}_{k}^{1}$ and is free on $D_{0}^{-}-A^{-}$, where $A^{-}$is the strict transform of $A$. The action covers the standard action on $\mathbb{A}_{k}^{1}$ and so is free on $\widetilde{X}^{-}-D_{1}^{-}$. 
Since $\left(A_{D_{1}^{-}}^{-}\right)^{2}=-\left(A_{D_{0}^{-}}^{-}\right)^{2}$, it follows that $D_{1}^{-}$is a weak del Pezzo surface of degree $d=9-l$.

It is easy to see that if $\mathbb{G}_{m}$ acts on a del Pezzo surface $S$, weak or not, and preserves a smooth member of $\left|-K_{S}\right|$, then the action on $S$ is trivial. So $\mathbb{G}_{m}$ acts freely on $\widetilde{X}^{-}-D_{1}^{-}$and trivially on the Cartier divisor $D_{1}^{-}$.

The next lemma and its proof are taken almost without change from a paper by Bass and Haboush [BH].

Lemma 6.28 Assume that $S$ is a normal $k$-variety and that $D$ is a complete relatively LCI closed subscheme of $S$ that contains every complete subscheme of $S$. Assume also that there is a $\mathbb{G}_{m}$-action on $S$ that is trivial on $D$ and free on $S-D$ and that every closed $\mathbb{G}_{m}$-invariant subscheme of $S$ meets $D$. Then there is a $\mathbb{G}_{m}$-equivariant isomorphism $S \rightarrow N_{D / S}$ that identifies $D$ with the zero section of $N_{D / S}$.

PROOF: The aim is to reduce this to a situation where the arguments of $[\mathrm{BH}]$ can be applied.

We need to show that there is a $\mathbb{G}_{m}$-linearized locally free sheaf $\mathcal{A}$ on $D$ such that $S \cong$ Spec Sym$^{*} \mathcal{A}$.

Choose an open cover $S=\cup_{i \in I} S_{i}$ by affine $\mathbb{G}_{m}$-invariant open subschemes $S^{(i)}=\operatorname{Spec} R^{(i)}$ such that each $D^{(i)}=D \cap S^{(i)}$ is, if non-empty, defined by a regular sequence of length $r=\operatorname{codim}(D, S)$ in $R^{(i)}$. Say $J=\left\{j \in I \mid D^{(j)} \neq \emptyset\right\}$. Then $S-\cup_{j \in J} S^{(j)}$ is a closed $\mathbb{G}_{m}$-invariant subscheme of $S$ that is disjoint from $D$, so is empty. So we can suppose that each $D^{(i)}$ is non-empty.

The existence of the $\mathbb{G}_{m^{-}}$-action is equivalent to a $\mathbb{Z}$-grading $R^{(i)}=\oplus_{n \in \mathbb{Z}} R_{n}^{(i)}$. Since every closed $\mathbb{G}_{m}$-invariant subscheme of $S$ meets $D$, the modules $R_{n}^{(i)}$ vanish for $n<0$. and the ideal of $D^{(i)}$ in $S^{(i)}$ is $R_{+}^{(i)}=\oplus_{n>0} R_{n}^{(i)}$. So $D^{(i)}=\operatorname{Spec} R_{0}^{(i)}$, where we regard $R_{0}^{(i)}$ both as a subring of $R^{(i)}$ and as the quotient $R^{(i)} / R_{+}^{(i)}$.

That is, there is a unique $\mathbb{G}_{m}$-equivariant retraction $r_{i}: S^{(i)} \rightarrow D^{(i)}$. Since they are unique, these retractions glue to a $\mathbb{G}_{m}$-equivariant retraction $r: S \rightarrow D$. Note that, as a subscheme of $S, D$ contains all the closed $\mathbb{G}_{m}$-orbits in $S$.

Now we follow $[\mathrm{BH}]$, pp. 474 et seq. We have $S=\operatorname{Spec} \mathcal{R}, \mathcal{R}=\oplus_{n \geq 0} \mathcal{R}_{n}$, a graded sheaf of $\mathcal{O}_{D}$-algebras. Set $\mathcal{I}=\oplus_{n>0} \mathcal{R}_{n}$, the ideal sheaf of the subscheme $D$ of $S, \mathcal{N}^{\vee}=\mathcal{I} / \mathcal{I}^{2}$ and $Y=$ Spec Sym* $\mathcal{N}^{\vee}$, the normal bundle. The tautological $\mathbb{G}_{m}$-equivariant morphism $u: S \rightarrow Y$ fits into a $\mathbb{G}_{m, D}$-equivariant commutative diagram

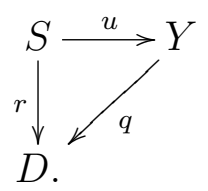

Then the argument of $[\mathrm{BH}]$, p. 474, applies directly to show that $u$ is étale.

Let $D_{0} \hookrightarrow Y$ be the zero section of $q$.

We now claim that $u$ is finite. To see this, take $\widetilde{S}$ to be the normalization of $Y$ in the function field of $S$. Then there is a $\mathbb{G}_{m}$-equivariant open embedding 
$S \hookrightarrow \widetilde{S}$ and a finite dominant morphism $\nu: \widetilde{S} \rightarrow Y$ extending $u$. Say $V=\widetilde{S}-S$; this is closed and $\mathbb{G}_{m}$-invariant in $Y$. Since $D \subset S$ and $D$ is complete, $D$ is disjoint from $V$. Assume that $V \neq \emptyset$; then, over any affine chart of $Y$, there is a $\mathbb{G}_{m}$-invariant function $f$ on $\widetilde{S}$ such that $\left.f\right|_{D}=0$ and $\left.f\right|_{V}=1$. However, $\mathcal{O}_{\widetilde{S}}^{\mathbb{G} m}=\mathcal{O}_{D}$, so that $V=\emptyset$ and the claim is established.

Then $u^{-1}\left(D_{0}\right)=D \coprod D^{\prime}$ with $D^{\prime}$ finite and étale over $D_{0}$. The argument just given, to show that $V=\emptyset$, can now be applied to show that $D^{\prime}=\emptyset$. So $u$ has degree 1 over $D$, and so is of degree 1 everywhere. This proves Lemma 6.28.

Corollary 6.29 (1) There are $\mathbb{G}_{m}$-equivariant isomorphisms $\widetilde{X}^{-} \rightarrow N_{D_{1}^{-} / \widetilde{X}^{-}}$ and $N_{D_{1}^{-} / \tilde{X}^{-}} \rightarrow \omega_{D_{1}^{-}}$.

(2) $X$ is the cone over the anti-canonical model of $D_{1}^{-}$.

PROOF: The first isomorphism of (1) is a special case of the lemma and the second is a consequence of the adjunction formula. (2) is an immediate consequence.

Proposition 6.30 Suppose that $D$ is a weak del Pezzo surface of degree $9-l$ and contains a $(-2)$-configuration $\Delta$ of type $G$. Then

(1) $D$ contains a unique line $L$;

(2) if $\delta_{1}, \ldots, \delta_{l}$ are the irreducible components of $\Delta$, numbered as in Bourbaki's planches, then $L$ meets $\Delta$ in $\delta_{l}$ and in no other component;

(3) $\left\{\delta_{1}, \ldots, \delta_{l}, L\right\}$ is a $\mathbb{Z}$-basis of $\mathrm{NS}(D)$;

(4) given a smooth member $A$ of $\left|-K_{D}\right|, D$ is obtained by embedding $A$ in $\mathbb{P}^{2}$ via $\left|3\left[0_{A}\right]\right|$ and then making $l$ successive blow-ups, with the centre of each blow-up being the origin $0_{A}$ on the strict transform of $A$.

PROOF: This is well known, but we include a proof for lack of a convenient reference.

Existence of a line: $D$ is a specialization of a smooth del Pezzo $D_{t}$ where $-K_{D_{t}}$ is ample, which certainly contains lines. So $D$ does also.

Uniqueness of the line: since $\operatorname{rank} \mathrm{NS}(D)=\operatorname{rank} \Delta+1, \mathrm{NS}(D)_{\mathbb{Q}}$ is spanned by $L$ and $\Delta$ for any line $L$. Moreover, $L . \delta \geq 1$ for any positive root $\delta$ with $\operatorname{Supp} \delta=\Delta$. Put $H=-K_{D}$ and consider cases separately.

$d=1$. Then every simple root has multiplicity $\geq 2$ in the biggest root $\delta_{\text {max }}$, so $L . \delta_{\max } \geq 2$. Then $H^{2}=1=H .\left(L+\delta_{\max }\right)$ and $\left(L+\delta_{\max }\right)^{2} \geq 1$, so that, by the index theorem, $L+\delta_{\max } \sim H \sim M+\delta_{\max }$ for any lines $L, M$. So $L \sim M$ and then $L=M$.

$d \geq 2$. Then $(L+\delta)^{2} \leq 0$ for any line $L$ and any effective root $\delta$, by the index theorem, since $H .\left(L_{\delta}\right)=1$ and $H^{2} \geq 2$. Suppose that $\delta, \epsilon$ are effective roots with $\operatorname{Supp} \delta=\operatorname{Supp} \epsilon=\Delta$ and that $L, M$ are distinct lines; then $L . \delta, M . \delta \geq 1$ and $(L+M+\delta)^{2} \geq 2$. But $H$. $(L+M+\delta)=2 \leq H^{2}$, so that (index) $L+M+\delta \sim$ $H \sim L+M+\epsilon$ and $\delta \sim \epsilon$, which is absurd, and uniqueness is established. 
Moreover, the line $L$ meets $\Delta$ in a simple root $\delta$ of minimal multiplicity (2 if $l=8,1$ otherwise). Then successive contraction of $L, \delta_{l}, \ldots, \delta_{4}, \delta_{3}, \delta_{1}$, in that order, is the inverse of the blow-up described in (4), and also proves (3).

Now we can complete the proof of Theorem 6.7.

At this point we know, by Proposition 6.19 and Corollary 6.72 , that $D_{1}^{-}$is a weak del Pezzo surface of degree $d=9-l$ and that it contains a $(-2)$-configuration $\Delta=\left\{\delta_{1}, \ldots, \delta_{l}\right\}$ of type $G=E_{l}$. So the configuration on $D_{1}^{-}$formed by the strict transform $\gamma^{-}$of $\gamma$ and the flopped curves (see the paragraph following Lemma 6.75 ) form part of the configuration $L, \delta_{1}, \ldots, \delta_{l}$ given by Proposition 6.30 and so form a single $(-1,-2)$-chain. Therefore, by Lemma 6.27 , the flopping curves on $D_{0}$ form a unique $(-1,-2)$-chain on $D_{0}$.

This completes the proof of Theorem 6.7.

\section{Universal families of del Pezzo surfaces}

Theorem 6.7 refers to the line in the line bundle $\mathcal{L} \rightarrow \mathcal{Y}$ that is the fibre over the origin $0_{Y}$, which is in turn defined over an algebraically closed field. However, this extends over the whole of $\mathcal{L}$ as follows.

Recall that $\mathcal{Z}$ is obtained from a chart of $\mathcal{G}_{\mathcal{E}}$ by turning off $\operatorname{Pic}^{1}(\mathcal{E})$ and that $\widetilde{\mathcal{Z}}=\mathcal{Z} \times \mathcal{G}_{\mathcal{E}} \widetilde{\mathcal{G}}_{\mathcal{E}}$. There is a morphism $\rho: \widetilde{\mathcal{Z}} \rightarrow \mathcal{L}$ such that the composite $\widetilde{\mathcal{Z}} \rightarrow \mathcal{Y}$ is smooth and $\rho^{-1}\left(0_{\mathcal{L}}\right)$ coincides with the inverse image of $\Delta \times 0_{\mathcal{Y}}$, where $\Delta$ is the discriminant divisor in $\mathcal{C}_{\text {pre }}$, under the smooth morphism $\widetilde{\mathcal{Z}} \rightarrow \mathcal{C}_{\text {pre }} \times_{\mathcal{M}_{\text {Ell }}} \mathcal{Y}$. So $\rho^{-1}\left(0_{\mathcal{L}}\right)=\mathcal{Q}+\mathcal{D}_{0}+\mathcal{D}_{1}$.

Observe that there is a blowing-down morphism $\widetilde{\mathcal{Z}} \rightarrow \widetilde{\mathcal{Z}}^{+}$where $\mathcal{Q}$ is contracted onto a curve in $\mathcal{D}_{1}$, because $\mathcal{Q}$ is a family of quadrics with normal bundle $\mathcal{O}(-1,-1)$.

Recall the stack $\widetilde{\mathcal{G}}^{+}$from Section 5 .

Lemma $7.1 \quad \widetilde{\mathcal{Z}}^{+}$is isomorphic to $\mathcal{Z} \times{ }_{\mathcal{G}} \widetilde{\mathcal{G}}^{+}$.

PROOF: $\quad \widetilde{\mathcal{Z}} \rightarrow \widetilde{\mathcal{Z}}^{+}$is the contraction of a $\mathbb{P}^{1} \times \mathbb{P}^{1}$-bundle $\mathcal{Q}$ over $\mathcal{Y}$ to a $\mathbb{P}^{1}$-bundle $\gamma$. Fibre by fibre, this is the projection $q: \mathbb{P}^{1} \times \mathbb{P}^{1} \rightarrow \mathbb{P}^{1}$ given by taking the family of $(1,1)$-curves in $S L_{3} / B$ that pass through a fixed point and projecting to the family of lines in $\mathbb{P}^{2}$ through a fixed point. Generically, these $(1,1)$-curves arise as the rational tail $\phi$ in a pre-stable curve $C=E \cup \phi$. From the construction of $\widetilde{\mathcal{G}}^{+}$and the morphism $\psi: \widetilde{\mathcal{G}} \rightarrow \widetilde{\mathcal{G}}^{+}$, this projection is exactly achieved by $\psi$ over $\mathcal{Z}$.

Proposition 7.2 (1) There are sequences of flops $\widetilde{\mathcal{Z}}^{+}-\rightarrow \widetilde{\mathcal{Z}}^{-}$that extend the sequence of flops $\widetilde{X}^{+}-\rightarrow \widetilde{X}^{-}$. 
(2) They fit into a commutative diagram

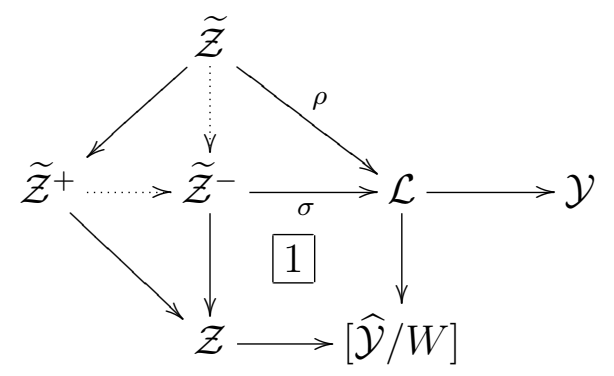

where the square marked 1 is birationally Cartesian.

(3) $\widetilde{\mathcal{Z}}, \widetilde{\mathcal{Z}}^{+}$and $\widetilde{\mathcal{Z}}^{-}$are semi-stable over $\mathcal{L}$. Their restrictions to $\mathcal{L}-0_{\mathcal{L}}$ are isomorphic and smooth over $\mathcal{L}-0_{\mathcal{L}}$.

(4) $\left.\widetilde{\mathcal{Z}}\right|_{0_{\mathcal{L}}}=\mathcal{D}_{0}+\mathcal{D}_{1}+\mathcal{Q},\left.\widetilde{\mathcal{Z}}^{+}\right|_{0_{\mathcal{L}}}=\mathcal{D}_{0}^{+}+\mathcal{D}_{1}^{+},\left.\widetilde{\mathcal{Z}}^{-}\right|_{0_{\mathcal{L}}}=\mathcal{D}_{0}^{-}+\mathcal{D}_{1}^{-}$, and $\mathcal{D}_{i}, \mathcal{D}_{i}^{ \pm}$ and $\mathcal{Q}$ are smooth over $\mathcal{Y}=0_{\mathcal{L}}$.

(5) Each of $\mathcal{D}_{0} \cap \mathcal{D}_{1}$ and $\mathcal{D}_{0}^{ \pm} \cap \mathcal{D}_{1}^{ \pm}$is isomorphic over $\mathcal{Y}$ to $\mathcal{E} \times_{\mathcal{M}_{E l l}} \mathcal{Y}$.

(6) Each fibre of $\mathcal{D}_{0}^{-} \rightarrow \mathcal{Y}$ is the minimal resolution of the minimally elliptic singularity of degree $d$ that belongs to the corresponding elliptic curve.

PROOF: To prove the existence of the flops there is a local problem and a global problem to be solved.

The local problem is to show that under deformation of the ambient 3-fold the curves to be flopped do not disappear and that under specialization they do not break up.

For each successive flop, the irreducible curve $\delta$ to be flopped has normal bundle $\mathcal{N}_{\delta}=\mathcal{O}(-1)^{\oplus 2}$. So $H^{1}\left(\delta, \mathcal{N}_{\delta}\right)=0$ and then $\delta$ survives under deformation.

After $\delta$ has been flopped, the number of irreducible curves to be considered has diminished, and then induction shows that all the curves to be flopped survive under deformation.

A similar argument shows that they do not break up under specialization.

The global problem is to show that there is no monodromy acting on the lattice generated by the classes $\beta, \epsilon_{1}, \ldots, \epsilon_{t}$ in the Chow group of the generic fibre of the family $\mathcal{D}_{0} \rightarrow \mathcal{Y}$. This will follow from the next result.

Proposition $7.3 \mathcal{Y}$ is simply connected.

PROOF: We check first that $\mathcal{E}$ is simply connected.

Suppose that $\pi: \widetilde{\mathcal{E}} \rightarrow \mathcal{E}$ is finite and étale and that $\widetilde{\mathcal{E}}$ is connected.

Since $\mathcal{M}_{E l l}$ is simply connected, by Theorem 6.12 , the origin $0_{\mathcal{E}}$ lifts to $\widetilde{\mathcal{E}}$ and 
we have a 2-commutative diagram

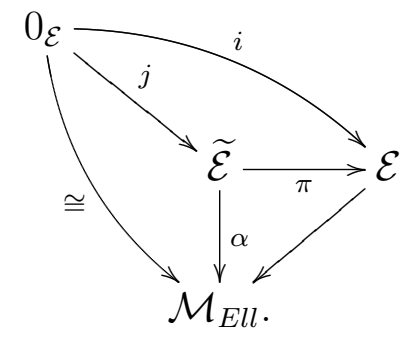

Then $\beta: \widetilde{\mathcal{E}} \rightarrow \mathcal{M}_{E l l}$ is an elliptic curve with identity $0_{\widetilde{\mathcal{E}}}=j\left(0_{\mathcal{E}}\right)$. This is induced by a morphism $\gamma: \mathcal{M}_{E l l} \rightarrow \mathcal{M}_{\text {ell }}$ and we get a commutative diagram

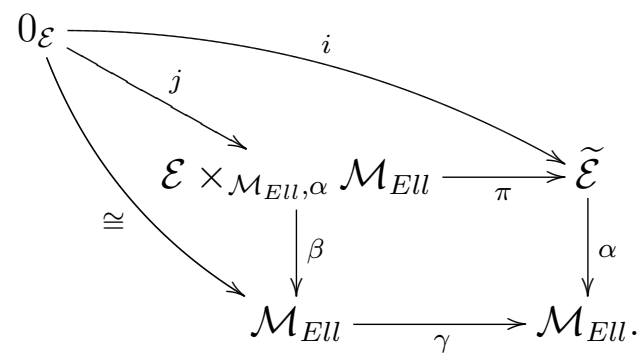

Then $\gamma$ is an isomorphism, and so $\pi$ is an isomorphism and $\mathcal{E}$ is simply connected.

Since $\mathcal{Y}$ is a fibre product of copies of $\mathcal{E}$ it is enough to check that, if $\mathcal{U} \rightarrow \mathcal{M}$ and $\mathcal{V} \rightarrow \mathcal{M}$ have sections $0_{\mathcal{U}}$ and $0_{\mathcal{V}}$ and if $\mathcal{M}, \mathcal{U}$ and $\mathcal{V}$ are simply connected, then so is $\mathcal{U} \times \mathcal{M} \mathcal{V}=\mathcal{W}$, say.

Suppose that $\widetilde{\mathcal{W}} \rightarrow \mathcal{W}$ is finite and étale. The sections $0_{\mathcal{U}}$ and $0_{\mathcal{V}}$ give sections of $\mathcal{W} \rightarrow \mathcal{U}$ and of $\mathcal{W} \rightarrow \mathcal{V}$, which then lift to sections $\sigma: \mathcal{U} \rightarrow \widetilde{\mathcal{W}}$ and $\tau: \mathcal{V} \rightarrow \widetilde{\mathcal{W}}$. This gives a section $(\sigma, \tau): \mathcal{U} \times_{\mathcal{M}} \mathcal{V} \rightarrow \widetilde{\mathcal{W}}$, as required.

This completes the proof of Proposition 7.2.

Corollary 6.29 generalizes straightforwardly to this situation.

Proposition 7.4 (1) Locally on $\mathcal{M}_{\text {Ell }}$ there are $\mathcal{Y}$-isomorphisms $\widetilde{\mathcal{Z}}^{-} \cong \stackrel{\cong}{\rightarrow} N_{\mathcal{D}_{1}^{-} / \widetilde{\mathcal{Z}}^{-}}$ and $N_{\mathcal{D}_{1}^{-} / \widetilde{\mathcal{Z}}^{-}} \cong \omega_{\mathcal{D}_{1}^{-} / \mathcal{Y}}$.

(2) Locally on $\mathcal{M}_{\text {Ell }}$ there is an isomorphism, relative to $\mathcal{Y}$, from $\mathcal{Z}$ to a family of 3-fold cones, namely, to the cone over the anticanonical model of the family $\mathcal{D}_{1}^{-} \rightarrow \mathcal{Y}$ of weak del Pezzo surfaces.

Most of this section is devoted to proving that $\mathcal{D}_{1}^{-} \rightarrow \mathcal{Y}$ is close to being universal.

Let $\tau: \widetilde{\mathcal{Z}}^{-} \rightarrow \mathcal{D}_{1}^{-}$be the projection of $N_{\mathcal{D}_{1}^{-} / \widetilde{\mathcal{Z}}^{-}}$to its base. By construction, there is a smooth morphism $\widetilde{\mathcal{Z}} \rightarrow \widetilde{\mathcal{G}}$; this restricts to give a morphism

$$
\widetilde{\mathcal{Z}}^{0}:=\widetilde{\mathcal{Z}}-\rho^{-1}\left(0_{\mathcal{L}}\right)=\widetilde{\mathcal{Z}}^{-}-\sigma^{-1}\left(0_{\mathcal{L}}\right) \rightarrow \widetilde{\mathcal{G}}^{s s} .
$$

We identify $\mathcal{D}_{1}^{-}$with the zero section of $N_{\mathcal{D}_{1}^{-} / \widetilde{\mathcal{Z}}^{-}}$and $\mathcal{D}_{0}^{-}$with $\tau^{-1}(\mathcal{A})$. 
Proposition 7.5 There is an embedding $\operatorname{Pic}_{F}^{G} \hookrightarrow \operatorname{Pic}_{\widetilde{\mathcal{G}}_{\mathcal{E}}^{s s}}$.

PROOF: A point $P$ of $\widetilde{\mathcal{G}}_{\mathcal{E}}^{s s}$ consists of a $G$-bundle $\Xi$ over an elliptic curve $E$ and a section $\sigma$ of $\mathcal{F}_{\Xi} \rightarrow E$ whose cocharacter $[\sigma]$ vanishes. On the other hand, an element $\varpi$ of $\operatorname{Pic}_{F}^{G}$ gives a line bundle $\mathcal{L}_{\varpi}$ on $\mathcal{F}_{\Xi}$; evaluating $\mathcal{L}_{\varpi}$ at the origin $0_{\sigma}$ of $\sigma$ gives a line, so a line bundle $\mathcal{M}_{\varpi}$ on $\widetilde{\mathcal{G}}_{\mathcal{E}}^{s s}$ with $\mathcal{M}_{\varpi}(P)=\mathcal{L}_{\varpi}\left(0_{\sigma}\right)$. This gives the embedding that was asserted.

Pull back to $\operatorname{Pic}\left(\widetilde{\mathcal{Z}}^{-}-\sigma^{-1}\left(0_{\mathcal{L}}\right)\right)$ via the morphism $\widetilde{\mathcal{Z}}^{-}-\sigma^{-1}\left(0_{\mathcal{L}}\right) \rightarrow \widetilde{\mathcal{G}}^{s s}$; we get a homomorphism $\lambda: \operatorname{Pic}_{F}^{G} \rightarrow \operatorname{Pic}\left(\widetilde{\mathcal{Z}}^{-}-\sigma^{-1}\left(0_{\mathcal{L}}\right)\right)$. Now fix an elliptic curve $E$ over a field $k$ and return to the restriction $\widetilde{X}^{-} \rightarrow \mathbb{A}_{k}^{1}$ of $\widetilde{\mathcal{Z}}^{-}$to the line $\mathbb{A}_{k}^{1}$ in $L$ over the origin $0_{Y}$. For $t \neq 0$, the fibre $\widetilde{X}_{t}^{-}$contains a $(-2)$-configuration $\Delta_{t}$ of type $G=E_{l}$; since $\widetilde{Z}^{-} \cong N_{D_{1}^{-} / \widetilde{Z}^{-}}, \Delta_{t}$ specializes to an isomorphic such configuration $\Delta_{0}$ on $D_{1}^{-}$.

Regard $\widetilde{X}_{t}^{-}$as a surface contained in $\widetilde{\mathcal{Z}}^{-}-\sigma^{-1}\left(0_{\mathcal{L}}\right)$. By Theorem 3.8, $\Delta_{t}$ is identified with a subregular unipotent Springer fibre associated to $G$ and, for any $\varpi \in \operatorname{Pic}_{F}^{G}, \lambda(\varpi)$ is identified with $\varpi$.

We are led to the following result.

Proposition 7.6 There are subgroups $\widetilde{H}$ of $\mathrm{Pic}_{\widetilde{\mathcal{Z}}}$ and $H^{-}$of $\mathrm{Pic}_{\widetilde{\mathcal{Z}}^{-}}$and a commutative diagram with exact rows

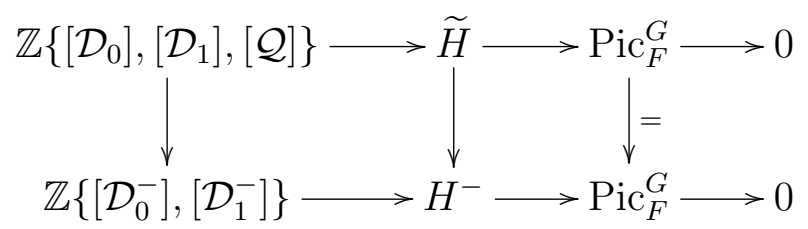

where the two leftmost vertical arrows are induced by the blowing-down $\widetilde{\mathcal{Z}} \rightarrow \widetilde{\mathcal{Z}}^{+}$ and the flop $\widetilde{\mathcal{Z}}^{+}-\rightarrow \widetilde{\mathcal{Z}}^{-}$. In particular, $[\mathcal{Q}] \mapsto 0$.

PROOF: The only remaining point is to check that the obvious homomorphisms $\widetilde{H} \rightarrow \operatorname{Pic}_{\widetilde{\mathcal{Z}}}$ and $H^{-} \rightarrow \mathrm{Pic}_{\widetilde{\mathcal{Z}}^{-}}$are injective. However, this follows from restricting to one of the surfaces $\widetilde{Z}_{t}$, as just described.

Restrict from $\widetilde{\mathcal{Z}}^{-}$to $\mathcal{D}_{1}^{-}$. We get a subgroup $H \subset \mathrm{Pic}_{\mathcal{D}_{1}^{-}}$and, since $\mathcal{A}=$ $\left.\mathcal{D}_{0}^{-}\right|_{\mathcal{D}_{1}^{-}} \sim \omega_{\mathcal{D}_{1}^{-} / \mathcal{Y}}^{-1}$, an exact sequence

$$
\mathbb{Z}[\mathcal{A}] \oplus \mathbb{Z}[\gamma] \longrightarrow H \stackrel{\chi}{\longrightarrow} \operatorname{Pic}_{F}^{G} \longrightarrow 0
$$

such that, for any $\Lambda \in H$ and component $\delta_{i}$ of $\Delta,\left(\Lambda . \delta_{i}\right)_{\mathcal{D}_{1}^{-}}=\left(\chi(\Lambda) \cdot \alpha_{i}^{\vee}\right)$, where $\alpha_{i}^{\vee}$ is the simple coroot corresponding to $\delta_{i}$.

Corollary 7.7 The natural homomorphism $H \rightarrow \operatorname{NS}\left(\mathcal{D}_{1}^{-} / \mathcal{Y}\right)$ of sheaves of commutative groups on $\mathcal{Y}$ is surjective, and $\operatorname{NS}\left(\mathcal{D}_{1}^{-} / \mathcal{Y}\right)$ is constant.

PROOF: It is enough to prove surjectivity for one (geometric) weak del Pezzo surface $D_{1}^{-}$. Choose $D_{1}^{-}$lying over $0_{Y}$; this surface contains a configuration $\Delta_{0}$ 
of type $G$ and a line $\gamma$, and the result now follows from Proposition 6.30 and the fact that $\mathcal{Y}$ is simply connected.

Take $D_{1}^{-}$as in the proof just given. Let $I_{1, l}$ denote the $\mathbb{Z}$-lattice with $\mathbb{Z}$-basis $\left\{\delta_{1}, \ldots, \delta_{l}, \gamma\right\}$ and inner product given by the intersection numbers on $D_{1}^{-}$; then $I_{1, l}$ is isomorphic to the standard odd unimodular hyperbolic lattice of rank $1+l$ and the basis just given describes an isometry $\phi: I_{1, l} \rightarrow \mathrm{NS}\left(D_{1}^{-}\right)$. Under $\phi^{-1}$ the exceptional curves of the blow-up $D_{1} \rightarrow \mathbb{P}^{2}$ described in Proposition 6.30 are $\gamma, \gamma+\delta_{l}, \ldots, \gamma+\delta_{l}+\cdots+\delta_{3}$ and $\gamma+\delta_{l}+\cdots+\delta_{3}+\delta_{1}$, while $\delta_{2}$ is the strict transform of a line in $\mathbb{P}^{2}$.

Since $\operatorname{NS}\left(\mathcal{D}_{1}^{-} / \mathcal{Y}\right)$ is constant and $\operatorname{Pic}\left(\mathcal{D}_{1}^{-} / \mathcal{Y}\right) \rightarrow \operatorname{NS}\left(\mathcal{D}_{1}^{-} / \mathcal{Y}\right)$ is an isomorphism, $\phi$ extends to an isometry $\phi: I_{1, l} \rightarrow \operatorname{Pic}\left(\mathcal{D}_{1}^{-} / \mathcal{Y}\right)$. Also, $\mathcal{D}_{1}^{-}$contains an anticanonical divisor $\mathcal{A}$ that is a copy of $\mathcal{E} \times \mathcal{M}_{E l l} \mathcal{Y}$; the isomorphism $\mathcal{A} \rightarrow \mathcal{E} \times{ }_{\mathcal{M}_{E l l}} \mathcal{Y}$ is provided by the base point $\gamma \cap \mathcal{A}$.

Restricting to $\mathcal{A}$ provides a homomorphism

$$
\psi: I_{1, l} \rightarrow \operatorname{Pic}(\mathcal{A} / \mathcal{Y}) \cong \operatorname{Pic}(\mathcal{E})=\coprod_{n \in \mathbb{Z}} \operatorname{Pic}^{n}(\mathcal{E})
$$

Define $\kappa_{l}=\phi^{-1}\left[\omega_{\mathcal{D}_{1} / \mathcal{Y}}^{-1}\right]$, the anti-canonical class. Then

$$
\kappa_{l}=\delta_{1}+\delta_{2}+2 \delta_{3}+3 \sum_{i \geq 4} \delta_{i}+3 \gamma
$$

Note that $I_{1, l} / \mathbb{Z} \kappa_{l}$ is the weight lattice $P$, while $\oplus \mathbb{Z} \delta_{i}$ is the root lattice $Q$. Consider the positive cone $C_{+}$in $I_{1, l} \otimes \mathbb{R}$ defined by the conditions $(v, v) \geq 0, v \cdot \kappa_{l} \geq 0$; this inherits a finite decomposition into chambers from the decomposition of $P \otimes \mathbb{R}$ into fundamental domains for the $W$-action. One such is the chamber $C_{0}$ defined by the inequalities $\left(v, \delta_{i}\right) \geq 0$ for all $i$.

Lemma $\left.7.8 \omega_{\mathcal{D}_{1}^{-} / \mathcal{Y}}^{-1}\right|_{\mathcal{A}}$ is isomorphic to the pullback of $\mathcal{O}_{E}\left(d\left[O_{E}\right]\right)$ to $\mathcal{A}=\mathcal{E} \times \times_{\mathcal{M}_{E l l}}$ $\mathcal{Y}$.

PROOF: $\quad \mathcal{D}_{0}^{-}$is the minimal resolution of a singular normal surface that is defined over the stack $\mathcal{M}_{\text {Ell }}$ of elliptic curves, and so $\mathcal{N}_{\mathcal{A} / \mathcal{D}_{0}^{-}}$is defined over $\mathcal{M}_{\text {Ell }}$. So $\mathcal{N}_{\mathcal{A} / \mathcal{D}_{0}^{-}} \cong \mathcal{O}_{E}\left(-d\left[O_{E}\right]\right)$. Since $\mathcal{N}_{\mathcal{A} / \mathcal{D}_{0}^{-}} \cong \mathcal{N}_{\mathcal{A} / \mathcal{D}_{1}^{-}}^{\vee}$, the result follows from the adjunction formula and the triviality of $\omega_{\mathcal{Z}^{-}} / \mathcal{L}$.

By Lemma 7.8, $\psi\left(\kappa_{l}\right)=d\left[O_{E}\right]$, so there is a homomorphism $\bar{\psi}: P \rightarrow \operatorname{Pic}_{E}^{0}$ defined by $\bar{\psi}(L)=\psi(L)-\operatorname{deg} \psi(L)\left[O_{E}\right]$.

Now consider the stack $\mathcal{M} d \mathcal{P}_{d}$ of marked weak del Pezzo surfaces of degree $d$; by definition, the objects consist of:

(1) a family $f: X \rightarrow S$ of weak del Pezzo surfaces of degree $d$;

(2) an embedding $i: E \times S \hookrightarrow X$ such that the class of the image $\mathcal{A}$ equals the class $\left[\omega_{X / S}^{-1}\right]$ and $i^{*}\left(\omega_{X / S}^{-1}\right)$ is linearly equivalent to $d\left[O_{E}\right]$; 
(3) an isometry $\phi: I_{1, l} \rightarrow \operatorname{Pic}(X / S)$ such that $\left.\phi(\gamma)\right|_{\mathcal{A}}$ is linearly equivalent to $\left[0_{\mathcal{A}}\right]$ and $\phi\left(\delta_{j}\right)$ has degree 0 for all $j$.

In this language, $\left(\mathcal{D}_{1}^{-} \rightarrow \mathcal{Y}, \mathcal{A} \hookrightarrow \mathcal{D}_{1}^{-}, \phi\right)$ is an object of $\mathcal{M} d \mathcal{P}_{d}$, so defines a morphism $H: \mathcal{Y} \rightarrow \mathcal{M} d \mathcal{P}_{d}$.

There is also a morphism $F: \mathcal{M} d \mathcal{P} \rightarrow \mathcal{Y}$ defined by $(f, i, \phi) \mapsto \psi^{\prime}$, where $\psi^{\prime}$ is constructed exactly as above, and a morphism $G: \mathcal{Y} \rightarrow \mathcal{M} d \mathcal{P}$, as follows:

Given $\psi^{\prime}: P \rightarrow E$, construct $\psi: I_{1, l} \rightarrow \operatorname{Pic}(E)$ by

$$
\psi(\gamma)=\left[0_{E}\right], \quad \psi\left(\delta_{i}\right)=\psi^{\prime}\left(\delta_{i} \quad\left(\bmod \mathbb{Z} \kappa_{l}\right)\right) .
$$

Then $\psi\left(\kappa_{l}\right)$ has degree 3. Embed $E \hookrightarrow \mathbb{P}^{2}$ via $\left|\psi\left(\kappa_{l}\right)\right|$ and then make $l$ blow-ups $\mathbb{P}^{2}$ along the points on $E$ defined by the degree 1 classes

$$
\psi\left(\gamma+\delta_{l}+\cdots+\delta_{3}+\delta_{1}\right), \psi\left(\gamma+\delta_{l}+\cdots+\delta_{3}\right), \ldots, \psi\left(\gamma+\delta_{l}\right), \psi(\gamma)
$$

in that order.

The next result is due to Mérindol [M]. Its proof is an immediate consequence of the definitions of $F$ and $G$.

Proposition $7.9 F \circ G=1 \mathcal{Y}$.

Corollary 7.10 $F$ exhibits $\mathcal{Y}$ as the maximal separated quotient of $\mathcal{M} d \mathcal{P}$.

PROOF: Immediate.

That is, $\mathcal{M d} \mathcal{P}$ is obtained by glueing together copies of $\mathcal{Y}$ along open subvarieties. On the locus of del Pezzo surfaces that have no $(-2)$-curves the stacks $\mathcal{M} d \mathcal{P}$ and $\mathcal{Y}$ are isomorphic but $\mathcal{M} d \mathcal{P}$ fails to be separated when $(-2)$ curves appear.

Proposition $7.11 F \circ H=1_{\mathcal{Y}}$.

PROOF: $F \circ H$ takes $0_{\mathcal{Y}}$ to $0_{\mathcal{Y}}$, so is a homomorphism of abelian schemes. Fix a geometric elliptic curve $E$; then over $0_{Y}$ the fibre of $\mathcal{D}_{1}$ contains a $(-2)$ configuration of type $G$. Consider the subdiagram

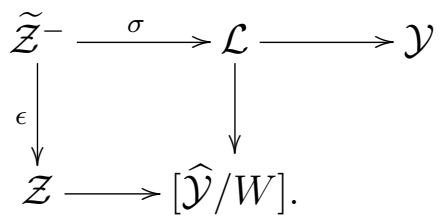

of the diagram in Proposition 7.2. It follows from Theorem 6.24 that $\mathcal{Z} \rightarrow[\widehat{\mathcal{Y}} / W]$, which is a family of affine surfaces, has du Val singularities of type $G$ exactly over $\mathbb{A}^{1}-\{0\}$, where $\mathbb{A}^{1}$ is the line in the cone $[\widehat{\mathcal{Y}} / W]$ that lies over the image of $0_{\mathcal{Y}}$ in $[\widehat{\mathcal{Y}} / W]$. Therefore $0 \mathcal{y}$ is isolated in the fibre $(F \circ H)^{-1}\left(0_{\mathcal{Y}}\right)$ and then $F \circ H$ is an isomorphism. Since $F \circ H$ is $W$-equivariant it is then \pm 1 .

Suppose that $F \circ H=-1$. Put $S=\mathcal{U} \times_{\mathcal{M} d \mathcal{P}, G} \mathcal{Y}$, where $\mathcal{U} \rightarrow \mathcal{M d \mathcal { P }}$ is universal, and consider the $\mathcal{Y}$-isomorphism $\mathcal{D}_{1} \rightarrow S \times \mathcal{Y}, F \circ H \mathcal{Y}$. Since $F \circ H=-1$, 
this takes the ample cone on $\mathcal{D}_{1}$ to the negative of the ample cone on $S$. This is impossible, so $F \circ H=1$.

The modular interpretation of $\mathcal{Y}$ as an open substack of $\mathcal{M} d \mathcal{P}$ is given as follows.

Suppose that $X$ is a weak del Pezzo surface. Define a line in $X$ to be a class $m$ in $\mathrm{NS}(X)$ such that $m^{2}=-1$ and $m \cdot\left(-K_{X}\right)=1$ and a root in $X$ to be a class $\delta$ in $\mathrm{NS}(X)$ such that $\delta^{2}=-2$ and $\delta \cdot K_{X}=0$. Let $\Lambda$ denote the set of lines and $\Delta$ the set of roots on $X$. Then $\Delta$ is a finite root system and reflections in the roots generate a finite Weyl group $W$, as usual. A root $\delta$ is effective if $H^{0}(X, \delta) \neq 0$.

Define the positive cone $C^{+}=C^{+}(X)$ by $C^{+}=\left\{x \in \operatorname{NS}(X)_{\mathbb{R}} \mid x^{2}>\right.$ $\left.0, x .\left(-K_{X}\right)>0\right\}$. It is well known that a class $x$ in $C^{+} \cap \mathrm{NS}(X)$ is nef if and only if $x . m>0$ for all $m \in \Lambda$ and $x . \delta \geq 0$ for all effective roots $\delta$ on $X$. Put $\mathcal{F}=\left\{x \in C^{+} \mid x . m>0 \forall m \in \Lambda\right\}$. Then the roots define walls that tesselate $\mathcal{F}$ into chambers that are permuted simply transitively by $W$. So, if $\mathcal{F}^{\prime}$ is one such chamber, then $\mathcal{F}=\cup_{w \in W} w\left(\mathcal{F}^{\prime}\right)$.

In the lattice $I_{1, l}$, say $\Lambda_{0}=\left\{m \in I_{1, l} \mid m^{2}=-1, m . \kappa=1\right\}, \Delta_{0}=\left\{\delta \in I_{1, l} \mid\right.$ $\left.\delta^{2}=-2, \delta . \kappa=0\right\}, C_{0}^{+}=\left\{x \in I_{1, l} \otimes \mathbb{R} \mid x^{2}>0, x . \kappa>0\right\}$ and $\mathcal{F}_{0}=\left\{x \in C_{0}^{+} \mid\right.$ $\left.x . m>0 \forall m \in \Lambda_{0}\right\}$.

Pick a chamber $\mathcal{F}_{0}^{\prime}$ in the tessellation of $\mathcal{F}_{0}$ defined by the roots in $\Delta_{0}$. Let $\mathcal{M} d \mathcal{P}^{+}$be the open substack of $\mathcal{M} d \mathcal{P}$ whose objects are triples $(X \rightarrow S, i, \phi)$ such that $\phi_{\mathbb{R}}\left(\mathcal{F}_{0}^{\prime}\right)$ is contained in the nef cone of every geometric fibre of $X \rightarrow S$. Then $\mathcal{M} d \mathcal{P}^{+}$is isomorphic to $\mathcal{Y}$, and $\mathcal{M} d \mathcal{P}$ is the union of these copies of $\mathcal{Y}$, one for each chamber $\mathcal{F}_{0}^{\prime}$ in $\mathcal{F}_{0}$.

This brings us to the main result of the paper.

Theorem 7.12 Suppose that $G=E_{l}$ for $l=5,6,7,8$.

(1) The family $\mathcal{Z} \rightarrow[\widehat{\mathcal{Y}} / W]$ has simply elliptic singularities over the vertex of the cone $[\widehat{\mathcal{Y}} / W]$.

(2) Over Spec $\mathbb{Z}[1 /(9-l)]$ this family is a miniversal deformation of these singularities.

(3) The commutative diagram

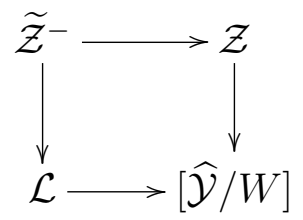

is a simultaneous $\log$ resolution of $\mathcal{Z} \rightarrow[\widehat{\mathcal{Y}} / W]$.

(4) The exceptional divisor $\mathcal{D}_{1}^{-}$in $\widetilde{\mathcal{Z}}^{-}$is the restriction to $\mathcal{Y}$ of the universal marked del Pezzo surface of degree $d=9-l$.

PROOF: (1)-(3) summarize the results of Section 6. (4) follows at once from Corollary 7.10 and the construction of $\widetilde{\mathcal{Z}}^{-}$. 
Remark: Suppose that the base is $\mathbf{S p e c} \mathbb{C}$. The affine del Pezzo surface obtained by deleting the elliptic curve $E$ has a mixed Hodge structure on $H^{2}$. These mixed Hodge structures are naturally parametrized by the Looijenga variety $\mathcal{Y}$. The above theorem shows, when restricted to the exceptional divisor $\mathcal{D}_{1}^{-}$in $\mathcal{Z}^{-}$, that the period map for these affine del Pezzo surfaces is the same as the map from $\mathcal{D}_{1}^{-}$to $\mathcal{Y}$ that we have constructed in terms of group theory.

\section{8 del Pezzo surfaces and the unipotent singu- larity of $E_{8}$ in characteristics 2,3 and 5}

Consider again the family $\widetilde{X}^{-} \rightarrow \mathbb{A}^{1}$ where $\mathbb{A}^{1}$ is the line in the line bundle $L \rightarrow Y$ lying over $0_{Y}$. The closed fibre of $\widetilde{X}_{0}^{-}$is $\widetilde{X}_{0}^{-}=D_{0}^{-}+D_{1}^{-}$and for $t \neq 0$ the fibre $\widetilde{X}_{t}^{-}=\widetilde{X}_{t}$ contains a configuration $\Gamma_{t}$ of $(-2)$-curves of the same combinatorial type as $G$. When $t=0$ this configuration specializes to an isomorphic configuration $\Gamma_{0}$ which lies in $D_{1}^{-}$and is disjoint from the double curve $A^{-}=D_{0}^{-} \cap D_{1}^{-}$. Recall that $A^{-}$is isomorphic to $E$.

Let $D_{1}^{-} \rightarrow D_{1}^{b}$ be the contraction of $\Gamma_{0}$. Then $D_{1}^{b}$ has a singularity of combinatorial type $G$ that does not lie on the curve $A^{b}$ which is the (isomorphic) image of $A^{-}$.

Proposition 8.1 (1) $\widetilde{X}^{-}$is isomorphic to the line bundle $\omega_{D_{1}^{-}}$.

(2) There is a commutative diagram

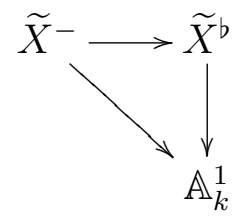

where $\widetilde{X}^{-} \rightarrow \widetilde{X}^{b}$ contracts each $\Gamma_{t}$ to a du Val singularity, $\cup_{t} \Gamma_{t}$ is contracted to a section of $\widetilde{X}^{b} \rightarrow \mathbb{A}_{k}^{1}$ and $\widetilde{X}_{0}^{b}=D_{0}^{b}+D_{1}^{b}$ where $D_{0}^{+} \rightarrow D_{0}^{b}$ is an isomorphism.

(3) $\widetilde{X}^{b}$ is isomorphic to the line bundle $\omega_{D_{1}^{b}}$.

(4) The singular affine surface $\widetilde{X}_{t}$ is isomorphic to the affine del Pezzo surface $D_{1}^{b}-A^{b}$ for all $t \neq 0$.

PROOF: The existence of the contraction $X^{+} \rightarrow X^{b}$ is a special case of the well known fact that a family of $(-2)$-configurations lying in the relative smooth locus of a family of surfaces can be simultaneously contracted. The rest follows from Lemma 6.28.

As has been aready recalled, if $\operatorname{Uni}(G)$ is the unipotent variety of a simply connected simple group $G$ of type $A, D$ or $E$, then the singular locus of $U n i(G)$ is the subregular locus of $U n i(G)$ and the strict localization of $U n i(G)$ at the geometric 
generic point of its subregular locus (we shall refer to this as the unipotent singularity) has a du Val singularity of the same combinatorial type as $G$. However, in low characteristics the combinatorial type does not specify the singularity, even over an algebraically closed field; for example, the $E_{8}$ singularities are classified up to formal isomorphism on p. 270 of [Li] (and up to henselian equivalence in $[\mathrm{Ar}])$. The local equation of each is

$$
t^{2}+z^{3}+y^{5}=\phi
$$

where $\phi \in\left\{0, y^{3} t, y^{3} z t, y^{2} z t, y z t\right\}$ in characteristic $2, \phi \in\left\{0, y^{3} z^{2}, y^{2} z^{2}\right\}$ in characteristic 3 and $\phi \in\left\{0, y^{4} z\right\}$ in characteristic 5. We refer to them as $E_{8}^{(p, \phi)}$ accordingly. In Artin's list [Ar] they are listed instead as $E_{8}^{r}$ where $0 \leq r \leq 4$ or 2 or 1 in these characteristics and $\operatorname{dim} T^{1}=16-2 r$ or $12-2 r$ or $10-2 r$.

For the rest of this section the base field $k$ will be a complete valued algebraically closed field of characteristic $p>0$ and $E$ will be a uniformizable elliptic curve over $k$.

Theorem 8.2 The singularity on $D_{1}^{b}$ is isomorphic to the unipotent singularity of the group $G$.

PROOF: By Theorem 3.11 the singularity of $X_{t \neq 0}$ is isomorphic to the unipotent singularity. The theorem follows from Proposition 8.1.

Recall that in characteristic $p$ the $j$-invariant of a uniformizable elliptic curve $E$ is not algebraic over $\mathbb{F}_{p}$; in particular, it is non-zero.

Until further notice the group $G$ will be $E_{8}$.

Recall that the anticanonical model of a del Pezzo surface $S$ of degree 1 over a field $k$ is a sextic hypersurface in weighted projective space $\mathbb{P}(1,1,2,3)=$ Proj $k\left[X_{1}, Y_{1}, Z_{2}, T_{3}\right]$.

The next result is a counterpart to Proposition 6.30 .

Proposition 8.3 Suppose that $S$ is a del Pezzo surface of degree 1 over a separably closed field $k$ with a singular point $P$ of type $E_{8}$.

(1) Up to projective equivalence, the defining equation of $S$ in $\mathbb{P}(1,1,2,3)$ is

$$
T^{2}+Z^{3}+X Y^{5}=\Phi
$$

where $\Phi \in\left\{Z Y^{4}, 0\right\}$ if char $k \neq 2,3, \Phi \in\left\{Z^{2} Y^{2}, Z Y^{4}, 0\right\}$ if char $k=3$ and $\Phi \in\left\{T Y Z, T Y^{3}, \gamma Z Y^{4}\right\}$ if char $k=2$, where $\gamma=\lambda^{4}+c$, so defines a class in $H_{\text {flat }}^{1}\left(k, \mu_{4}\right)$.

(2) Suppose that $C$ is a smooth member of $\left|-K_{S}\right|$. Then, whatever the characteristic, $j(C)$ is never zero for the first value of $\Phi$ and is always zero for the second. (In characteristics 2 and 3 the pencil $\left|-K_{S}\right|$ is quasi-elliptic for the third value, namely, when $\Phi=0$ or $\gamma Z Y^{4}$.)

(3) In the first case of each characteristic the singularity $(S, P)$ is isomorphic to $E_{8}^{\left(5, y^{4} z\right)}=E_{8}^{1}$ or $E_{8}^{\left(3, y^{2} z^{2}\right)}=E_{8}^{2}$ or $E_{8}^{(2, y z t)}=E_{8}^{4}$. 
PROOF: This depends upon the following recognition principle for $E_{8}$ singularities. It goes back to du Val.

Lemma 8.4 (1) A du Val singularity $(S, P)$ is of combinatorial type $E_{8}$ if and only if the blow-up $\widetilde{S}=\mathrm{Bl}_{P} S$ has a singular point of type $E$.

(2) A du Val singularity whose defining equation can be written as

$$
t^{2}-G(y, z) \in t(y, z, t)^{2}
$$

is of type $E$ if and only if the leading term of $G$ is proportional to a perfect cube.

PROOF: (1) Suppose that $S^{*} \rightarrow S$ is the minimal resolution. Then, according to du Val and Artin, this factors through $\widetilde{S}$ and the fundamental cycle $Z$ on $S^{*}$ is a divisor and the sheaf of ideals that defines it is $\mathfrak{m}_{P} \cdot \mathcal{O}_{S^{*}}$. So, for any component $C_{i}$ of the exceptional locus in $S^{*}, Z . C_{i} \leq 0$, and $Z . C_{i}<0$ if and only if $C_{i}$ appears on $\widetilde{S}$. Moreover, $Z$ is the biggest root; in the case of $E_{8}$ this is the following linear combination of simple roots:

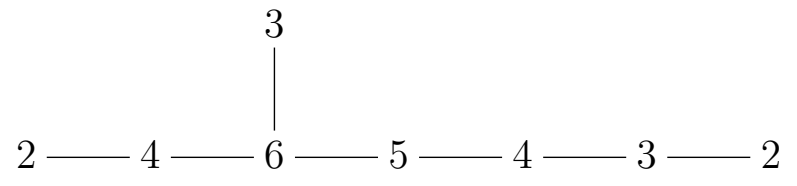

Therefore the curves contracted by $S^{*} \rightarrow \widetilde{S}$ form an $E_{7}$ configuration. Similarly an $E_{7}$ (or $E_{6}$ ) singularity has a $D_{6}$ (or $A_{5}$ ) singularity on its first blow-up.

(2) This is to be found on p. 268 of [Li].

Beyond this lemma the proof of Proposition 8.3 consists of a straightforward manipulation of polynomials and a calculation of $j$-invariants using the standard formulae to be found on, for example, p. 46 of [Si]. We omit the details.

Remark: In particular, over an algebraically closed field of characteristic 2 there are five $E_{8}$ singularities but only three of them can lie on a del Pezzo surface. We do not know what to make of this.

Corollary 8.5 The unipotent singularity of the group $E_{8}$ in characteristics 2,3 and 5 is isomorphic to $E_{8}^{(2, y z t)}=E_{8}^{4}, E_{8}^{\left(3, y^{2} z^{2}\right)}=E_{8}^{2}$ and $E_{8}^{\left(5, y^{4} z\right)}=E_{8}^{1}$, respectively.

PROOF: The unipotent singularity is realized on a del Pezzo surface that contains a uniformizable curve. The $j$-invariant of a uniformizable curve $E$ is transcendental over $\mathbb{F}_{p}$, so that in particular $j(E) \neq 0$, and we can now apply Proposition 8.3 .

Corollary 8.6 The BGSS construction gives a versal deformation of the unipotent singularity of $E_{8}$ in all characteristics.

PROOF: For each du Val singularity Artin computed [Ar] the dimension of $T^{1}$, the tangent space to a miniversal deformation space of the singularity. From his 
list and Corollary 8.5 we can see that $\operatorname{dim} T^{1}=8$ for the unipotent singularity of $E_{8}$. Now the argument of [SB01] goes through unchanged, as it does whenever $\operatorname{dim} T^{1}$ equals the rank of the group.

Corollary 8.7 If $E$ is supersingular, $G=E_{8}$ and char $k=2,3$ or 5 then $\widetilde{\mathcal{G}}^{s s} \rightarrow \mathcal{G}^{s s}$ is not smoothly equivalent to $\widetilde{G} \rightarrow G$ in any neighbourhood of the identity of $G$. That is, the conclusion of Theorem 3.11 fails in these cases.

PROOF: If it were true, then the morphism $\widetilde{\mathcal{G}}^{s s} \rightarrow \mathcal{G}^{s s}$ would be smoothly equivalent to $\widetilde{G} \rightarrow G$ and then the unipotent singularity of $E_{8}$ would be realized on a del Pezzo surface $S$ on which $E$ is an anticanonical curve. Then, according to Proposition 8.3, $S$ can be defined by the equation

$$
T^{2}+Z^{3}+X Y^{5}=\Phi
$$

where $\Phi=0, Z Y^{4}$ or $T Y^{3}$ when char $k=5,3$ or 2 , respectively, since in these characteristics an elliptic curve is supersingular if and only if its $j$-invariant is 0 . But then $S$ has a singularity of type $E_{8}^{(5,0)}$ or $E_{8}^{\left(3, y^{4} z\right)}$ or $E_{8}^{\left(2, y^{3} t\right)}$ and it remains only to check that $E_{8}^{\left(3, y^{4} z\right)}$ is not isomorphic to $E_{8}^{\left(3, y^{2} z^{2}\right)}$. This can be done by calculating $\operatorname{dim} T^{1}$ for $E_{8}^{\left(3, y^{4} z\right)}$; the result is $\operatorname{dim} T^{1}=10$, so that inspection of Artin's list $[\mathrm{Ar}]$ shows that $E_{8}^{\left(3, y^{4} z\right)}$ is in fact isomorphic to $E_{8}^{\left(3, y^{3} z^{2}\right)}$.

For $E_{6}$ and $E_{7}$ similar considerations involving del Pezzo surfaces of degrees 3 and 2 work to describe the unipotent singularity in low characteristics: in the notation of $[\mathrm{Ar}]$ they are $E_{6}^{1}$ and $E_{7}^{3}$ when $p=2$ and $E_{6}^{1}$ and $E_{7}^{1}$ when $p=3$. In all cases the unipotent singularity is the one in its combinatorial class where $\operatorname{dim} T^{1}$ is minimal, so is the "most general" one.

\section{References}

[Ar] M. Artin, Coverings of rational double points in characteristic $p$, in Complex Analysis, W. Baily and T. Shioda, eds.

[AS] J. Armstrong and N. Shepherd-Barron, $A_{g}^{P}$ has terminal singularities for $g \geq 6$, arXiv

[BG] A. Braverman and D. Gaitsgory, Geometric Eisenstein Series, arXiv:math/9912097v2

[BH] H. Bass and W. Haboush, Linearizing certain reductive group actions, Trans. AMS 292 (1985), 463-482.

[BGR] S. Bosch, U. Güntzer and R. Remmert, Non-Archimedean Analysis, Springer, 1984. 
[Br] E. Brieskorn, On the unfolding of exceptional singularities, Nova Acta Leopoldina 52, 1981.

[Brue] G. Bruechert, Trace class elements and cross-sections on Kac-Moody groups, Canad. J. Math. 50 (1998), 972-1006.

[D] D. Davis, thesis, in preparation.

[FM] R. Friedman and J. Morgan, Holomorphic Principal Bundles Over Elliptic Curves II: The Parabolic Construction, J. Diff. Geom. 56 (2000), 301-379.

[GS] I. Grojnowski and N. Shepherd-Barron, Springer fibres revisited, in preparation.

[HS1] S. Helmke and P. Slodowy, Loop groups, elliptic singularities and principal bundles over elliptic curves, Banach Center Publications, Volume 62, 2004.

[HS2] _ On Unstable Principal Bundles over Elliptic Curves, Publ. RIMS, Kyoto Univ. 37 (2001), 349-395

[HS3] Singular elements of affine Kac-Moody groups, Proc. ECM Stockholm (2004).

[Hi] M. Hirokado, Deformations of rational double points and simple elliptic singularities in characteristic p, Osaka J. Math 41 (2004), 605616.

[Li] J. Lipman, Rational singularities with applications to algebraic surfaces and unique factorization, Publ. Math. IHES 36 (1969), 195-279.

[L] E. Looijenga, Homogeneous spaces associated to certain semiuniversal deformations, Proc. ICM (Helsinki, 1978), pp. 529-536.

[L1] $\quad$, Root systems and elliptic curves, Invent. Math. 38 (1976), $17-32$.

[L2] _ Semi-universal deformation of a simple elliptic singularity, II, Topology 17 (1978), 23-40.

[M] J.Y. Mérindol, Les singularités simples elliptiques, leurs déformations, les surfaces de del Pezzo et les transformations quadratiques, Ann. Sci. ENS 15 (1982), 17-44.

[MOSW] R. Muñoz, G. Occhetta, L.E. Solá Conde and K. Watanabe, Rational curves, Dynkin diagrams and Fano manifolds with nef tangent bundle, Math. Ann. 361 (2015), 583-609. 
[P] H. Pinkham, Simple elliptic singularities, Del Pezzo surfaces and Cremona transformations, Proc. Sympos. Pure Math., Vol. XXX, Part 1, 69-71.

[SGA3] Schémas en groupes, Springer Lecture Notes 151-3

[SB01] N.I. Shepherd-Barron, On simple groups and simple singularities, Israel J. Math. 123 (2001), 179-188.

[SB16] An exceptional locus in the perfect compactification of $A_{g}$, arXiv:

[Si] J. Silverman, The arithmetic of elliptic curves, Springer, 1986.

[Sl] P. Slodowy, Simple singularities and simple algebraic groups, Springer Lecture Notes $\mathbf{8 1 5}$.

[SSS] Séminaire sur les singularités des surfaces, Springer Lecture Notes 777.

D.P.M.M.S., Cambridge University, Cambridge CB2 1SB, U.K.

E-mail address: groj@dpmms.cam.ac.uk

King's College London, Strand, London WC2R 2LS, U.K.

E-mail address: Nicholas.Shepherd-Barron@kcl.ac.uk 